\title{
Knots in Electromagnetism
}

\author{
M. Arrayás ${ }^{\mathrm{a},}$, D. Bouwmeester ${ }^{\mathrm{b}, \mathrm{c}}$, J. L. Trueba ${ }^{\mathrm{a}}$ \\ ${ }^{a}$ Área de Electrogmanetismo, Universidad Rey Juan Carlos, Tulipán s/n, 28943 Móstoles, \\ Madrid, Spain. \\ ${ }^{b}$ Department of Physics, University of California, Santa Barbara, California 93106, USA \\ ${ }^{c}$ Huygens-Kamerlingh Onnes Laboratory, Leiden University, P.o. Box 9504, 2300 RA \\ Leiden, The Netherlands
}

\begin{abstract}
Maxwell equations in vacuum allow for solutions with a non-trivial topology in the electric and magnetic field line configurations at any given moment in time. One example is a space filling congruence of electric and magnetic field lines forming circles lying on the surfaces of nested tori. In this example the electric, magnetic and Poynting vector fields are orthogonal everywhere. As time evolves the electric and magnetic fields expand and deform without changing the topology and energy, while the Poynting vector structure remains unchanged while propagating with the speed of light. The topology is characterized by the concept of helicity of the field configuration. Helicity is an important fundamental concept and for massless fields it is a conserved quantity under conformal transformations.

We will review several methods by which linked and knotted electromagnetic (spin-1) fields can be derived. A first method, introduced by A. Rañada, uses the formulation of the Maxwell equations in terms of differential forms combined with the Hopf map from the three-sphere $S^{3}$ to the two-sphere $S^{2}$. A second method is based on spinor and twistor theory developed by R. Penrose in which elementary twistor functions correspond to the family of electromagnetic torus knots. A third method uses the Bateman construction of generating null solutions from complex Euler potentials. And a fourth method uses special conformal transformations, in particular conformal inversion, to generate new linked and knotted field configurations from existing ones. This fourth method
\end{abstract}

Preprint submitted to Physics Reports

November 27, 2016

(C) 2016. This manuscript version is made available under the Elsevier user license http://www.elsevier.com/open-access/userlicense/1.0/ 
is often accompanied by shifting singularities in the field to complex space-time points.

Of course the various methods must be closely related to one another although they have been developed largely independently and they suggest different directions in which to expand the study of topologically non-trivial field configurations. It will be shown how the twistor formulation allows for a direct extension to massless fields of other spin values, such as spin-2 fields satisfying the linearized Einstein vacuum equation, and how the formulation by A. Rañada can be extended to fields for which the electric and magnetic fields are not orthogonal everywhere.

Underlying the various methods is the fact that electric and magnetic field lines can be described as the level curves of complex functions. Compactification of $R^{3}$ naturally leads to finite energy solutions because the fields at infinity in all directions should all converge towards zero. An intriguing question that is raised by the finite energy is whether there is a connection to the quantization of the classical electromagnetic field. We will review some issues related to this question.

Another interesting question is why the general formulation of topologically non-trivial solutions uses the electric and magnetic fields instead of the electromagnetic vector potentials. This leads to a discussion of the Clebsch representation of the electromagnetic field strength 2-form.

Finally, a topic of great interest is the possibility of experimentally generating and investigating linked and knotted field configurations. Since the non-trivial topological field solutions exploit the special conformal symmetry of the underlying vacuum wave-equations it will only be possible to approximate the solutions in an experiment, which necessarily introduces material objects that will break the special conformal symmetry. We will review the research on plasma configurations in which the magnetic field-line configuration approximates plasma torus knots leading to the prediction of topological solitons in plasma. 
Keywords: Electromagnetic knots, topology, helicity, Hopf map, Clebsh representation, twistor, conformal symmetry, plasmas.

\section{Introduction}

Electric and magnetic fields where described by Faraday, Maxwell and others in the XIX century as vector fields that can be visualized using field lines or lines of force. A field line is a geometrical object, a curve in space, such that the 5 tangent line at each of its points is parallel to the vector field at that point. The lines of force, both electric and magnetic, were considered by Faraday to be real physical objects [1]. Faraday's original view was supported during a good part of the XIX century leading to attempts to explain the lines of force in terms of the streamlines and vorticity lines of the aether. It was in this context that in 1867 Kelvin proposed that atoms were knots or links of the vortex lines of the aether, a picture presented expressively in his paper called "On vortex atoms" [2]. This idea lost support in later years especially after the advances of the theory of relativity and quantum mechanics. In some sense string theory is a revival of the idea that elementary particles can be represented by linked and knotted structures.

Topology have been, and still is, crucial in the development of the theory of electromagnetism. Topology concepts are already present even at very elementary stages. Gauss and Stokes' theorems are valid only if the surfaces are orientable. The property of orientability is a topological characteristic. In 1833 Gauss [3] considered two linked circuits and established the relation between the magnetic field induced by the currents along the circuits and a topological invariant known as the linking number. An extension of this concept led to the definition of magnetic helicity. Gauss had been fascinated by knots and in fact it was his student, Listing, who introduced the word "Topology" in the context of the study of knots in 1847.

Topological arguments also played an essential role in Dirac's proposal of the monopole in 1931 [4], which implies a mechanism for the quantization of 
the electric charge. Furthermore, since 1959, when Aharonov and Bohm [5] discovered the effect that bears their name, it is known that the description of certain electromagnetic phenomena does require topological considerations. Another interesting example of topology in physics is the Skyrme model [6]. Other developments from pure mathematics turned out to be related to YangMills field theory, such as Witten's proposal of a topological quantum field theory [7].

In this review article we will be mainly dealing with developments in the application of topological concepts for the construction of linked and knotted electromagnetic (EM) field configurations. This development was largely initiated by two articles by A. F. Rañada on "Topological electromagnetism" in 1989 [8] and on "Knotted solutions of the Maxwell equations in vacuum" in 1990 [9] in which the Hopf map was used to formulate a linked EM field configuration. A precurser of this work was published by A. Trautman in 1977 in an article on "Solutions of the Maxwell and Yang-Mills equations associated with Hopf fibrings" [10]. As often happens in science, closely related earlier works written in a different context (here in the context of plasma physics [11]), or in

45 a different mathematical formalism (here in the twistor formalism $[12,13,14]$ ) have been identified and will also be reviewed in this article.

Rañada considered solutions to the Maxwell equations in vacuum in which the magnetic and electric field lines are defined by the level curves of a pair of complex scalar fields $\phi(\mathbf{r}, t), \theta(\mathbf{r}, t): R^{3} \times R \rightarrow C$ :

$$
\begin{aligned}
& \mathbf{B}(\mathbf{r}, t)=\frac{\sqrt{a}}{2 \pi i} \frac{\nabla \phi \times \nabla \bar{\phi}}{(1+\bar{\phi} \phi)^{2}}=\frac{\sqrt{a}}{2 \pi i c(1+\bar{\theta} \theta)^{2}}\left(\frac{\partial \bar{\theta}}{\partial t} \nabla \theta-\frac{\partial \theta}{\partial t} \nabla \bar{\theta}\right), \\
& \mathbf{E}(\mathbf{r}, t)=\frac{\sqrt{a} c}{2 \pi i} \frac{\nabla \bar{\theta} \times \nabla \theta}{(1+\bar{\theta} \theta)^{2}}=\frac{\sqrt{a}}{2 \pi i(1+\bar{\phi} \phi)^{2}}\left(\frac{\partial \bar{\phi}}{\partial t} \nabla \phi-\frac{\partial \phi}{\partial t} \nabla \bar{\phi}\right),
\end{aligned}
$$

where $\bar{\phi}$ and $\bar{\theta}$ are the complex conjugates of $\phi$ and $\theta$ respectively, $i$ is the imaginary unit, $a$ is a constant introduced so that the magnetic and electric fields have correct dimensions, and $c$ is the speed of light in vacuum.

Since compactified $R^{3}$ ( $R^{3}$ combined with a single point representing infinity in all directions) is isomorphic to the three-sphere $S^{3}$ via stereographic projec- 
tion and since the complex plane combined with a point at infinity is isomorphic to the two-sphere $S^{2}$, there is a natural role for maps from $S^{3}$ to $S^{2}$ in describing the complex scalar fields $\phi(\mathbf{r}, t), \theta(\mathbf{r}, t)$, provided two physical conditions are satisfied associated to the added point at infinity in $R^{3}$ and the added point at infinity in the complex plane. The first condition is that the scalar fields $\phi$ and $\theta$ ${ }_{60}$ in $R^{3}$ converge to a single value when approaching infinity in any direction. The natural choice is to take the value to be zero. This implies that such source-free fields have the desirable physical property of finite energy. The second condition is that field lines closest to the field line associated to the point at infinity in the complex plane must all become identical. This is possible if the field in $R^{3}$ has a central circle to which the nearby field lines degenerate down to. Hopf investigated maps from $S^{3}$ to $S^{2}$ in which closed loops are mapped to points. Different loops on $S^{3}$ can be linked to each other and knotted, leading after stereographic projection to linked and knotted fields in $R^{3}$.

The Hopf-Rañada solution was obtained by the following complex scalar functions in which the Hopf map is implicit [15],

$$
\begin{gathered}
\phi=\frac{(A X-T Z)+i(A Y+T(A-1))}{(A Z+T X)+i(A(A-1)-T Y)} \\
\theta=\frac{(A Y+T(A-1))+i(A Z+T X)}{(A X-T Z)+i(A(A-1)-T Y)}
\end{gathered}
$$

In these expressions, coordinates $(X, Y, Z, T)$ are dimensionless and $A=\left(R^{2}-\right.$ $\left.T^{2}+1\right) / 2$, with $R=X^{2}+Y^{2}+Z^{2}$. Per construction the EM field lines are linked loops filling the surfaces of nested tori. The electric field configuration is everywhere orthogonal to the magnetic field configuration. Two characteristic features, the energy distribution falling off to zero at infinitiy and the presence of a central circle, follow from the compactifications of $R^{3}$ and the complex plane as needed for the application of the Hopf map. Fig. 1 illustrates the field line configuration.

The Hopf-Rañada solution is a null field: the electric and magnetic fields are perpendicular and they have the same modulus in natural units. The topology is conserved during the time evolution, therefore the field lines remain linked 

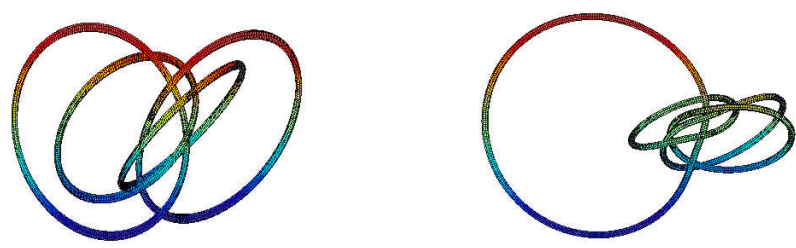

Figure 1: Structure of magnetic lines of the electromagnetic Hopfion. (a) Four magnetic lines at $T=0$. Any pair of them is linked, and the linking number is always equal to 1 . (b) The same situation occurs when $T=0.2$.

and closed. The Gauss linking number for the fields coincides with the Hopf index, a number closely related to helicity which is an important concept in the characterization of fields with non-trivial topology [16].

The initial papers by Rañada and its subsequent refinements inspired the search for possible experimental routes for creating linked and knotted light beams, as well as the search for generalizations of the Hopf-Rañada field [17]. This search led to two new classes of torus knots. One class represents non-null fields in which helicity is exchanged between the electric and magnetic fields [18]. The other class represents null-fields, characterized by the electric and magnetic fields being perpendicular and having the same modulus in natural units [19]. This class was obtained following the Bateman construction for generating EM null fields [20]. Furthermore, methods of deriving topologically non-trivial fields have been explored using conformal transformations of known solutions combined with shifts of space-time parameters into the complex plane $[21,14]$.

Among the applications, the concept of linked and knotted magnetic field lines to magnetohydrodynamics has led to the suggestion that the ball lightning phenomenon could be explained in this way [22]. More recently self-organizing plasma knots have been observed in numerical simulations, where the initial configuration contained helicity in the form of a few linked magnetic field rings [23]. It should be mentioned that the Hopf fibration and torus knots have also 
generated a lot of activities in other research areas such as liquid crystals [24], Bose-Einstein condensates [25, 26] and hydrodynamics [27] but those topics are outside the scope of this review.

The plan of this article is as follows. In Section 2, we review the concept of duality of the Maxwell theory in vacuum, which is important to understand the role played by the Bianchi identities in the derivation of the Hopf-Rañada field. In order to understand the relation between the equations that define an electromagnetic knot and the field lines, we introduce the Clebsh representation of electromagnetic fields in vacuum. Then the helicity for the field lines is defined through the vector potentials. Helicity is a crucial concept for this review article and we have included Appendix A describing the relation between the Gauss integral, the linking number and the helicity. We show how the Euler potentials provide a Clebsh representation of the fields and how they are defined in term of the scalar maps $\phi$ and $\theta$. We present some examples, and show the local equivalence of the Rañada formulation and the solutions of the Maxwell equations in vacuum.

In Section 3 we discuss the general properties of the Rañada construction when the scalar fields are restricted to applications from the three dimensional sphere $S^{3}$ to the two dimensional sphere $S^{2}$. Topological properties of such maps are classified in homotopy classes using the Hopf index.

Section 4 gives a full account of the Hopf-Rañada field which was briefly presented in this introduction. We give the Euler potentials of the so-called Hopfion, and study the properties of the magnetic surfaces which are the points where the Euler potentials take constant values. We will see the fibration of the Hopfion, that is the structure of all the magnetic and electric field lines at a given instant of time. We will see how the energy density spreads out and the motion of electric changes under its influence [28].

Section 5 is devoted to the non-null torus knotted class of electromagnetic fields. They were found as an extension to the Hopf-Rañada solution [18], and in general the members of this class do not have electric and magnetic field perpendicular to each other (note as we will see that the Hopfion is a member 
of this class and the Hopfion is a null field). Within this class, initially all the magnetic lines and electric lines are linked torus knots. Torus knots are knotted curves lying on the surface of a torus.

In Section 6 another class of fields that also has the torus topology for the field lines is presented. However, this class is null, meaning that the electric and magnetic fields are orthogonal. We present the Bateman construction, based on complex Euler potentials in this section.

In Section 7 we provide a very different derivation of the Hopf-Rañada field by applying special conformal transformations to very simple (but unphysical) solutions, such as constant and orthogonal E and B. The conformal transformations form a 15 dimensional group which has the (6 dimensional) proper Lorentz group and the (10 dimensional) Poincaré group as subgroups. The 5 special conformal transformations are composed of a conformal inversion followed by a (4-dimensional) translation followed by another conformal inversion. In order to get a feeling for what special conformal transformations are let's look at conformal inversion in $R^{3}$. Conformal inversion for a vector in $R^{3}$ means $\mathbf{x} \rightarrow \mathbf{x} / \mathbf{x} \cdot \mathbf{x}$. Let us take a plane in $R^{3}$ defined by $x=1$ and a constant electric field in this plane pointing in the $z$ direction. After conformal inversion each field line will turn into a circle that touches the origin and the collection of all those circles will form a sphere that touches the origin and the point $(1,0,0)$. Taking the same initial planes of constant $\mathbf{E}$ field but for different values of $x$ will produce a space filling congruence of 2 sets of nested spheres (for $x<0$ and $x>0$ ). All spheres touch in the origin at which the field strength becomes infinite. If we had also started with an orthogonal constant $\mathbf{B}$ field in each field (equal in magnitude in natural units to the $\mathbf{E}$ field and pointing in the $y$ direction) we would get on each sphere a $\mathbf{B}$ field orthogonal to the $\mathbf{E}$ field and equal in magnitude. If we consider $t=0$ in Minkowski space we will have the same situations as just described, up to a minus sign in the norm which simply flips all field vectors. If we subsequently consider a time translation that consists of a purely imaginary value, followed by another conformal inversion one finds precisely the Hopf-Rañada electric field configuration [29, 21]. This 

inertial reference frame combined with complex shifts is familiar to other disciplines. For example the Kerr solution can be obtained in a similar fashion from the Schwarzschild solution and Gaussian beams can be generated from spherical waves [30].

170 configurations. This method is part of the twistor theory developed by R. Penrose and his colleagues over several decades. The applications of this theory are wide and deep. The original goal of the theory is to unify general relativity and quantum mechanics. Since light rays, following geodesics, define the space

method of applying transformations that cannot be seen as just a change of

In Section 8 we review the twistor formulation of linked and knotted field time metric it would perhaps be advantageous to formulate the laws of physics in a space, the twistor space, in which each point, a twistor, represents a light ray (or a ray of another massless spin $n / 2$ field). The norm of a twistor is its helicity, which is invariant under the full conformal group. Solutions to the wave equations can be derived from twistor functions of specific homogeneity followed by the Penrose transform. In Appendix B we summarise the bare minimum of the basics of twistor theory needed to understand the construction of the linked and knotted massless field configurations. One of the strengths of the twistor approach is that solutions to all massless wave equations are derived in the same manner. Therefore we can directly identify linked and knotted solutions for the spin 2 linearized Einstein equations in vacuum.

In Section 9 we make a connection between EM knots and plasma physics. Since plasma is highly conductive, internal electric fields are generally small while magnetic fields can be large and can strongly determine the dynamics through the resulting Lorentz forces on moving charges. The equations of magnetohydrodynamics (MHD) describe the interplay between the magnetic forces resulting from currents and pressure gradient forces. Kamchatnov showed how a skeleton of a magnetic Hopfion (the magnetic part of the Hopf-Rañada field at $t=0)$ can result in a quasi-stable plasma configuration [11]. The configuration is neither an equilibrium state nor a lowest energy ground state, however it is 195 still robust against decay because conservation of energy and angular momen- 
tum combined with the topological invariance of magnetic helicity (in an ideal dissipationless plasma) prevents the configuration from expanding and radiating away its energy. This so-called topological soliton in plasma can be generalized to torus knot magnetic skeletons but only if the poloidal winding number of equations in vacuum possesses and uses the language of tensors and differential forms in the Minkowski space-time. We first review briefly those concepts. Then we will present the construction and discuss some of the properties of this formulation in terms of Clebsch variables. We will see the connection between as the helicity concept is related to the topology of the fields. Electromagnetic fields in vacuum can be expressed in terms of Euler potentials which are useful to describe the topology of field lines. We will show that the fields defined in terms of the scalars introduced by Rañada are locally equivalent to the class of 


\subsection{Maxwell theory in vacuum}

Here we summarise some known facts of Maxwell theory of electromagnetism in vacuum making use of tensors and differential forms in a space-time with a Minkowski metric. magnetism in vacuum. Using the SI of units, Maxwell's equations in vacuum are written as

$$
\begin{array}{ll}
\nabla \cdot \mathbf{B}=0, & \nabla \times \mathbf{E}+\frac{\partial \mathbf{B}}{\partial t}=0 \\
\nabla \cdot \mathbf{E}=0, & \nabla \times \mathbf{B}-\frac{1}{c^{2}} \frac{\partial \mathbf{E}}{\partial t}=0
\end{array}
$$

where $\mathbf{E}$ and $\mathbf{B}$ are the electric and magnetic vector fields. The first two equations are identities when the electromagnetic potentials $V, \mathbf{A}$ are introduced. We will take Greek indices $\mu, \nu, \ldots=0,1,2,3$, for four-dimensional quantities in Minkowski space-time, while Latin indices $i, j, \ldots=1,2,3$, will be three-dimensional space components. Unless otherwise stated, we will take the Minkowski metric as $\eta_{\mu \nu}=\eta^{\mu \nu}=<1,-1,-1,-1>$, where the notation $\langle a, b, \ldots\rangle$ means a diagonal matrix with elements $a, b, \ldots$. We will follow

Einstein summation notation, so repeated indices are implicitly summed over.

The four-vector electromagnetic potential has components

$$
A^{\mu}=\left(\frac{V}{c}, \mathbf{A}\right),
$$

where $V$ and $\mathbf{A}$ are the usual scalar potential and vector potential, respectively, and the electromagnetic field tensor is defined by

$$
F_{\mu \nu}=\partial_{\mu} A_{\nu}-\partial_{\nu} A_{\mu}
$$

We will use the notation $\partial_{\mu} f=\partial f / \partial x^{\mu}$, so that $\partial_{0} f=(1 / c) \partial f / \partial t, \partial_{1} f=$ $\partial f / \partial x$, etc. From the electromagnetic field tensor (6), the electric and magnetic field components are $\mathbf{E}_{i}=c F^{i 0}$ and $\mathbf{B}_{i}=-\epsilon_{i j k} F^{j k} / 2$, being $\epsilon_{i j k}=\epsilon^{0 i j k}$ and 
$\epsilon^{\mu \nu \alpha \beta}$ the totally antisymmetric tensor with $\epsilon^{0123}=1$. In three-dimensional quantities, equation (6) is written as the pair of equations

$$
\mathbf{E}=-\nabla V-\frac{\partial \mathbf{A}}{\partial t}, \quad \mathbf{B}=\nabla \times \mathbf{A} .
$$

Using the language of differential forms (a good introduction to differential forms and their applications to electromagnetism can be found in [42]), we can write the four-vector potential as the 1-form $A=A_{\mu} d x^{\mu}$, and the electromagnetic field by the 2 -form

$$
F=\frac{1}{2} F_{\mu \nu} d x^{\mu} \wedge d x^{\nu}
$$

where $\wedge$ denotes the exterior product between forms. The exterior derivative of the 1-form $A$ satisfies $d A=F$. Now, given the property $d^{2}=0$ of the exterior derivative,

$$
d F=d^{2} A=0,
$$

or in tensor components,

$$
\epsilon^{\mu \nu \alpha \beta} \partial_{\nu} F_{\alpha \beta}=0
$$

In three-dimensional form, equation (10) is written as the pair of equations (3). Consequently, these equations are purely geometrical identities, without dynamical content. Equation (9) is known as a Bianchi identity (for its geometrical meaning see [43]).

The dynamics of the electromagnetic field in vacuum is given by the second pair of Maxwell equations (4), that can be written as the tensor equation

$$
\partial_{\mu} F^{\mu \nu}=0
$$

or in the language of differential forms as

$$
d^{*} F=0,
$$

where ${ }^{*} F$ is the dual of the form (8),

$$
{ }^{*} F=\frac{1}{4} \epsilon_{\mu \nu \alpha \beta} F^{\mu \nu} d x^{\alpha} \wedge d x^{\beta} .
$$


We can write ${ }^{*} F$ as

$$
{ }^{*} F=\frac{1}{2}{ }^{*} F_{\mu \nu} d x^{\mu} \wedge d x^{\nu},
$$

where the dual of the electromagnetic field $F_{\mu \nu}$ is defined as

$$
{ }^{*} F_{\mu \nu}=\frac{1}{2} \epsilon_{\mu \nu \alpha \beta} F^{\alpha \beta} .
$$

${ }_{245}$ The components of the tensor ${ }^{*} F_{\mu \nu}$ are given by $\mathbf{B}_{i}={ }^{*} F^{i 0}, \mathbf{E}_{i}=c \epsilon_{i j k}{ }^{*} F^{j k} / 2$. Thus electromagnetism in vacuum is given by a closed two-form $F=d A$ in the Minkowski space-time, constrained by the dynamical equation $d^{*} F=0$.

Let us examine the meaning of the duality property. Maxwell equations in vacuum are invariant under the replacement of the pair of fields $(\mathbf{E}, c \mathbf{B})$ by the pair $(c \mathbf{B},-\mathbf{E})$. The dynamical equation $d^{*} F=0$ implies that ${ }^{*} F$ is a closed two-form, so it is also exact in the Minkowski space-time. This means that we can write

$$
{ }^{*} F=\frac{d C}{c},
$$

where $C$ is a one-form and the factor $1 / c$ appears because of dimensional requirements. The dynamical equation $d^{*} F=0$ becomes $d^{2} C=0$, so it is again a Bianchi identity. Note that the dual of the form ${ }^{*} F$ is ${ }^{* *} F=-F$. Consequently, from the dual point of view, the dynamical equation $d^{*} F=0$ is a Bianchi identity, and the Bianchi identity $d F=0$ is a dynamical equation [44].

As long as we are studying fields in vacuum we can define another fourpotential

$$
C^{\mu}=\left(c V^{\prime}, \mathbf{C}\right)
$$

so that, in tensor components,

$$
{ }^{*} F_{\mu \nu}=-\frac{1}{c}\left(\partial_{\mu} C_{\nu}-\partial_{\nu} C_{\mu}\right),
$$

or, in three-dimensional components,

$$
\mathbf{E}=\nabla \times \mathbf{C}, \mathbf{B}=\nabla V^{\prime}+\frac{1}{c^{2}} \frac{\partial \mathbf{C}}{\partial t} .
$$

The identity $d^{*} F=0$, expressed in function of $\mathbf{E}$ and $\mathbf{B}$, is the pair of equations (4), and the dynamical equation $d F=0$ is the pair of equations (3). 
Exact solutions of Maxwell equations (3)-(4) in which magnetic and electric field lines coincide with level curves of a pair of complex scalar fields $\phi(\mathbf{r}, t)$, $\theta(\mathbf{r}, t): R^{3} \times R \rightarrow C$ can be written as functions of such scalars.

Let us discuss what can be deduced about the actual fields from the evolution of their magnetic and electric lines. We start by representing the magnetic lines by the equation $\phi(t, x, y, z)=\phi_{0}$ where $\phi$ is a complex function of space and time and $\phi_{0}$ is a constant labelling each line. This means that the magnetic lines are the level curves of the complex scalar field $\phi(t, x, y, z)$. Since the magnetic field is tangent to the magnetic lines at each point, it has to satisfy

$$
\mathbf{B}(\mathbf{r}, t)=g(\mathbf{r}, t) \nabla \phi \times \nabla \bar{\phi} .
$$

The magnetic field has zero divergence. Applying that property to the expression (20), one gets

$$
\nabla \cdot \mathbf{B}=\nabla g \cdot(\nabla \phi \times \nabla \bar{\phi})=0 .
$$

For this equation to be fulfilled, it is necessary that the function $g$ depends on coordinates $(\mathbf{r}, t)$ only through $\phi$ and $\bar{\phi}$. Consequently, we get

$$
\mathbf{B}(\mathbf{r}, t)=g(\phi, \bar{\phi}) \nabla \phi \times \nabla \bar{\phi}
$$

The magnetic field can also be written as $\mathbf{B}_{i}=-\epsilon_{i j k} F^{j k} / 2$ if we take

$$
F_{i j}=g(\phi, \bar{\phi})\left(\partial_{i} \bar{\phi} \partial_{j} \phi-\partial_{j} \bar{\phi} \partial_{i} \phi\right)
$$

Covariance implies that the electromagnetic tensor can be defined as

$$
F_{\mu \nu}=g(\phi, \bar{\phi})\left(\partial_{\mu} \bar{\phi} \partial_{\nu} \phi-\partial_{\nu} \bar{\phi} \partial_{\mu} \phi\right)
$$

The electric field corresponding to (24) is

$$
\mathbf{E}(\mathbf{r}, t)=g(\phi, \bar{\phi})\left(\partial_{0} \bar{\phi} \nabla \phi-\partial_{0} \phi \nabla \bar{\phi}\right) .
$$

The electromagnetic field defined by the complex scalar field $\phi$ that gives the magnetic lines is then such that the electric and the magnetic fields are orthogonal. 
If we consider electromagnetism in vacuum, there is a duality between the magnetic and electric parts of the electromagnetic field, so we can study the electric lines as level curves of another complex scalar field $\theta$. The dual expressions to (20)-(25) are the following. The electric field is

$$
\mathbf{E}(\mathbf{r}, t)=g^{\prime}(\theta, \bar{\theta}) \nabla \bar{\theta} \times \nabla \theta,
$$

and can be obtained from a dual electromagnetic tensor ${ }^{*} F_{\mu \nu}$ by making the identification $\mathbf{E}_{i}=c \epsilon_{i j k}{ }^{*} F^{j k} / 2$, where

$$
{ }^{*} F_{\mu \nu}=g^{\prime}(\theta, \bar{\theta})\left(\partial_{\mu} \bar{\theta} \partial_{\nu} \theta-\partial_{\nu} \bar{\theta} \partial_{\mu} \theta\right) .
$$

The magnetic field is then

$$
\mathbf{B}(\mathbf{r}, t)=\frac{g^{\prime}(\theta, \bar{\theta})}{c}\left(\partial_{0} \bar{\theta} \nabla \theta-\partial_{0} \theta \nabla \bar{\theta}\right)
$$

To obtain a solution of Maxwell equations in vacuum, expressions (22) and (28) have to be equal for the magnetic field, and expressions (25) and (26) have to be equal for the electric field. This duality condition must be fulfilled.

A possible choice for the functions $g$ and $g^{\prime}$ is [8]

$$
\begin{aligned}
g(\phi, \bar{\phi}) & =\frac{\sqrt{a}}{2 \pi i(1+\bar{\phi} \phi)^{2}} \\
g^{\prime}(\theta, \bar{\theta}) & =\frac{\sqrt{a} c}{2 \pi i(1+\bar{\theta} \theta)^{2}},
\end{aligned}
$$

where $a$ and $c$ are constants. The particular form of the dependence of $g$ and $g^{\prime}$ with the complex fields $\phi$ and $\theta$ will become clear later when we consider the compactification of the physical space $R^{3}$ and the complex plane $C$. These compactifications will reveal some aspects of the rich topological structure hidden under the linear Maxwell equations. Before introducing topological tools, we must define these particular fields and study their properties as solutions of Maxwell equations in vacuum.

If $\phi(\mathbf{r}, t)$ and $\theta(\mathbf{r}, t)$ are two complex scalar fields (we will consider them dimensionless), a magnetic field $\mathbf{B}$ and an electric field $\mathbf{E}$ can be defined from 
them as

$$
\begin{aligned}
& \mathbf{B}(\mathbf{r}, t)=\frac{\sqrt{a}}{2 \pi i} \frac{\nabla \phi \times \nabla \bar{\phi}}{(1+\bar{\phi} \phi)^{2}}, \\
& \mathbf{E}(\mathbf{r}, t)=\frac{\sqrt{a} c}{2 \pi i} \frac{\nabla \bar{\theta} \times \nabla \theta}{(1+\bar{\theta} \theta)^{2}},
\end{aligned}
$$

where $\bar{\phi}$ and $\bar{\theta}$ are the complex conjugates of $\phi$ and $\theta$ respectively, $i$ is the imaginary unit, $a$ is a constant introduced so that the magnetic and electric fields have correct dimensions, and $c$ is the speed of light in vacuum. In the SI of units, a can be expressed as a pure number times the Planck constant $\hbar$ times the speed of light $c$ times the vacuum permeability $\mu_{0}$. In natural units, with $\hbar=c=\mu_{0}=\varepsilon_{0}=1, a$ is simply a real number.

In order to obtain a solution of the Maxwell equations in vacuum from the fields given by Equations (30), they also have to satisfy

$$
\begin{aligned}
& \mathbf{B}(\mathbf{r}, t)=\frac{\sqrt{a}}{2 \pi i c(1+\bar{\theta} \theta)^{2}}\left(\frac{\partial \bar{\theta}}{\partial t} \nabla \theta-\frac{\partial \theta}{\partial t} \nabla \bar{\theta}\right), \\
& \mathbf{E}(\mathbf{r}, t)=\frac{\sqrt{a}}{2 \pi i(1+\bar{\phi} \phi)^{2}}\left(\frac{\partial \bar{\phi}}{\partial t} \nabla \phi-\frac{\partial \phi}{\partial t} \nabla \bar{\phi}\right) .
\end{aligned}
$$

The magnetic and the electric fields resulting from (30) and (31) satisfy exactly

Maxwell equations in vacuum as it can be easily checked.

It is possible to write equations (30) and (31) in a covariant way. The electromagnetic tensor $F_{\mu \nu}$ satisfies

$$
F_{\mu \nu}=\frac{\sqrt{a}}{2 \pi i} \frac{\partial_{\mu} \bar{\phi} \partial_{\nu} \phi-\partial_{\mu} \phi \partial_{\nu} \bar{\phi}}{(1+\bar{\phi} \phi)^{2}}
$$

from which the usual relations $\mathbf{B}_{i}=-\epsilon_{i j k} F^{j k} / 2, \mathbf{E}_{i}=c F^{i 0}$ give the first equation in (30) and the second equation in (31). Another antisymmetric tensor $G_{\mu \nu}$ is defined so that

$$
G_{\mu \nu}=\frac{\sqrt{a}}{2 \pi i} \frac{\partial_{\mu} \theta \partial_{\nu} \bar{\theta}-\partial_{\mu} \bar{\theta} \partial_{\nu} \theta}{(1+\bar{\theta} \theta)^{2}}
$$

It is necessary that $G_{\mu \nu}$ and $F_{\mu \nu}$ are dual so that they define an electromagnetic field in vacuum. The condition

$$
G_{\mu \nu}={ }^{*} F_{\mu \nu}=\frac{1}{2} \epsilon_{\mu \nu \alpha \beta} F^{\alpha \beta}
$$


is to be satisfied by the complex scalar fields $\phi$ and $\theta$. Relations $\mathbf{B}_{i}=G^{i 0}$, $\mathbf{E}_{i}=c \epsilon_{i j k} G^{j k} / 2$ give the second equation in (30) and the first equation in (31). The duality condition can also be written as an equality of equations (30) and $(31)$,

$$
\begin{aligned}
& \frac{\nabla \phi \times \nabla \bar{\phi}}{(1+\bar{\phi} \phi)^{2}}=\frac{1}{c(1+\bar{\theta} \theta)^{2}}\left(\frac{\partial \bar{\theta}}{\partial t} \nabla \theta-\frac{\partial \theta}{\partial t} \nabla \bar{\theta}\right), \\
& \frac{\nabla \bar{\theta} \times \nabla \theta}{(1+\bar{\theta} \theta)^{2}}=\frac{1}{c(1+\bar{\phi} \phi)^{2}}\left(\frac{\partial \bar{\phi}}{\partial t} \nabla \phi-\frac{\partial \phi}{\partial t} \nabla \bar{\phi}\right),
\end{aligned}
$$

290 
that define Rañada's construction, we need to introduce canonical variables of an electromagnetic field in vacuum.

We begin by writing the electromagnetic 2 -form $F$ of an electromagnetic field in vacuum as

$$
F=\frac{1}{2} F_{\mu \nu} d x^{\mu} \wedge d x^{\nu}=\frac{\mathbf{E}_{i}}{c} d x^{0} \wedge d x^{i}-\varepsilon_{i j k} \mathbf{B}_{i} d x^{j} \wedge d x^{k},
$$

where $x^{0}=c t, x^{1}=x, x^{2}=y, x^{3}=z$ are coordinates in the Minkowski space-time, and $\mathbf{E}_{i}, \mathbf{B}_{i}$ are the components of the electric and magnetic fields respectively. As long as we are considering fields in vacuum, we can also write the dual to the electromagnetic 2 -form, that is given by

$$
{ }^{*} F=\frac{1}{2}{ }^{*} F_{\mu \nu} d x^{\mu} \wedge d x^{\nu}=\mathbf{B}_{i} d x^{0} \wedge d x^{i}+\varepsilon_{i j k} \frac{\mathbf{E}_{i}}{c} d x^{j} \wedge d x^{k} .
$$

The representation given by equations (36)-(37) is not the simplest possible. Following [46], if $\omega$ is a 2 -form in a 4 -dimensional vector space, given in terms of a basis of 1 -forms $f^{a}(a=1, \ldots, 4)$ by

$$
\omega=\frac{1}{2} \omega_{a b} f^{a} \wedge f^{b},
$$

then there is another basis of 1 -forms $g^{a}(a=1, \ldots, 4)$ such that $\omega$ can be written as

$$
\omega=g^{1} \wedge g^{2}+g^{3} \wedge g^{4}
$$

To see how this works for an electromagnetic field in vacuum, since $F_{\mu \nu}$ is an antisymmetric tensor we can take from (36),

$F=\left(\frac{\mathbf{E}_{1}}{c} d x^{0}+\mathbf{B}_{3} d x^{2}-\mathbf{B}_{2} d x^{3}\right) \wedge\left(d x^{1}+\frac{\mathbf{E}_{2}}{\mathbf{E}_{1}} d x^{2}+\frac{\mathbf{E}_{3}}{\mathbf{E}_{1}} d x^{3}\right)-\left(\frac{\mathbf{E} \cdot \mathbf{B}}{\mathbf{E}_{1}}\right) d x^{2} \wedge d x^{3}$.

Now take the basis of 1 -forms

$$
\begin{array}{r}
e^{0}=\frac{\mathbf{E}_{1}}{c} d x^{0}+\mathbf{B}_{3} d x^{2}-\mathbf{B}_{2} d x^{3}, \\
e^{1}=d x^{1}+\frac{\mathbf{E}_{2}}{\mathbf{E}_{1}} d x^{2}+\frac{\mathbf{E}_{3}}{\mathbf{E}_{1}} d x^{3}, \\
e^{2}=-\left(\frac{\mathbf{E} \cdot \mathbf{B}}{\mathbf{E}_{1}}\right) d x^{2}, \\
e^{3}=d x^{3} .
\end{array}
$$


This basis can be defined except in the points of space-time in which $\mathbf{E}_{1}=0$ (in these points, we can define other basis similar to (41)). With this basis, $F$ can be written as

$$
F=e^{0} \wedge e^{1}+e^{2} \wedge e^{3}
$$

Moreover, if the determinant of the matrix $F_{\mu \nu}$ is zero, then $F$ is even simpler than (42), and there is a pair of 1 -forms $(f, g)$ such that

$$
F=f \wedge g
$$

In this case, the 2-form $F$ has only one term, and can be called decomposable. There are two Lorentz invariants of the electromagnetic field, given by

$$
\begin{array}{r}
-\frac{1}{4} F_{\mu \nu} F^{\mu \nu}=\frac{\mathbf{E}^{2}-c^{2} \mathbf{B}^{2}}{2 c^{2}}, \\
-\frac{1}{4} F_{\mu \nu}{ }^{*} F^{\mu \nu}=\frac{\mathbf{E} \cdot \mathbf{B}}{c} .
\end{array}
$$

The value of (45) determines the explicit way in which we can give the electromagnetic 2 -form. If the value of $(45) \neq 0$, then the 2 -form $F$ is not decomposable and can be expressed as in (42). If the value is equal to 0 , then $\mathbf{E} \cdot \mathbf{B}=0$, so that $e^{2}=0$ in (41). In this case, $F$ is decomposable (the determinant of the matrix $F_{\mu \nu}$ is equal to zero if $\mathbf{E} \cdot \mathbf{B}=0$ ) and can be written as in (43).

The local canonical representation of every electromagnetic field in vacuum can be mathematically described by means of a theorem due to Darboux [47]. Let $\omega$ be a $k$-form defined on a $m$-dimensional differentiable manifold $M$. The rank of $\omega$ is the minimum number $r$ of linearly independent 1-forms such that $F$ can be written as exterior products of them. Clearly $k \leq r \leq m$. Let us consider the case $k=2$, so $\omega$ is an antisymmetric 2 -form on a $m$-dimensional vector space $M$. The Darboux theorem stablish that $\omega$ may have rank $r=m$ only if $m$ is pair, so that $m=2 n$. If $\omega$ has rank $r=m=2 n$ then, in a convenient basis of the vector space $M$, we can write the matrix $\omega$ as

$$
\omega=\left(\begin{array}{cc}
0 & -I \\
I & 0
\end{array}\right)
$$


where $I$ is the $n \times n$ unit matrix. Consequently, if $\omega$ is a symplectic 2 -form (that is, it is closed so that $d \omega=0$ and it has rank $r=2 n$ ) on a smooth manifold $M$, then for every point $x_{0} \in M$ there is a local system of coordinates around $x_{0}$ in which the coefficients $\omega_{i j}$ are constant. This means that there is a local system of coordinates $\left(q^{1}, \ldots, q^{n}, p^{1}, \ldots, p^{n}\right)$ such that

$$
\omega=d q^{i} \wedge d p^{i}
$$

The coordinates $\left(q^{1}, \ldots, q^{n}, p^{1}, \ldots, p^{n}\right)$ in $(47)$ are called canonical coordinates or canonical variables of $\omega$ and they are not unique in general: there are canonical transformations of these coordinates that leave $\omega$ invariant.

For the electromagnetism in vacuum, the electromagnetic 2-form $F$ has rank 4 in general, so that at least four 1-forms are needed to write it in the Minkowski space-time, as we have seen in equation (42). Moreover, $F$ is a closed form due to the Bianchi identity $d F=0$. In conclusion, the electromagnetic form $F$ is symplectic. Then, due to the Darboux theorem it can be written locally as

$$
F=d q^{1} \wedge d p^{1}+d q^{2} \wedge d p^{2} .
$$

The functions $\left(q^{1}, q^{2}, p^{1}, p^{2}\right)$ are canonical coordinates of $F$ and are defined except canonical transformations. When $F$ is written as in (48), it is said that a Clebsch representation is being used [48]. In vacuum, the dual 2 -form ${ }^{*} F$ is also symplectic, as can be seen by comparing equations (36) and (37), which means it can also be expressed in a Clebsch representation as

$$
{ }^{*} F=d v^{1} \wedge d u^{1}+d v^{2} \wedge d u^{2}
$$

The two sets of functions $\left(q^{1}, q^{2}, p^{1}, p^{2}\right)$ and $\left(v^{1}, v^{2}, u^{1}, u^{2}\right)$ are not independent, since they have to satisfy the duality condition

$$
d v^{1} \wedge d u^{1}+d v^{2} \wedge d u^{2}={ }^{*}\left(d q^{1} \wedge d p^{1}+d q^{2} \wedge d p^{2}\right)
$$

As we have seen above, if $\mathbf{E} \cdot \mathbf{B}=0, F$ is decomposable (and so is ${ }^{*} F$ ). In this case, the electromagnetic 2 -forms $F$ and ${ }^{*} F$ have rank 2 . Thus a decomposable 
electromagnetic field can be written locally in terms of canonical variables $(q, p)$ and $(v, u)$ as

$$
\begin{gathered}
F=d q \wedge d p, \\
{ }^{*} F=d v \wedge d u,
\end{gathered}
$$

subjected to a duality condition $d v \wedge d u={ }^{*}(d q \wedge d p)$. Equations (51) also mean that the 2-form of a non-decomposable electromagnetic field in vacuum is locally the sum of two 2 -forms of decomposable electromagnetic fields.

Suppose that a decomposable electromagnetic field is written globally as in equation (51). Then the same functions $(q, p)$ and $(v, u)$ can be used to write the electric and the magnetic fields in all the points of space-time,

$$
\begin{gathered}
\mathbf{B}=\nabla p \times \nabla q=\left(\frac{\partial v}{\partial t} \nabla u-\frac{\partial u}{\partial t} \nabla v\right), \\
\mathbf{E}=c \nabla v \times \nabla u=c\left(\frac{\partial q}{\partial t} \nabla p-\frac{\partial p}{\partial t} \nabla q\right) .
\end{gathered}
$$

\subsection{Vector potentials and helicities}

It can be shown that, if a decomposable electromagnetic field can be written globally as $F=d q \wedge d p$ and the functions $q$ and $p$ are single-valued and welldefined in all the points of space, then the magnetic helicity has to be equal to zero $[49,34,50]$. The same happens with the electric helicity if the global relation ${ }^{*} F=d v \wedge d u$ and the functions $v$ and $u$ are single-valued and well-defined in all the points of space.

Let us introduce the concept of helicity. This is an important quantity since the value of the helicity provides a measure of the topology associated with the field lines. The magnetic helicity is defined as

$$
h_{m}=\frac{1}{2 c \mu_{0}} \int_{D} \mathbf{A} \cdot \mathbf{B} d^{3} r
$$

where $D$ is the spatial part of the 4-four space, which is a 3-dimensional manifold or volume. We will assume that its frontier $\partial D$ is an orientable surface. We recall that $\mathbf{A}$ is defined such as $\mathbf{B}=\nabla \times \mathbf{A}$. The prefactor involving the speed of light $c$ and the vacuum permeability $\mu_{0}$ is introduced in order to have angular 
momentum units for the helicity as done in particle physics. The definition of the magnetic helicity density $\mathbf{A} \cdot \mathbf{B}$ captures the idea of the rotation of the field A along itself. As the curl of a vector measures its rotation around a point, the integrand in (53) gives how much $\mathbf{A}$ rotates around itself times its own modulus. The name of helicity was coined by Moffatt in the context of fluid dynamics [51]. The magnetic helicity provides a measure of the mean value of the linking number of the magnetic field lines (see Appendix A).

Since the electric field in vacuum is also a field with zero divergence, we can use the concept of duality to define the electric helicity of an electromagnetic field in vacuum as

$$
h_{e}=\frac{\varepsilon_{0}}{2 c} \int d^{3} r \mathbf{C} \cdot \mathbf{E}=\frac{1}{2 c^{3} \mu_{0}} \int d^{3} r \mathbf{C} \cdot \mathbf{E},
$$

where $\mathbf{E}=\nabla \times \mathbf{C}$. The electric helicity in (54) has also dimensions of angular momentum and $\varepsilon_{0}=1 /\left(c^{2} \mu_{0}\right)$ is the electric permittivity. By analogy with the magnetic helicity, the electric helicity is also related to the linking number of the electric lines.

Let us consider the case of the magnetic helicity (the case of electric helicity is completely equivalent). If $F=d q \wedge d p$ globally, then the magnetic field can be written as $\mathbf{B}=\nabla p \times \nabla q$. If, moreover, the functions $p$ and $q$ are single-valued, a well-defined vector potential A could be taken so that

$$
\mathbf{A}=\lambda p \nabla q-(1-\lambda) q \nabla p
$$

where $\lambda$ is a real number such that $0 \leq \lambda \leq 1$. Using (52) and (55), it is easy to see that $\mathbf{A} \cdot \mathbf{B}=0$, so the magnetic helicity is zero.

Suppose now that any of the two vector fields $\nabla p$ or $\nabla q$ in (52), for example $\nabla q$, diverges in certain set of points of the space $R^{3}$, so the function $q$ is not globally well-defined. Then, a well-defined vector potential can not be expressed as in (55). Instead, we can take

$$
\mathbf{A}=p \nabla q+\nabla \chi
$$

where the term $\nabla \chi$ is added to correct the possible ill-definition of $p \nabla q$ (note that $\nabla \chi$ may be not globally well-defined either). The magnetic helicity is now 


$$
h_{m}=\frac{1}{2 c \mu_{0}} \int d^{3} r \nabla \chi \cdot(\nabla p \times \nabla q) .
$$

If all the terms in equation (57) were well-defined in all the points of the space equation

$$
\begin{aligned}
\frac{d \mathbf{r}(\tau)}{d \tau} & =\mathbf{B}\left(\mathbf{r}(\tau), t_{0}\right), \\
\left.\mathbf{r}(\tau)\right|_{\tau=0} & =\mathbf{r}_{0} .
\end{aligned}
$$

Here, $\tau$ is the natural parameter of the curve, related to the differential arc length of the curve $d s=\sqrt{(d x)^{2}+(d y)^{2}+(d z)^{2}}$ by the equation $d \tau=d s / B$. The magnetic lines may lie on surfaces or cover three-dimensional domains. The Euler potentials $[52,53]$ of the magnetic field are two real functions $\alpha_{1}(\mathbf{r}, t) \in R$ and $\alpha_{2}(\mathbf{r}, t) \in R$ such that

$$
\mathbf{B}=\nabla \alpha_{1} \times \nabla \alpha_{2} .
$$

By comparing these equation with (52), we can see that the Euler potentials are canonical variables of the magnetic field and that they provide a Clebsch representation of this field. The points where the Euler potentials take a constant value determine a magnetic surface. 
In vacuum, since the electric field also satisfies the condition $\nabla \cdot \mathbf{E}=0$, we can in principle define also Euler potentials $\beta_{1} \in R$ and $\beta_{2} \in R$ through

$$
\mathbf{E}=\nabla \beta_{2} \times \nabla \beta_{1}
$$

so that the electric lines at time $t_{0}$ are the intersections of the electric surfaces $\beta_{1}\left(\mathbf{r}, t_{0}\right)=k_{1}^{\prime}, \beta_{2}\left(\mathbf{r}, t_{0}\right)=k_{2}^{\prime}$.

It can be seen that the complex scalar fields $\phi$ and $\theta(30)$ define Euler potentials (and canonical variables) of the magnetic and the electric field, respectively, and consequently give the magnetic and the electric lines at every instant of time [34]. Let us begin with the magnetic field equation in (30). Since $\phi$ is a complex field, we define its modulus $|\phi|=\sqrt{(\Re(\phi))^{2}+(\Im(\phi))^{2}}$ and argument $\mathcal{A}_{\phi}=\arctan (\Im(\phi) / \Re(\phi))$, so that

$$
\phi=|\phi| e^{i \mathcal{A}_{\phi}} .
$$

Then, by (30),

$$
\mathbf{B}(\mathbf{r}, t)=\frac{\sqrt{a}}{2 \pi i} \frac{\nabla \phi \times \nabla \bar{\phi}}{(1+\bar{\phi} \phi)^{2}}=\sqrt{a} \nabla\left(\frac{1}{1+|\phi|^{2}}\right) \times \nabla\left(\frac{\mathcal{A}_{\phi}}{2 \pi}\right) .
$$

This means that the real variables

$$
\begin{array}{r}
\alpha_{1}(\mathbf{r}, t)=\frac{1}{1+|\phi|^{2}}, \\
\alpha_{2}(\mathbf{r}, t)=\frac{\mathcal{A}_{\phi}}{2 \pi},
\end{array}
$$

are Euler potentials of the magnetic field. The constant $\sqrt{a}$ in (62) does not play any role in the definition of magnetic lines.

For the electric field, we define the modulus of the complex field $\theta$ as $|\theta|=$ $\sqrt{(\Re(\theta))^{2}+(\Im(\theta))^{2}}$ and its argument as $\mathcal{A}_{\theta}=\arctan (\Im(\theta) / \Re(\theta))$. Putting these into equation (30),

$$
\mathbf{E}(\mathbf{r}, t)=\frac{\sqrt{a} c}{2 \pi i} \frac{\nabla \bar{\theta} \times \nabla \theta}{(1+\bar{\theta} \theta)^{2}}=-c \sqrt{a} \nabla\left(\frac{1}{1+|\theta|^{2}}\right) \times \nabla\left(\frac{\mathcal{A}_{\theta}}{2 \pi}\right) .
$$

The Euler potentials of this electric field are

$$
\begin{array}{r}
\beta_{1}(\mathbf{r}, t)=\frac{1}{1+|\theta|^{2}}, \\
\beta_{2}(\mathbf{r}, t)=\frac{\mathcal{A}_{\theta}}{2 \pi} .
\end{array}
$$


The magnetic lines are then given by the equations $\alpha_{1}=k_{1}$ and $\alpha_{2}=k_{2}$. From (63), these two real equations can be written as the complex equation

$$
\phi(\mathbf{r}, t)=\phi_{0},
$$

so that the complex scalar field $\phi$ gives all the magnetic lines as its level curves. The constant $\phi_{0}$ may be chosen to take any value, in general, in the complex plane. Analogously, the equation

$$
\theta(\mathbf{r}, t)=\theta_{0},
$$

gives all the electric lines when the constant $\theta_{0}$ is set to take values in the complex plane. The complex scalar field $\theta$ is then the complex function whose level curves are the electric lines for every time.

Consequently, if an electromagnetic field can be written as functions of the two complex scalar fields $\phi$ and $\theta$, it allows to study directly the magnetic and the electric lines for every time, and moreover define canonical variables and Euler potentials for the magnetic and the electric fields.

\subsection{Topologically trivial examples}

390

We will see now how some simple examples of electromagnetic fields can be written with the form of equations (30) and (31). It is easier to find the complex scalar fields $\phi$ and $\theta$ when we use the Euler potentials (63) and (65) to write the magnetic and electric fields as

$$
\begin{aligned}
& \mathbf{B}(\mathbf{r}, t)=\sqrt{a} \nabla \alpha_{1} \times \nabla \alpha_{2}=\frac{\sqrt{a}}{c}\left(\frac{\partial \beta_{2}}{\partial t} \nabla \beta_{1}-\frac{\partial \beta_{1}}{\partial t} \nabla \beta_{2}\right), \\
& \mathbf{E}(\mathbf{r}, t)=c \sqrt{a} \nabla \beta_{2} \times \nabla \beta_{1}=\sqrt{a}\left(\frac{\partial \alpha_{2}}{\partial t} \nabla \alpha_{1}-\frac{\partial \alpha_{1}}{\partial t} \nabla \alpha_{2}\right),
\end{aligned}
$$

where $\alpha_{1}$ and $\alpha_{2}$ are given by (63), and $\beta_{1}$ and $\beta_{2}$ are given by (65). From these

Euler potentials, the scalar fields $\phi$ and $\theta$ are given by

$$
\begin{gathered}
\phi=|\phi| e^{i \mathcal{A}_{\phi}}=\sqrt{\frac{1-\alpha_{1}}{\alpha_{1}}} e^{2 \pi i \alpha_{2}}, \\
\theta=|\theta| e^{i \mathcal{A}_{\theta}}=\sqrt{\frac{1-\beta_{1}}{\beta_{1}}} e^{2 \pi i \beta_{2}} .
\end{gathered}
$$


The first example to consider will be the sinusoidal plane wave propagating along the $x$-axis. For a plane wave propagating along the $x$-axis, the electromagnetic field is given by the equations

$$
\begin{gathered}
\mathbf{E}=c B_{0} \sin k(x-c t) \mathbf{u}_{y}, \\
\mathbf{B}=B_{0} \sin k(x-c t) \mathbf{u}_{z} .
\end{gathered}
$$

From (68), the Euler potentials are [35]

$$
\begin{array}{r}
\alpha_{1}=\frac{1}{2}[1-\cos k(x-c t)], \\
\alpha_{2}=\frac{2 B_{0}}{k \sqrt{a}} y, \\
\beta_{1}=\frac{1}{2}[1-\cos k(x-c t)], \\
\beta_{2}=\frac{2 B_{0}}{k \sqrt{a}} z .
\end{array}
$$
fields are

$$
\begin{aligned}
\phi & =\sqrt{\frac{1+\cos k(x-c t)}{1-\cos k(x-c t)}} e^{i\left(4 \pi B_{0} / k \sqrt{a}\right) y}, \\
\theta & =\sqrt{\frac{1+\cos k(x-c t)}{1-\cos k(x-c t)}} e^{i\left(4 \pi B_{0} / k \sqrt{a}\right) z} .
\end{aligned}
$$

The second example to consider is the Coulomb electrostatic field produced by a point charge. The field produced by a point charge $q$ at rest in the origin is given by

$$
\begin{array}{r}
\mathbf{E}=\frac{q}{4 \pi \varepsilon_{0}} \frac{x \mathbf{u}_{x}+y \mathbf{u}_{y}+z \mathbf{u}_{z}}{r^{3}}, \\
\mathbf{B}=0,
\end{array}
$$

where $r=\sqrt{x^{2}+y^{2}+z^{2}}$. Note that this Coulomb field is a solution of Maxwell 410 equations in vacuum in the space $R^{3}$ minus the point in which the charge $q$ is, 
which is diffeomorphic to $S^{2} \times R$. As it was given in [35], the Euler potentials are

$$
\begin{array}{r}
\alpha_{1}=\frac{r}{r+c t}, \\
\alpha_{2}=\frac{q}{4 \pi \varepsilon_{0} c \sqrt{a}}\left(1+\frac{c t}{r}\right) \ln \left(\frac{r}{r_{0}}\right), \\
\beta_{1}=\frac{r+z}{2 r}, \\
\beta_{2}=\frac{q}{2 \pi \varepsilon_{0} c \sqrt{a}} \arctan \left(\frac{y}{x}\right),
\end{array}
$$

where $r_{0}$ is a constant with length dimensions. The static Euler potentials $\beta_{1}$ and $\beta_{2}$ have been used in [38] to illustrate a topological mechanism of quantization of the electric charge. We will not discuss this mechanism but we can point out that $\beta_{2}$ is a phase function. To be well defined in all the space $R^{3}-\{0\}=S^{2} \times R$, the following condition can be imposed,

$$
\frac{q}{\varepsilon_{0} c \sqrt{a}}=N
$$

where $N$ is an integer number so that a quantized value of the electric charge at rest is obtained. As an example, when one takes $N=1$ in the equation (75), the static complex scalar $\theta$ corresponding to a Coulomb field created by a point charge $q=\varepsilon_{0} c \sqrt{a}$ at rest in the origin is

$$
\theta=\sqrt{\frac{r-z}{r+z}}\left(\frac{x}{\sqrt{x^{2}+y^{2}}}+i \frac{y}{\sqrt{x^{2}+y^{2}}}\right)=\frac{x+i y}{r+z} .
$$

The electric surfaces of this example are given by the equations $x=k_{1}(r+z)$ and $y=k_{2}(r+z)$. The surfaces with $k_{1}=k_{2}=1$ have been plotted in Fig. 2 . The intersection of both surfaces is the electric straight line $x=y=-2 z$ and the z-axis.

\subsection{Local equivalence of Rañada construction and decomposable solutions of} Maxwell equations in vacuum

We have seen some examples in which the pair of complex scalar fields $\phi$ and $\theta$ generate an electromagnetic field. We will see that any decomposable 

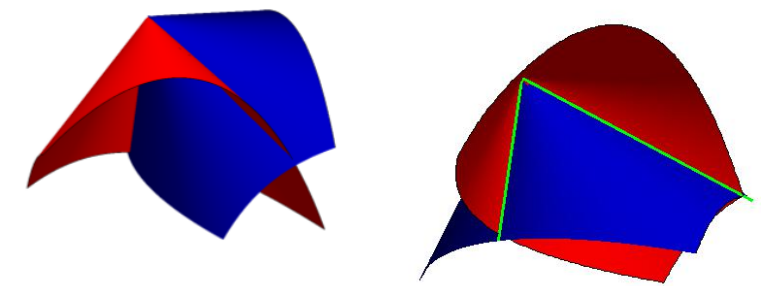

Figure 2: Two views of two electric surfaces given by the static complex scalar field (76). These particular cases are given by the equations $x=r+z$ and $y=r+z$. They intersect along two straight lines, that are also the electric lines of the Coulomb field given by (73). The equation of these electric lines are $x=y=-2 z$, and the $x=y=0$, which is the $\mathrm{z}$-axis.

electromagnetic field can be written locally in terms of this scalar pair, which has the consequence that the set of fields given by (30) and (31) is locally equivalent to the set of solutions of Maxwell equations in vacuum.

To understand the local equivalence, we follow the explanation in [35]. Let us consider the electromagnetic 2-form $F^{d}$ of a decomposable solution of Maxwell equations in vacuum (the superindex $d$ stands for decomposable in this notation, which implies that $\mathbf{E} \cdot \mathbf{B}=0$ ). As we have seen above, this 2 -form can be written locally in terms of canonical variables as in equation (51),

$$
F^{d}=d q \wedge d p
$$

At the same time, from equation (68), the electromagnetic 2-form $F^{R}$ of the EM field constructed from the complex scalar field $\phi$ can be written globally as

$$
F^{R}=\sqrt{a} d \alpha_{2} \wedge d \alpha_{1}
$$

where

$$
\begin{array}{r}
\alpha_{1}=\frac{1}{1+|\phi|^{2}}, \\
\alpha_{2}=\frac{\arctan (\Im(\phi) / \Re(\phi))}{2 \pi} .
\end{array}
$$

The following property is satisfied by the 2 -form $F^{d}$ of a decomposable electromagnetic field in vacuum (the same could be proved for the dual 2 -form ${ }^{*} F^{d}$ ). 
Any decomposable electromagnetic field in vacuum, given by the 2 -form $F^{d}$ and regular in a bounded space-time domain $D$, coincides locally with a field given by (30) and (31) around any point $P \in D$ in the following sense: there is a field 430 given by (30) and (31) with two-form $F^{R}$, such that $F^{d}=F^{R}$ around $P$, except perhaps if $P$ is in a zero measure set. This means that the difference between the set of decomposable solutions of Maxwell equations in vacuum and the set of fields (30) and (31) is not local but global.

To show this property, take the following canonical transformation of the ${ }_{435}$ variables $(q, p)$ in $(77)$,

$$
\begin{gathered}
\eta=\frac{\pi}{\sqrt{a}}\left(p^{2}+q^{2}\right), \\
\delta=\frac{1}{2 \pi} \arctan \left(\frac{q}{p}\right) .
\end{gathered}
$$

Then, we can write

$$
F^{d}=\sqrt{a} d \delta \wedge d \eta
$$

This means that $\eta$ and $\delta$ give another choice of the canonical representation of the decomposable field. Since the 2 -form $F^{R}$ can be written as in (78), we can easily see that $F^{d}=F^{R}$ if there is a complex scalar field $\phi$ so that the following equations hold,

$$
\begin{array}{r}
\delta=\alpha_{2}=\frac{\arctan (\Im(\phi) / \Re(\phi))}{2 \pi}, \\
\eta=\alpha_{1}=\frac{1}{1+|\phi|^{2}} .
\end{array}
$$

The first of these equations poses no problem because $\delta$ was defined as a phase function in (80). Let us concentrate in the second equation in (82).

It is easy to see that $\alpha_{1}$ satisfies $0 \leq \alpha_{1} \leq 1$. However, $\eta$ is defined in (80) and satisfies $0 \leq \eta$. Consequently, if $\eta \leq 1$ for the decomposable field we are considering, the solution for $\alpha_{1}$ will be trivial, and the decomposable field with ${ }_{445} 2$-form $F^{d}$ will be equal to $F^{R}$. The same happens if $\eta$ is bounded, say if $\eta<A$, because we can then take as canonical variables the pair $\eta^{\prime}=\eta / N, \delta^{\prime}=N \delta, N$ being an integer greater than $A$. 
Let us consider the case in which $\eta$ is not bounded in $D$. Let $\Sigma$ be the set of points in which $\eta$ diverges. Since the equation (81) is valid, $\Sigma$ is a zero measure

\section{Electromagnetic knots from maps $S^{3} \rightarrow S^{2}$}

Electromagnetic fields constructed from two complex scalar fields $\phi$ and $\theta$ through equations (30) and (31) allow to introduce topological properties for the field lines configuration. This is in fact what happens when the scalar fields $\phi(\mathbf{r}, t)$ and $\theta(\mathbf{r}, t)$ satisfy certain boundary conditions so that they can be interpreted as maps from the three-sphere $S^{3}$ to the two-sphere $S^{2}$. We will review these conditions and the consequences of using maps $S^{3} \rightarrow S^{2}$ to build electromagnetic knots. Those maps can be classified in homotopy classes characterised by a topological invariant called the Hopf index. The helicity of electromagnetic knots from $S^{3} \rightarrow S^{2}$ maps are proportional to the Hopf index,

470 so the electromagnetic helicity is topologically quantized and it can be identify as the classical limit of the difference between right- and left-handed photons.

In 1977 A. Trautman made use of the Hopf fibration to find solutions of the Maxwell and Yang-Mills equations [10]. Kamchatnov was able to find magnetic fields with topological properties from the Hopf map in the context of magnetohydrodynamics [11]. We will discuss Trautman solutions in this section, and Kamchatnov contribution in the section devoted to plasma knots. 


\subsection{Trautman solutions}

One of the first applications to electromagnetism of maps from the threesphere to the two-sphere is the paper by Trautman [10]. He showed that the static magnetic field corresponding to a magnetic monopole can be found using the natural connection associated to the principal bundle $S^{3} \rightarrow S^{2}$. Furthermore, he proposed a generalization of the previous result to find topologically nontrivial solutions of the Maxwell and Yang-Mills equations.

Consider a magnetic monopole at rest in the Minkowski space-time. The magnetic field defined by the monopole is of Coulomb type and is not well defined at the point in which the monopole is placed as in the second example of Subsection 2.6. If this point, along with its worldline, is removed to get a space in which the magnetic field is finite, then the Minkowski space-time is diffeomorphic to $R^{2} \times S^{2}$. Trautman then considers bundles over $S^{2}$, and specially the Hopf fibration [54], that can be defined as follows. Take the sphere $S^{3}$. The group $U(1)$ acts on this sphere in a way such that the fibers are circles. The quotient space by this action is the sphere $S^{2}$. We will see the Hopf map in more detail in the following sections.

The Hopf bundle admits a connection in terms of Euler angles $\theta, \phi$. The curvature of this connection can be written as

$$
F=\frac{1}{2} \sin \theta d \phi \wedge d \theta
$$

This 2-form is closed, so that $d F=0$, and satisfies the Bianchi identity $d^{*} F=$ 0 , so it is a solution of the Maxwell equations in vacuum. If we take local coordinates $x=\sin \theta \cos \phi, y=\sin \theta \sin \phi, z=\cos \theta$, the electric field associated to the 2 -form (83) is zero, and the magnetic field is of Coulomb type, thus it describes a magnetic monopole of strength $1 / 2$ (Trautman uses natural units in his work).

The nice relation between the Hopf fibration and the magnetic monopole found by Trautman [10] is generalized in the same work. He considers multidimensional spheres $S^{2 n+1}$ and groups of linear transformations that preserve 
their definition in terms of Cartesian coordinates $u_{1}, \ldots, u_{2 n+2}$, i.e. the equation $u_{1}^{2}+\ldots+u_{2 n+2}^{2}=1$. One example is the sphere $S^{5}$ and the group $U(1)$. The quotient space by the action of this group is the 2-dimensional complex projective space $\mathrm{CP}_{2}$. The natural connection defines then, in local coordinates of $C P_{2}$,

$$
\begin{aligned}
& z_{1}=e^{i \mu} \tan \theta \cos \phi, \\
& z_{1}=e^{i \nu} \tan \theta \sin \phi,
\end{aligned}
$$

a closed 2-form $F$ given by

$$
F=\sin 2 \theta d \theta \wedge\left(\cos ^{2} \phi d \mu+\sin ^{2} \phi d \nu\right)-\sin ^{2} \theta \sin 2 \phi d \phi \wedge(d \mu-d \nu),
$$

that satisfies the Bianchi identity and is at the same time self-adjoint, so ${ }^{*} F=F$. This solution of the Maxwell equations in vacuum is called electromagnetic instanton.

\subsection{Some properties of maps from $S^{3} \rightarrow S^{2}$}

Consider a complex field $\phi(\mathbf{r}, t)$. It is a map from the four-dimensional Minkowski space $R^{3} \times R$, where $R^{3}$ is the physical space and $R$ is the time axis, to the complex plane $C$. Suppose that we impose a restriction in the behaviour of the scalar $\phi$ at infinite distance from the origin of the physical space $R^{3}$. Specifically we impose that $\phi=0$ at infinity, independently of the direction that the infinity is approached. This means that

$$
\lim _{|\mathbf{r}| \rightarrow \infty} \phi(\mathbf{r}, t)=0 .
$$

Note that this condition is not fulfilled by the scalar fields (72) or (76). This is because the electromagnetic fields (70) or (73) have infinite electromagnetic energy. Physically, the condition (86) is taken so that the energy of the EM fields (30) and (31) is finite. From a mathematical point of view, the condition (86) identifies all the points at the infinity as a unique point, which is called a compactification at infinity of the space $R^{3}$ by $\phi$. The complex field $\phi$ with this condition can be seen as a map $S^{3} \times R \rightarrow C$. 
We will impose a second condition on $\phi$ which is related to the compactifica-

tion of the complex plane $C$. We can compactify the complex plane at infinity imposing that the inverse images of $\phi=\infty$, which are points in $R^{3}$, do not depend on the direction approaching to infinity in $C$. When this compactification is imposed, the scalar field $\phi$ take values in the sphere $S^{2}$.

Consequently, the complex scalar field $\phi$ with the above restrictions can be seen as a map $S^{3} \times R \rightarrow S^{2}$. Using stereographic projections we can achieve the compactification conditions.

- Compactifying $R^{3}$ to $S^{3}$. The Cartesian coordinates $(x, y, z)$ of a point $P \in R^{3}$ can be related to four real coordinates $\left(u_{1}, u_{2}, u_{3}, u_{4}\right)$ which are Cartesian coordinates in $S^{3}$ if $u_{1}^{2}+u_{2}^{2}+u_{3}^{2}+u_{4}^{2}=1$. The explicit expressions (projecting from the North pole of the sphere) are

$$
u_{1}=\frac{2 x}{r^{2}+1}, u_{2}=\frac{2 y}{r^{2}+1}, u_{3}=\frac{2 z}{r^{2}+1}, u_{4}=\frac{r^{2}-1}{r^{2}+1},
$$

where $r^{2}=x^{2}+y^{2}+z^{2}$.

- The complex plane $C$ is compactified to $S^{2}$. Any point in $C$ can be given by a complex number $\zeta$. This complex number is related to three real coordinates $\left(n_{1}, n_{2}, n_{3}\right)$ which are Cartesian coordinates in $S^{2}$ if $n_{1}^{2}+n_{2}^{2}+$ $n_{3}^{2}=1$. Again the projection from the North pole gives

$$
n_{1}=\frac{2 \Re(\zeta)}{\bar{\zeta} \zeta+1}, n_{2}=\frac{2 \Im(\zeta)}{\bar{\zeta} \zeta+1}, n_{3}=\frac{\bar{\zeta} \zeta-1}{\bar{\zeta} \zeta+1} .
$$

\subsection{The Hopf index and the homotopy classes}

Consider a map $f: S^{3} \rightarrow S^{2}$ written as a complex scalar field $f(\mathbf{r})$ using equations (87) and (88). According to the Hopf theorem [54, 55], if the map $f$ is smooth, the inverse image of any two distinct points $\zeta_{1}$ and $\zeta_{2}$ of $S^{2}, f^{-1}\left(\zeta_{1}\right)$ and $f^{-1}\left(\zeta_{2}\right)$, are two disjoint closed curves in $S^{3}$ (or $R^{3} \cup\{\infty\}$ ). The linking number of the curves $f^{-1}\left(\zeta_{1}\right)$ and $f^{-1}\left(\zeta_{2}\right)$ does not depend on the particular pair of points, since by moving continuously from $\left(\zeta_{1}, \zeta_{2}\right)$ to $\left(\zeta_{1}^{\prime}, \zeta_{2}^{\prime}\right)$ the inverse images can neither untie nor tie any further to one another (since, for this to happen, they should have at a certain moment a common point with two 
different images by $f$ ). This linking number is called the Hopf index of the map $f$,

$$
H(f)=L\left(f^{-1}\left(\zeta_{1}\right), f^{-1}\left(\zeta_{2}\right)\right), \forall \zeta_{1} \neq \zeta_{2} \in S^{2} .
$$

If the map evolves continuously (in time or with any other continuous parameter), the Hopf index must keep a constant value. In other words, the linking number is a constant of the motion in any continuous evolution of the map $f$. Thus the set of maps $S^{3} \rightarrow S^{2}$ (the set of complex scalar fields which are onevalued at infinity) can be classified in homotopy classes, each one labelled by an integer number $H(f)$, called the Hopf index, that is a topological invariant.

An equivalent definition of the Hopf index is the following. Let us take the inverse images of two distinct points $\zeta_{1}$ and $\zeta_{2}$ of $S^{2}$. Since they are two disjoint closed curves, their linking number (the Hopf index) is equal to the number of times that one of them, say $f^{-1}\left(\zeta_{2}\right)$, cuts a surface $S_{1}$ bounded by the curve $f^{-1}\left(\zeta_{1}\right)$. This number is equal to the degree of the map $f$ restricted to $S_{1}$, because every point in $S^{2}$ has $H(f)$ inverse images in $S_{1}$ (see [56]). The degree of that map is equal to the integral over $S_{1}$ of the volume 2 -form of $S^{2}$. This allowed Whitehead [57] to write the Hopf index as an integral in $S^{3}$, as we will see. The volume 2-form in $S^{2}$ can be written in terms of the Cartesian coordinates $\left(n_{1}, n_{2}, n_{3}\right)$ with $n_{1}^{2}+n_{2}^{2}+n_{3}^{2}=1$ defined in (88) as

$$
\sigma=\frac{1}{4 \pi} \frac{d n_{1} \wedge d n_{2}}{n_{3}}
$$

so that it is normalized to unity,

$$
\int_{S^{2}} \sigma=1
$$

The volume 2 -form (90) can also be written in terms of the complex number $\zeta$ related to $n_{i}$ through equations (88), as

$$
\sigma=\frac{1}{2 \pi i} \frac{d \zeta \wedge d \bar{\zeta}}{(1+\bar{\zeta} \zeta)^{2}}
$$

Let us consider the pull-back by the map $f: S^{3} \rightarrow S^{2}$, denoted by $f^{p b}$, that, in a natural way, maps forms in $S^{2}$ into forms in $S^{3}$. Taking into account the 
representation (92), the pull-back of the volume 2 -form is simply

$$
f^{p b} \sigma=\frac{1}{2 \pi i} \frac{d f \wedge d \bar{f}}{(1+\bar{f} f)^{2}} .
$$

The 2-form $f^{p b} \sigma$ is closed, since

$$
d\left(f^{p b} \sigma\right)=f^{p b}(d \sigma)=0
$$

where we have used that $d \sigma=0$ because it is a volume form in $S^{2}$. The cohomological properties [55] of $S^{3}$ imply then than $f^{p b} \sigma$ is also an exact form, what means that there is a 1 -form $g$ in $S^{3}$ such that

$$
f^{p b} \sigma=d g
$$

Whitehead showed [57] that the Hopf index $H(f)$ can be written as the integral

$$
H(f)=\int_{S^{3}} g \wedge f^{p b} \sigma .
$$

When the stereographic projection (87) is used to characterize points in $S^{3}$ in terms of coordinates $(x, y, z)$ of $R^{3}$, the pull-back of the volume form $\sigma$ can be written as

$$
f^{p b} \sigma=\frac{1}{4 \pi i} \frac{\partial_{j} f \partial_{k} \bar{f}-\partial_{j} \bar{f} \partial_{k} f}{(1+\bar{f} f)^{2}} d x^{j} \wedge d x^{k} .
$$

This can be expressed as

$$
f^{p b} \sigma=\frac{1}{2} f_{j k} d x^{j} \wedge d x^{k}
$$

where the antisymmetric tensor $f_{j k}$ is

$$
f_{j k}=\frac{1}{2 \pi i} \frac{\partial_{j} f \partial_{k} \bar{f}-\partial_{j} \bar{f} \partial_{k} f}{(1+\bar{f} f)^{2}} .
$$

Now one can simply define a vector field $\mathbf{b}$ in $R^{3}$ as $\mathbf{b}_{i}(\mathbf{r})=\varepsilon_{i j k} f_{j k} / 2$, so that

$$
\mathbf{b}(\mathbf{r})=\frac{1}{2 \pi i} \frac{\nabla f \times \nabla \bar{f}}{(1+\bar{f} f)^{2}}
$$

The fact that $f^{p b} \sigma$ is an exact form simply means that $\mathbf{b}=\nabla \times \mathbf{a}$, where $\mathbf{a}$ is a vector field in $R^{3}$ related to the 1 -form $g$ in (95) by

$$
g=\mathbf{a}_{i} d x^{i}
$$


It is clear from (100) that the vector field $\mathbf{b}$ is tangent to the level curves of the map $f$. The field $\mathbf{b}$ is known as the Whitehead vector. The Hopf index (96) is given in terms of the Whitehead vector as an integral in $R^{3}$,

$$
H(f)=\int d^{3} r \mathbf{a} \cdot \mathbf{b}
$$

We recognize the right-hand side of equations (100) and (102). Equation (100) is, except the constant $\sqrt{a}$, the definition (30) of the magnetic field $\mathbf{B}$ of an electromagnetic knot, and equation (102) is, except constants, its magnetic helicity. These similarities are the reason for the original Rañada's construction [8]. He was able to construct a way to find electromagnetic fields with nontrivial topological properties by choosing for the magnetic and electric fields of an electromagnetic knot equations similar to the Whitehead vector (100) of a map from $S^{3}$ to $S^{2}$.

Let us summarise all that we have for EM fields constructed from maps $S^{3} \rightarrow S^{2}$. We begin by considering two complex scalar fields $\phi(\mathbf{r}, t)$ and $\theta(\mathbf{r}, t)$.

54 These scalar fields satisfy the following conditions:

1. For each instant of time, the value of $\phi$ and $\theta$ at infinity is zero.

2. For each instant of time, the inverse image of the value $\phi=\infty$ does not depend on the particular direction to go to infinity in the complex plane. Same for the image inverse of the value $\theta=\infty$.

50 Then, both scalars can be interpreted, at each instant of time, as maps $S^{3} \rightarrow S^{2}$. The equivalence between coordinates in $C$ and coordinates in $S^{2}$ is given by the stereographic projection (88), and the equivalence between coordinates in $R^{3}$ and coordinates in $S^{3}$ is given by the stereographic projection (87).

If $\phi: S^{3} \rightarrow S^{2}$ is a smooth function, the inverse image, at each instant of time, of every point $a \in C$ by $\phi$ is a closed curve $\phi^{-1}(a) \in R^{3}$. The linking number of two inverse images $\phi^{-1}(a)$ and $\phi^{-1}(b)$ is the same for all the pairs of points $a, b \in C$. This linking number is a homotopy invariant (and, in particular, a time invariant) called the Hopf index $H(\phi)$ of the map $\phi$. Accordingly, if $\theta$ is a smooth function, the inverse images of every pair of points in the complex so plane by $\theta$ are closed curves whose linking number is the Hopf index $H(\theta)$. 
If we define the electric and magnetic field of an electromagnetic field as in equation (30), then all the magnetic (electric) lines are, at each instant of time, closed curves and they are the level curves of the complex field $\phi(\theta)$. The linking number of every pair of magnetic (electric) lines does not depend on the particular pair we choose, and it is given by the Hopf index $H(\phi)$ of the complex field $\phi$, that is an integer number given by the Whitehead integral

$$
H(\phi)=\frac{1}{a} \int d^{3} r \mathbf{A} \cdot \mathbf{B},
$$

where $\mathbf{B}=\nabla \times \mathbf{A}$. Similarly for the electric lines we have the Whitehead integral

$$
H(\theta)=\frac{1}{a c^{2}} \int d^{3} r \mathbf{C} \cdot \mathbf{E}
$$

where $\mathbf{E}=\nabla \times \mathbf{C}$.

\subsection{Topological quantization of the electromagnetic helicity}

The magnetic helicity $h_{m}$ and the electric helicity $h_{e}$ of en EM field constructed through equations (30) and (31) from two complex scalar fields $\phi(\mathbf{r}, t)$ and $\theta(\mathbf{r}, t)$, which satisfy conditions to be considered as maps $S^{3} \rightarrow S^{2}$ at each instant of time, are given by

$$
\begin{aligned}
h_{m} & =\frac{1}{2 c \mu_{0}} \int d^{3} r \mathbf{A} \cdot \mathbf{B}=\frac{a}{2 c \mu_{0}} H(\phi), \\
h_{e} & =\frac{1}{2 c^{3} \mu_{0}} \int d^{3} r \mathbf{C} \cdot \mathbf{E}=\frac{a}{2 c \mu_{0}} H(\theta),
\end{aligned}
$$

where (103) and (104) have been used. Equations (105) give also the meaning of the constant $a$. Since the Hopf index is dimensionless, the constant $a /\left(c \mu_{0}\right)$ is simply the unit of helicity for the EM field.

To investigate the conservation of magnetic and electric helicities we can take the time derivatives of the helicities. If it is imposed the condition that the fields have finite electromagnetic energy, we get

$$
\begin{aligned}
\frac{d h_{m}}{d t} & =-\frac{1}{c \mu_{0}} \int d^{3} r \mathbf{E} \cdot \mathbf{B} \\
\frac{d h_{e}}{d t} & =\frac{1}{c \mu_{0}} \int d^{3} r \mathbf{E} \cdot \mathbf{B} .
\end{aligned}
$$


Since in the Rañada construction $\mathbf{E} \cdot \mathbf{B}=0$ for every time, the magnetic and electric helicities will be constant.

The electromagnetic helicity $h_{e m}$ is defined only for electromagnetic fields in vacuum and it is the sum of the electric and magnetic ones. From expressions (106), we can see that $h_{e m}=h_{e}+h_{m}$ is a constant independently of the value of $\mathbf{E} \cdot \mathbf{B}$.

Using a decomposition of electromagnetic fields in circularly polarised waves with components $a_{R}(\mathbf{k}), a_{L}(\mathbf{k})$, the electromagnetic helicity $h_{e m}$ can be written as $[37]$

$$
h_{e m}=h_{m}+h_{e}=\hbar \int d^{3} k\left(\bar{a}_{R}(\mathbf{k}) a_{R}(\mathbf{k})-\bar{a}_{L}(\mathbf{k}) a_{L}(\mathbf{k})\right),
$$

where $\hbar$ is the Planck constant. In Quantum Electrodynamics, the integral in the right hand side of equation (107) is interpreted as the helicity operator, that subtracts the number of left-handed photons from the number of right-handed photons. From the usual expressions [58]

$$
\begin{aligned}
& N_{R}=\int d^{3} k \bar{a}_{R}(\mathbf{k}) a_{R}(\mathbf{k}), \\
& N_{L}=\int d^{3} k \bar{a}_{L}(\mathbf{k}) a_{L}(\mathbf{k}),
\end{aligned}
$$

we can write (107) as

$$
h_{e m}=\hbar\left(N_{R}-N_{L}\right) .
$$

Thus the electromagnetic helicity is the classical limit of the difference between the numbers of right-handed and left-handed photons [59,60, 15]. Equation ${ }_{585}$ (109) relates the wave and particle aspects of the electromagnetic helicity. The wave helicity is the sum of the electric and magnetic helicities and characterises the topology of the field lines as a function of the mean linking number of electric lines and magnetic lines. The particle aspect of the electromagnetic helicity is the classical limit of the difference between right- and left-handed photons. An electromagnetic field with a trivial configuration of force lines (zero linking number), is such that the classical expression for the number of right-handed photons is equal to the classical expression for the number of lefthanded photons. On the opposite, a non-zero linking number of field lines 
indicates that the numbers of right-handed photons and left-handed photons will be classically different.

The electromagnetic helicity for an electromagnetic knot constructed from maps $S^{3} \rightarrow S^{2}$ is given by

$$
h_{e m}=h_{m}+h_{e}=\frac{a}{2 c \mu_{0}}[H(\phi)+H(\theta)] .
$$

This means that, for electromagnetic knots from maps $S^{3} \rightarrow S^{2}$, we arrive at the relation

$$
N_{R}-N_{L}=\frac{a}{2 \hbar c \mu_{0}}[H(\phi)+H(\theta)] .
$$

This equation has been called the topological quantization of the electromagnetic helicity [37] since it relates the helicity to a pair of integer numbers that are Hopf indices of maps $S^{3} \rightarrow S^{2}$ used to build the magnetic and electric fields.

It is clear that not all the Rañada fields come from maps $S^{3} \rightarrow S^{2}$. We have previously seen examples in which $\phi$ and $\theta$ do not satisfy conditions to be considered as such kind of maps between spheres (see equations (72) and (76)). However, the Rañada fields that come from maps $S^{3} \rightarrow S^{2}$ show very interesting topological properties that have a reflection in their physical behaviour. In particular, their (magnetic or electric) field lines form links and knots, and their link and knot type appears to be the same for all the curves and for every time. This topological constraint can be used in interesting applications, as we will see later. And this is the main reason for the search of explicit examples of these fields.

\section{The electromagnetic Hopfion}

The first topologically nontrivial solution was based in the Hopf fibration and can be called the Hopf-Rañada electromagnetic knot [8, 9, 32, 33, 15, 35, 17, 28]. In a more general context, this solution is also known as the electromagnetic Hopfion. In 2009, Besieris and Shaarawi showed [61] that the Hopfion is equivalent to the Robinson congruence [62], used in gauge theories by Trautman [10] and well studied by Bialynicki-Birula [63]. 
As mentioned previously, the term knot is a bit confusing for the Hopfion, since the field lines are isomorphic to linked circles, which can be considered rather trivial knots. However, those solutions are given by two complex scalar fields as defined by Rañada as we will see.

The explicit solution of the time-dependent scalar fields $\phi(\mathbf{r}, t), \theta(\mathbf{r}, t)$ that satisfy equations (30) and (31) for the electromagnetic Hopfion was obtained in [15] and can be written as

$$
\begin{aligned}
\phi & =\frac{(A X-T Z)+i(A Y+T(A-1))}{(A Z+T X)+i(A(A-1)-T Y)}, \\
\theta & =\frac{(A Y+T(A-1))+i(A Z+T X)}{(A X-T Z)+i(A(A-1)-T Y)} .
\end{aligned}
$$

In these expressions, coordinates $(X, Y, Z, T)$ are dimensionless to be sure that the complex scalar fields $\phi$ and $\theta$ are also dimensionless. In this way, they can be used in applications covering different length scales. From the Minkowski space coordinates $(x, y, z, t)$, upper-case coordinates are defined as

$$
X=\frac{x}{L_{0}}, Y=\frac{y}{L_{0}}, Z=\frac{z}{L_{0}}, T=\frac{c t}{L_{0}},
$$

where $L_{0}$ is a characteristic length size of the scalar fields. Equations (113) are supplemented with

$$
R=\frac{r}{L_{0}}=\frac{\sqrt{x^{2}+y^{2}+z^{2}}}{L_{0}}=\sqrt{X^{2}+Y^{2}+Z^{2}} .
$$

In the expressions (112), the quantity $A$ (not to be confused with the modulus of a vector potential $\mathbf{A}$ ) is

$$
A=\frac{R^{2}-T^{2}+1}{2}
$$

The magnetic and electric fields corresponding to the scalar fields (112) are computed using (30) and (31). The result is

$$
\begin{aligned}
\mathbf{B}(\mathbf{r}, t) & =\frac{\sqrt{a}}{\pi L_{0}^{2}} \frac{Q \mathbf{H}_{1}+P \mathbf{H}_{2}}{\left(A^{2}+T^{2}\right)^{3}}, \\
\mathbf{E}(\mathbf{r}, t) & =\frac{c \sqrt{a}}{\pi L_{0}^{2}} \frac{Q \mathbf{H}_{2}-P \mathbf{H}_{1}}{\left(A^{2}+T^{2}\right)^{3}} .
\end{aligned}
$$


where the quantities $P, Q$ are defined by

$$
P=T\left(T^{2}-3 A^{2}\right), Q=A\left(A^{2}-3 T^{2}\right),
$$

and the vectors $\mathbf{H}_{1}$ and $\mathbf{H}_{2}$ are,

$$
\begin{aligned}
& \mathbf{H}_{1}=(Y+T-X Z) \mathbf{u}_{x}-(X+(Y+T) Z) \mathbf{u}_{y}+\left(\frac{-1-Z^{2}+X^{2}+(Y+T)^{2}}{2}\right) \mathbf{u}_{z}, \\
& \mathbf{H}_{2}=\left(\frac{1+X^{2}-Z^{2}-(Y+T)^{2}}{2}\right) \mathbf{u}_{x}+(-Z+X(Y+T)) \mathbf{u}_{y}+(Y+T+X Z) \mathbf{u}_{z}(1
\end{aligned}
$$

\subsection{Euler potentials of the Hopfion}

In order to study the magnetic and electric surfaces created by this electromagnetic field, and its magnetic and electric field lines, we use Euler potentials as in the equations (68), so that

$$
\begin{aligned}
& \mathbf{B}(\mathbf{r}, t)=\sqrt{a} \nabla \alpha_{1} \times \nabla \alpha_{2}, \\
& \mathbf{E}(\mathbf{r}, t)=c \sqrt{a} \nabla \beta_{2} \times \nabla \beta_{1} .
\end{aligned}
$$

The Euler potentials for the electromagnetic knot (112) are

$$
\begin{aligned}
& \alpha_{1}(\mathbf{r}, t)=\frac{(A Z+T X)^{2}+(A(A-1)-T Y)^{2}}{\left(A^{2}+T^{2}\right)^{2}}, \\
& \alpha_{2}(\mathbf{r}, t)=\frac{1}{2 \pi} \arctan \left(\frac{\left(A^{2}-T^{2}\right)(Y Z-(A-1) X)+2 A T(X Y+(A-1) Z)}{\left(A^{2}-T^{2}\right)(X Z+(A-1) Y)+A T\left((A-1)^{2}+X^{2}-Y^{2}-Z^{2}\right)}\right), \\
& \beta_{1}(\mathbf{r}, t)=\frac{(A X-T Z)^{2}+(A(A-1)-T Y)^{2}}{\left(A^{2}+T^{2}\right)^{2}}, \\
& \beta_{2}(\mathbf{r}, t)=\frac{1}{2 \pi} \arctan \left(\frac{\left(A^{2}-T^{2}\right)(X Z-(A-1) Y)+A T\left(X^{2}+Y^{2}-Z^{2}-(A-1)^{2}\right)}{\left(A^{2}-T^{2}\right)(X Y+(A-1) Z)+2 A T((A-1) X-Y Z)}\right) .
\end{aligned}
$$

The first two of these Euler potentials give the magnetic surfaces and the intersections of each pair of magnetic surfaces are the magnetic field lines. The last two Euler potentials give the electric surfaces and their intersections are the electric field lines. Using the alternative formalism of geometric algebra, Van Enk has shown this particular choice of the Euler potentials to be covariant [50].

Consider first the magnetic surfaces. In Fig. 3 we plot some magnetic surfaces given by $\alpha_{1}(\mathbf{r}, t)=k_{1}$ at instant of time $t=0$, where $0 \leq k_{1} \leq 1$. These surfaces satisfy the equation

$$
\left(1-k_{1}\right) R^{4}-2\left(1+k_{1}\right) R^{2}+4 Z^{2}+\left(1-k_{1}\right)=0 .
$$


Figure 3: Structure of magnetic surfaces of the electromagnetic Hopfion at $T=0$, given as solutions of the equation $\alpha_{1}(\mathbf{r}, t=0)=k_{1}$, with $0 \leq k_{1} \leq 1$. The surface $\alpha_{1}(\mathbf{r}, t=0)=0$ is singular, and it is the circle $X^{2}+Y^{2}=1$. For $0<k_{1}<1$ we have tori around the circle $x^{2}+y^{2}=1$, each of them covering the previous ones. The points in these tori cover all the space $R^{3}$. In the figure we have plotted the tori $k_{1}=0.001,0.09,0.5,0.9995$. When $k_{1}$ approaches the value $k_{1}=1$ the radii of the torus approach infinity (see the surface $k_{1}=0.9995$ ), and we have another singular surface, that is the $Z$-axis.

The magnetic surfaces for $k_{1}=0$ and $k_{1}=1$ are extreme cases, and they are singular. For $k_{1}=1$, we have the $Z$-axis, and for $k_{1}=0$ we have the curve $X^{2}+Y^{2}=1$. Both are magnetic lines. For $0<k_{1}<1$, the rest of magnetic ${ }_{640}$ surfaces $\alpha_{1}(\mathbf{r}, t=0)=k_{1}$ are nested tori between the singular extreme cases $k_{1}=0$ and $k_{1}=1$. This structure means that the magnetic lines of the magnetic field (116) at $t=0$ lie on tori.

The magnetic surfaces $\alpha_{2}(\mathbf{r}, t)=k_{2}$ at $T=0$ are given by

$$
\frac{2 Y Z-X\left(R^{2}-1\right)}{2 X Z+Y\left(R^{2}-1\right)}=\tan \left(2 \pi k_{2}\right)
$$

for different values of $k_{2}$. The surfaces given by equation (122) are more complicated than the tori of equation (121). Some examples are plotted in Fig. 4.

${ }_{645}$ The magnetic lines at $T=0$ are the intersections of the surfaces $\alpha_{1}(\mathbf{r}, 0)=k_{1}$ with the surfaces $\alpha_{2}(\mathbf{r}, 0)=k_{2}$. Each one of these intersections is a closed curve in $R^{3}$, and this curve is topologically equivalent to a circle (see left pannel in Fig. 6). The set of all these magnetic lines is known as the Hopf fibration [54]. 
Figure 4: The magnetic surface of the electromagnetic Hopfion at $T=0$, given as solution of the equation $\alpha_{2}(\mathbf{r}, t=0)=k_{2}$ with $k_{2}=-1 / 2,0,1 / 2$.

The structure of the magnetic surfaces is topologically conserved as time evolves. In Fig. 5 we have plotted some magnetic surfaces $\alpha_{1}(\mathbf{r}, T=0.4)=k_{1}$ as an example. The structure of the magnetic lines is also conserved as can be seen in the right pannel of Fig. 6.

At $t=0$, the electric surfaces given by $\beta_{1}(\mathbf{r}, t=0)=k_{1}^{\prime}$, with $0 \leq k_{1}^{\prime} \leq 1$, satisfy

$$
\left(1-k_{1}^{\prime}\right) R^{4}-2\left(1+k_{1}^{\prime}\right) R^{2}+4 X^{2}+\left(1-k_{1}^{\prime}\right)=0 .
$$

Note that this equation is very similar to the equation (121) for the magnetic lines at $T=0$, with the change $Z \rightarrow X$. This means that the electric surfaces $\beta_{1}(\mathbf{r}, t=0)=k_{1}^{\prime}$ can be given as a $\pi / 2$-rotation of the magnetic surfaces $\alpha_{1}(\mathbf{r}, t=0)=k_{1}^{\prime}$ around the $Y$-axis. Consequently, magnetic surfaces and electric surfaces are orthogonal at each point of $R^{3}$. For $k_{1}^{\prime}=1$, we have a singular electric surface that is the $X$-axis. For $k_{1}^{\prime}=0$ we have another singular surface, that is the circle $Y^{2}+Z^{2}=1$. For $0<k_{1}^{\prime}<1$, the rest of electric surfaces $\beta_{1}(\mathbf{r}, t=0)=k_{1}^{\prime}$ are nested tori. The overall structure is the same as in Fig. 3 with a $\pi / 2$ rotation around the $Y$-axis. At $t=0$, the electric surfaces $\beta_{2}(\mathbf{r}, t=0)=k_{2}^{\prime}$ are given by

$$
\frac{2 X Z-Y\left(R^{2}-1\right)}{2 X Y+Z\left(R^{2}-1\right)}=\tan \left(2 \pi k_{2}^{\prime}\right)
$$


Figure 5: Structure of magnetic surfaces of the electromagnetic Hopfion at $T=0.4$ as solutions of the equation $\alpha_{1}=k_{1}$. The overall topological structure is the same as the one in Fig. 4 but the surfaces have been smoothly deformed. We plot the cases $k_{1}=0.001,0.09,0.5$.

Again, this equation is similar to (123) with the changes $X \rightarrow Y, Y \rightarrow Z$, $Z \rightarrow X$. This transformation is a positive permutation of coordinates $(X, Y, Z)$. An example of these electric surfaces is then the one given in Fig. 4 with a positive permutation of the $R^{3}$-coordinates. The intersection of a pair of electric surfaces, one of them given by (123) and the other given by (124), is topologically equivalent to a circle, the set of all the electric lines is also a Hopf fibration, whose structure is conserved as time evolves, and the electric line at a given point is then orthogonal to the magnetic line at the same point.

However, note that not all the electric surfaces can be obtained from the magnetic surfaces by a positive permutation of coordinates $(X, Y, Z)$ when $T \neq 0$. This only happens with $T=0$. To verify it, one simply makes the permutation in $\alpha_{1}$ and $\alpha_{2}$ and compare the result with $\beta_{1}$ and $\beta_{2}$ in equation (120). Nev665 ertheless at every time the magnetic and electric surfaces are similar and they are orthogonal to each other at every point of $R^{3}$, so the magnetic lines of the electromagnetic Hopfion are always orthogonal to the electric lines. 

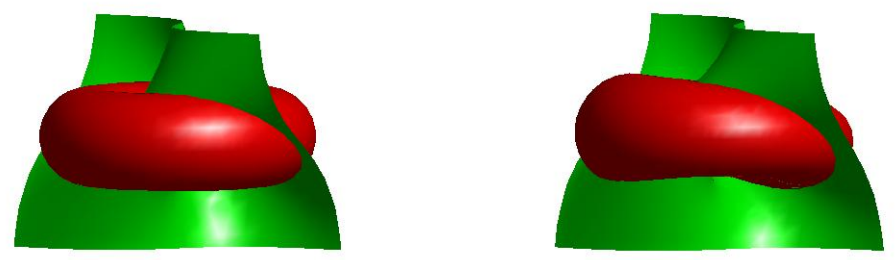

Figure 6: Structure of magnetic lines of the electromagnetic Hopfion. The intersections of magnetic isosurfaces are magnetic lines. Left at $T=0, \alpha_{1}=0.3, \alpha_{2}=0$. Right the same at $T=0.2$.

\subsection{The fibration of the Hopfion}

The structure of all the magnetic lines at a given instant of time, and also the structure of all the electric lines at a given instant of time, can be understood by considering the scalar fields (112) as maps $S^{3} \times R \rightarrow S^{2}$. To do that, we write the scalar fields as

$$
\begin{aligned}
\phi & =\frac{u_{1}+i u_{2}}{u_{3}+i u_{4}}, \\
\theta & =\frac{u_{2}+i u_{3}}{u_{1}+i u_{4}},
\end{aligned}
$$

where the quantities

$$
\begin{aligned}
& u_{1}(\mathbf{r}, t)=\frac{A X-T Z}{\left(A^{2}+T^{2}\right)}, \\
& u_{2}(\mathbf{r}, t)=\frac{A Y+T(A-1)}{\left(A^{2}+T^{2}\right)}, \\
& u_{3}(\mathbf{r}, t)=\frac{A Z+T X}{\left(A^{2}+T^{2}\right)}, \\
& u_{4}(\mathbf{r}, t)=\frac{A(A-1)-T Y}{\left(A^{2}+T^{2}\right)},
\end{aligned}
$$

satisfy $u_{1}^{2}+u_{2}^{2}+u_{3}^{2}+u_{4}^{2}=1$, and can be interpreted as time-dependent coordinates of the sphere $S^{3}$, equation (126) being a time-dependent smooth deformation of the stereographic projection (87). In fact, the exact form of (87) can be obtained by taking $t=0$ in (126). We can also write coordinates in $S^{2}$ from 
the complex field $\phi$ by using the stereographic projection (88). We get

$$
\phi=\frac{n_{1}+i n_{2}}{1-n_{3}},
$$

where $n_{1}^{2}+n_{2}^{2}+n_{3}^{2}=1$, so that $\left(n_{1}, n_{2}, n_{3}\right)$ are coordinates of the sphere $S^{2}$.

These coordinates are given by the inverse relation

$$
\begin{aligned}
& n_{1}=\frac{\phi+\bar{\phi}}{\phi \bar{\phi}+1}, \\
& n_{2}=\frac{-i(\phi-\bar{\phi})}{\phi \bar{\phi}+1}, \\
& n_{3}=\frac{\phi \bar{\phi}-1}{\phi \bar{\phi}+1} .
\end{aligned}
$$

Using (125) and (126), the scalar field $\phi$ can be interpreted as a time-dependent map $S^{3} \rightarrow S^{2}$ that can be expressed as

$$
\begin{aligned}
n_{1} & =2\left(u_{1} u_{3}+u_{2} u_{4}\right) \\
& =\frac{2}{\left(A^{2}+T^{2}\right)^{2}}\left[\left(A^{2}-T^{2}\right)(X Z+Y(A-1))+A T\left(X^{2}-Y^{2}-Z^{2}+(A-1)^{2}\right)\right], \\
n_{2} & =2\left(u_{2} u_{3}-u_{1} u_{4}\right) \\
& =\frac{2}{\left(A^{2}+T^{2}\right)^{2}}\left[\left(A^{2}-T^{2}\right)(Y Z-X(A-1))+2 A T(X Y+Z(A-1))\right], \\
n_{3} & =u_{1}^{2}+u_{2}^{2}-u_{3}^{2}-u_{4}^{2} \\
& =\frac{1}{\left(A^{2}+T^{2}\right)^{2}}\left[\left(A^{2}-T^{2}\right)\left(X^{2}+Y^{2}-Z^{2}-(A-1)^{2}\right)+4 A T(Y(A-1)-X Z)\right] .
\end{aligned}
$$

This is the Hopf map. When $t=0$ we get the more familiar expressions

$$
\begin{aligned}
& n_{1}=\frac{4}{\left(R^{2}+1\right)^{2}}\left[2 X Z+Y\left(R^{2}-1\right)\right], \\
& n_{2}=\frac{4}{\left(R^{2}+1\right)^{2}}\left[2 Y Z-X\left(R^{2}-1\right)\right], \\
& n_{3}=\frac{1}{\left(R^{2}+1\right)^{2}}\left[4 X^{2}+4 Y^{2}-4 Z^{2}-\left(R^{2}-1\right)^{2}\right] .
\end{aligned}
$$

The Hopf structure of the level curves of the scalar field $\phi$ determines the properties of the magnetic lines of the field given(116). The magnetic lines are level curves of $\phi$ and all of them are closed curves topologically equivalent to circles. Moreover, any pair of these magnetic lines is a link, and the linking number of any pair of magnetic lines is equal to 1. In Fig. 1, we have plotted some magnetic lines at $T=0$ and at $T=0.2$. 

a time-dependent map $S^{3} \rightarrow S^{2}$ where

$$
\begin{aligned}
n_{1} & =2\left(u_{1} u_{2}+u_{3} u_{4}\right) \\
& =\frac{2}{\left(A^{2}+T^{2}\right)^{2}}\left[\left(A^{2}-T^{2}\right)(X Y+Z(A-1))+2 A T(X(A-1)-Y Z)\right] \\
n_{2} & =2\left(u_{1} u_{3}-u_{2} u_{4}\right) \\
& =\frac{2}{\left(A^{2}+T^{2}\right)^{2}}\left[\left(A^{2}-T^{2}\right)(X Z-Y(A-1))+A T\left(X^{2}+Y^{2}-Z^{2}-(A-1)^{2}\right)\right], \\
n_{3} & =u_{2}^{2}+u_{3}^{2}-u_{1}^{2}-u_{4}^{2} \\
& =\frac{1}{\left(A^{2}+T^{2}\right)^{2}}\left[\left(A^{2}-T^{2}\right)\left(Y^{2}+Z^{2}-X^{2}-(A-1)^{2}\right)+4 A T(Y(A-1)+X Z)\right] .
\end{aligned}
$$

Again this is the Hopf map. It can be obtained from the map $\phi$ (130) by the changes $u_{1} \rightarrow u_{2}, u_{2} \rightarrow u_{3}, u_{3} \rightarrow u_{1}$. When $t=0$, the map $\theta$ is given by

$$
\begin{aligned}
& n_{1}=\frac{4}{\left(R^{2}+1\right)^{2}}\left[2 X Y+Z\left(R^{2}-1\right)\right], \\
& n_{2}=\frac{4}{\left(R^{2}+1\right)^{2}}\left[2 X Z-Y\left(R^{2}-1\right)\right], \\
& n_{3}=\frac{1}{\left(R^{2}+1\right)^{2}}\left[4 Y^{2}+4 Z^{2}-4 X^{2}-\left(R^{2}-1\right)^{2}\right] .
\end{aligned}
$$

By analogy with the magnetic case, the electric lines are level curves of $\theta$ and all of them are closed curves topologically equivalent to circles. Any pair of electric lines is a link, and the linking number is equal to 1.

The fact that the linking number of any pair of magnetic lines is equal to 1 and the linking number of any pair of electric lines is equal to 1 for the electromagnetic Hopfion has an easy explanation: both the magnetic lines and the electric lines are the level curves of Hopf maps, and the Hopf index of the Hopf map is equal to 1 . Remember that the Hopf index of a map $S^{3} \rightarrow S^{2}$ is the linking number of any pair of its level curves. Consequently, for the Hopfion we have that the Hopf indices of the maps $\phi$ and $\theta$ satisfy

$$
H(\phi)=H(\theta)=1
$$

According to (105), this means that the magnetic and electric helicities of the 
electromagnetic Hopfion are given by

$$
\begin{aligned}
h_{m} & =\frac{1}{2 c \mu_{0}} \int d^{3} r \mathbf{A} \cdot \mathbf{B}=\frac{a}{2 c \mu_{0}} H(\phi)=\frac{a}{2 c \mu_{0}}, \\
h_{e} & =\frac{1}{2 c^{3} \mu_{0}} \int d^{3} r \mathbf{C} \cdot \mathbf{E}=\frac{a}{2 c \mu_{0}} H(\theta)=\frac{a}{2 c \mu_{0}},
\end{aligned}
$$

and the electromagnetic helicity is

$$
h_{e m}=h_{m}+h_{e}=\frac{a}{2 c \mu_{0}}[H(\phi)+H(\theta)]=\frac{a}{c \mu_{0}},
$$

so that the classical limit of the difference between right- and left-handed photons as discussed previously is

$$
N_{R}-N_{L}=\frac{h_{e m}}{\hbar}=\frac{a}{\hbar c \mu_{0}} .
$$

\subsection{Further properties of the Hopfion}

Next we look for some properties of the electromagnetic Hopfion. First, it is easy to see that this solution verifies the null field conditions $\mathbf{E} \cdot \mathbf{B}=0$ and $E^{2}-c^{2} B^{2}=0$. To show these null field conditions, we use equations (116). Then

$$
\begin{aligned}
\mathbf{E} \cdot \mathbf{B} & =\frac{a c}{\pi^{2} L_{0}^{4}\left(A^{2}+T^{2}\right)^{6}}\left[\left(Q^{2}-P^{2}\right) \mathbf{H}_{1} \cdot \mathbf{H}_{2}+P Q\left(H_{2}^{2}-H_{1}^{2}\right)\right]=0,(137) \\
E^{2}-c^{2} B^{2} & =\frac{a c^{2}}{\pi^{2} L_{0}^{4}\left(A^{2}+T^{2}\right)^{6}}\left[\left(Q^{2}-P^{2}\right)\left(H_{2}^{2}-H_{1}^{2}\right)-2 P Q \mathbf{H}_{1} \cdot \mathbf{H}_{2}\right]=0,
\end{aligned}
$$

since, by equation (118), $H_{2}^{2}-H_{1}^{2}=0$ and $\mathbf{H}_{1} \cdot \mathbf{H}_{2}=0$.

The null field conditions satisfied by the electromagnetic Hopfion allows to compute easily the magnetic and electric helicities from the magnetic and electric fields and show that equations (134) hold. First we use equations (106) to show that, for the electromagnetic Hopfion,

$$
\begin{aligned}
\frac{d h_{m}}{d t}=-\frac{1}{c \mu_{0}} \int d^{3} r \mathbf{E} \cdot \mathbf{B} & =0, \\
\frac{d h_{e}}{d t}=\frac{1}{c \mu_{0}} \int d^{3} r \mathbf{E} \cdot \mathbf{B} & =0 .
\end{aligned}
$$


This means that both the magnetic and the electric helicities are constant.

$$
\begin{gathered}
h_{m}=h_{m}(t=0)=\frac{1}{2 c \mu_{0}} \int d^{3} r \mathbf{A}(\mathbf{r}, t=0) \cdot \mathbf{B}(\mathbf{r}, t=0), \\
h_{e}=h_{e}(t=0)=\frac{1}{2 c^{3} \mu_{0}} \int d^{3} r \mathbf{C}(\mathbf{r}, t=0) \cdot \mathbf{E}(\mathbf{r}, t=0) .
\end{gathered}
$$

The magnetic and electric fields at $t=0$ can be taken from (116). We get

$$
\begin{aligned}
& \mathbf{B}(\mathbf{r}, t=0)=\frac{8 \sqrt{a}}{\pi L_{0}^{2}\left(1+R^{2}\right)^{3}}\left[(Y-X Z) \mathbf{u}_{x}-(X+Y Z) \mathbf{u}_{y}+\left(\frac{-1-Z^{2}+X^{2}+Y^{2}}{2}\right) \mathbf{u}_{z}\right] \\
& \mathbf{E}(\mathbf{r}, t=0)=\frac{8 \sqrt{a} c}{\pi L_{0}^{2}\left(1+R^{2}\right)^{3}}\left[\left(\frac{1+X^{2}-Z^{2}-Y^{2}}{2}\right) \mathbf{u}_{x}+(-Z+X Y) \mathbf{u}_{y}+(Y+X Z) \mathbf{u}_{z}\right]
\end{aligned}
$$

From them, the vector potentials such that $\mathbf{B}(\mathbf{r}, t=0)=\nabla \times \mathbf{A}(\mathbf{r}, t=0)$ and $\mathbf{E}(\mathbf{r}, t=0)=\nabla \times \mathbf{C}(\mathbf{r}, t=0)$ can be taken as $[33]$

$$
\begin{aligned}
& \mathbf{A}(\mathbf{r}, t=0)=\frac{2 \sqrt{a}}{\pi L_{0}\left(1+R^{2}\right)^{2}}\left[Y \mathbf{u}_{x}-X \mathbf{u}_{y}-\mathbf{u}_{z}\right] \\
& \mathbf{C}(\mathbf{r}, t=0)=\frac{2 \sqrt{a} c}{\pi L_{0}\left(1+R^{2}\right)^{2}}\left[\mathbf{u}_{x}-Z \mathbf{u}_{y}+Y \mathbf{u}_{z}\right]
\end{aligned}
$$

Writing (140) and (141) into (139), we get

$$
\begin{aligned}
& h_{m}=\frac{1}{2 c \mu_{0}} \int d^{3} r \mathbf{A} \cdot \mathbf{B}=\frac{a}{2 c \mu_{0}}, \\
& h_{e}=\frac{1}{2 c^{3} \mu_{0}} \int d^{3} r \mathbf{C} \cdot \mathbf{E}=\frac{a}{2 c \mu_{0}},
\end{aligned}
$$

for the electromagnetic Hopfion, in agreement with (134), that was obtained using only topological arguments.

The energy density of the electromagnetic Hopfion is given by

$$
U(\mathbf{r}, t)=\frac{\varepsilon_{0} E^{2}}{2}+\frac{B^{2}}{2 \mu_{0}}=\frac{a}{4 \pi^{2} \mu_{0} L_{0}^{4}} \frac{\left(1+X^{2}+(Y+T)^{2}+Z^{2}\right)^{2}}{\left(A^{2}+T^{2}\right)^{3}} .
$$

The maximum of the energy density is located at $X=Z=0$ during the time evolution [28], and $U$ is symmetric in the coordinates $X$ and $Z$. In Fig. 7, we show some level surfaces of the energy density $U$ for different times. $T=0,1.5,2.5$. The energy density levels represented are 0.5, 1 and 1.5 in $a /\left(4 \pi^{2} \mu_{0} L_{0}^{4}\right)$ units. It can be seen how the level surfaces spread as the energy density goes to zero. The total electromagnetic energy of the knot, that is

$$
\mathcal{E}=\int U d^{3} r=\frac{2 a}{\mu_{0} L_{0}}
$$



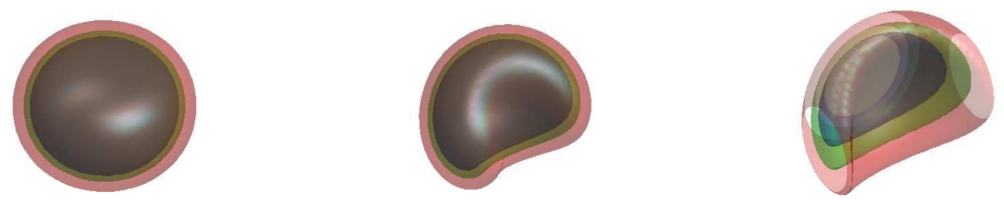

Figure 7: (Colour online) Evolution of energy density levels of the electromagnetic Hopfion. From left to right $T=0, T=1.5, T=2.5$. From outside to inside, the energy levels correspond to values $0.5,1$ and 1.5 (red, green, blue) of the energy density in $a /\left(4 \pi^{2} \mu_{0} L_{0}^{4}\right)$ units.

remains constant. At $T=0$, the energy density has spherical symmetry and its maximum is located at the origin. As time increases, the symmetry is broken and the maximum is numerically located at $X=Z=0$ and $Y$ close to $T$. Approximately, the position of the maximum of the energy density can be found up to $T=1$ at $X=Z=0, Y=T\left(1+6 T^{2}\right) /\left(2+6 T^{2}\right)$.

Following [28], the energy density of the electromagnetic Hopfion extends to infinity, but we can define a mean quadratic radius of the energy distribution as

$$
<r^{2}>=\frac{\int\left(\mathbf{r}-\mathbf{r}_{\max }\right)^{2} U d^{3} r}{\int U d^{3} r},
$$

where $\mathbf{r}_{\max }$ is the position of the maximum of the distribution. At $T=0$, the distribution has spherical symmetry and the mean quadratic radius of the distribution is given by $\sqrt{\left\langle r^{2}\right\rangle}=L_{0}$. The maximum has a value of the energy density equal to $4^{3}$ in $a /\left(4 \pi^{2} \mu_{0} L_{0}^{4}\right)$ units, and at a distance equal to the mean quadratic value, the energy density is 4 in $a /\left(4 \pi^{2} \mu_{0} L_{0}^{4}\right)$ units. This means that, initially, more than $70 \%$ of the energy is localized inside a sphere of radius $L_{0}$ centered at the origin. As time evolves the mean quadratic radius of the distribution spreads out (its value at $t=L_{0} / c$ or $T=1$ is about $1.1 L_{0}$ ) and the position of the center is at $(0,7 / 8,0)$. The distribution is not well characterized as a sphere when $T \neq 0$, taking an umbrella-like shape [17]. 

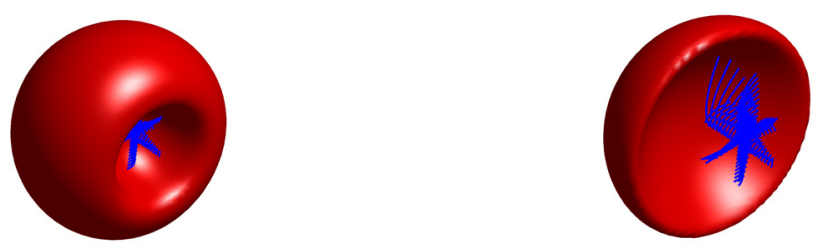

Figure 8: Evolution of the position of electrons under the influence of the electromagnetic Hopfion. From left to right, $T=2, T=4.5$. The energy level shown corresponds to value 0.1 of the density energy in $a /\left(\mu_{0} L_{0}^{4}\right)$ units for the same times. The initial velocity of the electrons is 0 .

The linear momentum of the Hopfion takes the value

$$
\mathbf{p}=\int \varepsilon_{0} \mathbf{E} \times \mathbf{B} d^{3} r=\frac{a}{c \mu_{0} L_{0}} \mathbf{u}_{y}
$$

As can be seen, it has a single contribution along the $y$-axis. This explains why the maximum of the energy density moves along this axis. In Fig. 8 we have plot the motion of test charges under the Hopfion influence. For more details about the motion of the test charges we point the interested reader to [28].

\section{Non-null torus knotted electromagnetic fields}

A new range of solutions covering the topology of the whole torus knots set and having the Hopfion as a particular case, was found [18]. In those solutions initially all the magnetic lines and all the electric lines are linked torus knots. However, for some members of the class, the properties of the Rañada construction are modified so the solutions are non-null fields in general. Although we call them non-null fields, we want to point that in this class there are null fields, the Hopfion for instance, so the class is broader.

Torus knots are knotted curves lying on the surface of a torus. Any torus knot is defined by two coprime integer numbers $(n, m)$ in such a way that the 
$(3,2)$

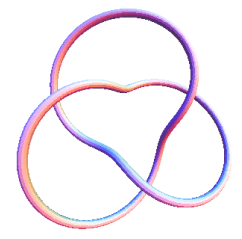

$(5,3)$

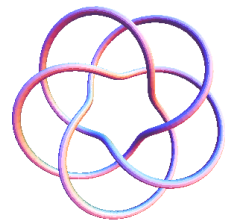

$(7,4)$

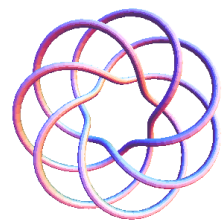

$(4,3)$

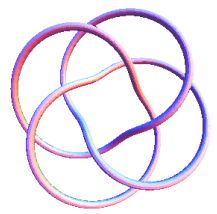

$(5,4)$

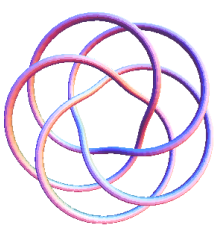

$(8,5)$

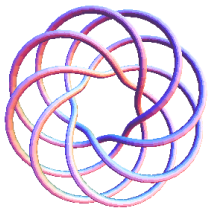

$(5,2)$

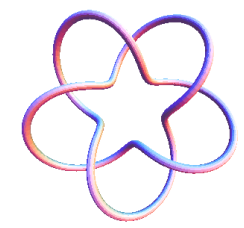

$(7,2)$

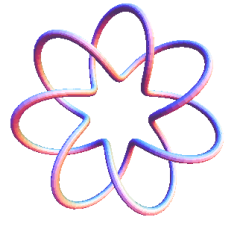

$(8,7)$

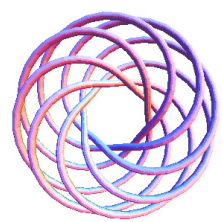

Figure 9: Some $(n, m)$ torus knots. These knotted curves are lying on the surface of a torus in the three-dimensional space $R^{3}$. In the figures, all the torus knots are called nontrivial. If $n$ or $m$ were equal to 1 , the knot is said to be trivial since its representation is a circle, that can be linked to other ones.

curve winds $n$ times around a circle inside the torus and $m$ times around a line through the hole in the torus. In Fig. 9 there are plotted some particular examples.

The initial topology of the field lines in the new solutions will determine the evolution of the field, and the value of quantities like the energy, the momentum and the magnetic and electric helicities.

\subsection{Construction of the class}

Let $\phi_{0}(\mathbf{r}), \theta_{0}(\mathbf{r})$, two complex scalar fields that depend on the position $\mathbf{r}$ in the physical space $R^{3}$. We impose that, at some initial time $t=0$, the level curves of the complex scalar fields $\left(\phi_{0}, \theta_{0}\right)$ coincide with the magnetic and 
electric lines, so the magnetic and electric fields at $t=0$ will be

$$
\begin{aligned}
& \mathbf{B}(\mathbf{r}, 0)=\frac{\sqrt{a}}{2 \pi i} \frac{\nabla \phi_{0} \times \nabla \bar{\phi}_{0}}{\left(1+\bar{\phi}_{0} \phi_{0}\right)^{2}}, \\
& \mathbf{E}(\mathbf{r}, 0)=\frac{\sqrt{a} c}{2 \pi i} \frac{\nabla \bar{\theta}_{0} \times \nabla \theta_{0}}{\left(1+\bar{\theta}_{0} \theta_{0}\right)^{2}} .
\end{aligned}
$$

The notation $\bar{\phi}$ means the complex conjugate of $\phi$ and $i$ is the imaginary unit. The expressions (147)-(148) are such that they satisfy $\nabla \cdot \mathbf{B}=0$ and $\nabla \cdot \mathbf{E}=0$ for any scalar fields $\phi$ and $\theta$, which is a necessary condition for $\mathbf{B}(\mathbf{r}, 0)$ and $\mathbf{E}(\mathbf{r}, 0)$ to be admissible initial values of an electromagnetic field in vacuum.

We take both scalar fields $\phi_{0}$ and $\theta_{0}$ as maps $S^{3} \rightarrow S^{2}$ after identifying the physical space $R^{3}$ with $S^{3}$ as we did for the Hopfion, so by construction at $t=0$, all the pairs of field lines of $\mathbf{B}(\mathbf{r}, 0)$ are linked, and the linking number is given by the Hopf index $H\left(\phi_{0}\right)(103)$ of the map $\phi_{0}$, being the same for all pairs of lines. Correspondingly, all pairs of lines of the field $\mathbf{E}(\mathbf{r}, 0)$ are linked, the linking number of all pairs of lines is the same and it is given by the Hopf index $H\left(\theta_{0}\right)$ (104) of the map $\theta_{0}$.

As for the Hopfion case, dimensionless coordinates $(X, Y, Z, T)$, related to the physical ones $(x, y, z, t)$ in the SI of units by $(X, Y, Z, T)=(x, y, z, c t) / L_{0}$, being $c$ the speed of light are introduced. We will also use the relation $r^{2} / L_{0}^{2}=$ $\left(x^{2}+y^{2}+z^{2}\right) / L_{0}^{2}=X^{2}+Y^{2}+Z^{2}=R^{2}$, where $L_{0}$ is a constant with dimensions of length that can be considered to be the characteristic size of the knot.

The complex scalar fields are chosen as,

$$
\begin{gathered}
\phi_{0}=\frac{(X+i Y)^{(n)}}{\left(Z+i\left(R^{2}-1\right) / 2\right)^{(m)}}, \\
\theta_{0}=\frac{(Y+i Z)^{(l)}}{\left(X+i\left(R^{2}-1\right) / 2\right)^{(s)}},
\end{gathered}
$$

where $n, m, l$ and $s$ are positive integer numbers. These scalar fields are related to the Seifert fibrations [64]. The main difference is that, in equations (149) and (150)), the notation $\eta^{(n)}, \eta$ being a complex number, means to leave the modulus of $\eta$ invariant while the phase of $\eta$ is multiplied by $n$.

Level curves of the complex scalar fields given by expression (149) and (150) are $(n, m)$ linked torus knots. If we choose any complex number, say $2+i$, the 


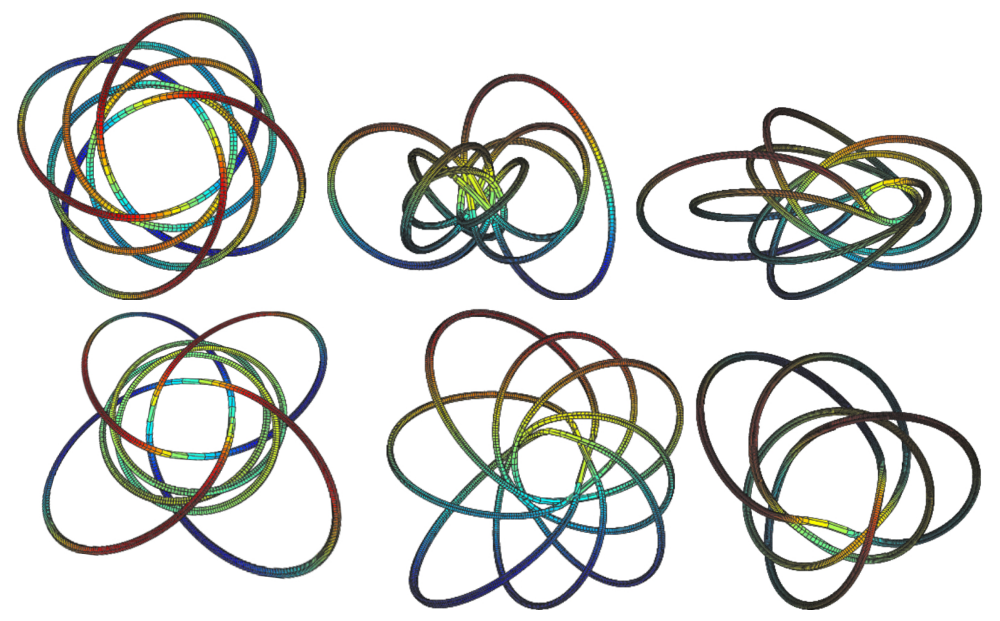

Figure 10: Time evolution of field lines for the $(4,3)$ torus knot. We plot two magnetic (up) and electric (down) lines for $T=0, T=0.015$ and $T=0.02$ (left to right). Lines are obtained numerically by integrating the explicit time dependent expressions (151)-(152) of the magnetic and the electric field. At $T=0$ all the field lines are linked $(4,3)$ torus knots and the linking number is $n m=12$. When $T>0$ we find $(4,3)$ linked torus knots.

equation $\phi=2+i$ gives a curve in the space $R^{3}$, and this curve is a $(n, m)$ torus knot. If we choose any other value, say $1-i$, we get another $(n, m)$ torus knot. The tangent vectors of such curves are parallel to $\nabla \Re(\phi) \times \nabla \Im(\phi)$. Moreover, both curves are linked and their linking number is precisely the Hopf index of $\phi$, that is $H(\phi)=n m$ in this case. This occurs for any level curves of $\phi$. Since level curves of $\phi$ coincide, through equation (147), with magnetic lines at $t=0$, we can say that any pair of magnetic lines at $t=0$ is a linked pair of $(n, m)$ torus knots, and that the linking number is $n m$. The same can be said about the complex scalar field $\theta$ given by equation (150) and the electric field at $t=0$ given by equation (148), so that any pair of electric lines at $t=0$ is a linked pair of $(l, s)$ torus knots and the linking number is $H(\theta)=l s$.

Now, proceeding like in the case of the Hopfion, it was found that the electric 
and magnetic fields are given by [18]

$$
\begin{aligned}
\mathbf{B}(\mathbf{r}, t) & =\frac{\sqrt{a}}{\pi L_{0}^{2}} \frac{Q \mathbf{H}_{1}+P \mathbf{H}_{2}}{\left(A^{2}+T^{2}\right)^{3}} \\
\mathbf{E}(\mathbf{r}, t) & =\frac{\sqrt{a} c}{\pi L_{0}^{2}} \frac{Q \mathbf{H}_{4}-P \mathbf{H}_{3}}{\left(A^{2}+T^{2}\right)^{3}}
\end{aligned}
$$

where the quantities $A, P, Q$ are defined by

$$
\begin{aligned}
& A=\frac{R^{2}-T^{2}+1}{2}, \\
& P=T\left(T^{2}-3 A^{2}\right), \\
& Q=A\left(A^{2}-3 T^{2}\right),
\end{aligned}
$$

and the vectors $\mathbf{H}_{1}, \mathbf{H}_{2}, \mathbf{H}_{3}$ and $\mathbf{H}_{4}$ are

$$
\begin{aligned}
\mathbf{H}_{1}= & (-n X Z+m Y+s T) \mathbf{u}_{x}+(-n Y Z-m X-l T Z) \mathbf{u}_{y} \\
& +\left(n \frac{-1-Z^{2}+X^{2}+Y^{2}+T^{2}}{2}+l T Y\right) \mathbf{u}_{z}, \\
\mathbf{H}_{2}= & \left(s \frac{1+X^{2}-Y^{2}-Z^{2}-T^{2}}{2}-m T Y\right) \mathbf{u}_{x} \\
& +(s X Y-l Z+m T X) \mathbf{u}_{y}+(s X Z+l Y+n T) \mathbf{u}_{z}, \\
\mathbf{H}_{3}= & (-m X Z+n Y+l T) \mathbf{u}_{x}+(-m Y Z-n X-s T Z) \mathbf{u}_{y} \\
& +\left(m \frac{-1-Z^{2}+X^{2}+Y^{2}+T^{2}}{2}+s T Y\right) \mathbf{u}_{z}, \\
\mathbf{H}_{4}= & \left(l \frac{1+X^{2}-Y^{2}-Z^{2}-T^{2}}{2}-n T Y\right) \mathbf{u}_{x} \\
& +(l X Y-s Z+n T X) \mathbf{u}_{y}+(l X Z+s Y+m T) \mathbf{u}_{z} .
\end{aligned}
$$

Using the expressions (151) and (152), the field lines at any instant of time can be numerically computed. We have plotted few lines for the case $(n, m, l, s)=(4,3,4,3)$ in Fig. 10. At $T=0$, all the lines are linked $(4,3)$ torus knots and the linking number is $n m=12$. These are the only kind of curves that can be found since these lines are level curves of the scalar fields (149)(150). For $T>0$ we find lines with the same topology. However, as $T$ increases, numerical evidence prevent us to rule out the existence of open field lines. A similar behaviour was found also numerically for knot-like fields constructed from Chandrasekhar-Kendall curl eigenstates [17]. 


\subsection{Properties of the class}

800 ants are

$$
\begin{aligned}
\mathbf{E} \cdot \mathbf{B}= & \frac{a c}{\pi^{2} L_{0}^{4}} \frac{1}{\left(A^{2}+T^{2}\right)^{4}}\left[(m s-n l)\left(A^{2}+T^{2}\right)\right. \\
& +(m l-n s)\left(A^{2}+T^{2}\right)(1-A) Y \\
& \left.+2(l s-m n) A T\left(T^{2}-A^{2}\right)\right], \\
E^{2}-c^{2} B^{2}= & \frac{a c^{2}}{\pi^{2} L_{0}^{4}} \frac{1}{\left(A^{2}+T^{2}\right)^{4}}\left[\left(n^{2}-m^{2}\right)\left(A^{2}+T^{2}\right)\left(X^{2}+Y^{2}\right)\right. \\
& +\left(s^{2}-l^{2}\right)\left(A^{2}+T^{2}\right)\left(Y^{2}+Z^{2}\right)+4\left(m^{2}-s^{2}\right) A^{2} T^{2} \\
& \left.-\left(n^{2}-l^{2}\right)\left(A^{2}-T^{2}\right)^{2}\right] .
\end{aligned}
$$

The particular choice of the set $(n, m, l, s)$ determines the properties of the new torus knots class:

- If the positive integer numbers $n, m, l, s$ are equal, i.e. satisfy the condition $n=m=l=s$, then $\mathbf{E} \cdot \mathbf{B}=0$ and $E^{2}-c^{2} B^{2}=0$. This is the case of the Hopfion.

- If the condition $n=m=l=s$ is not fulfilled, then $\mathbf{E} \cdot \mathbf{B} \neq 0$ and $E^{2}-c^{2} B^{2} \neq 0$. We have a non-null electromagnetic field in vacuum. This field is non-null and non-decomposable.

With the help of equation (157), the time behaviour of the helicities of these electromagnetic fields can be exactly computed. The calculations yield

$$
\begin{aligned}
h_{m}(t) & =\frac{1}{2 \mu_{0}} \int d^{3} r \mathbf{A} \cdot \mathbf{B} \\
& =\frac{a}{4 \mu_{0}}\left[(m n+l s)+(m n-l s) \frac{1-6 T^{2}+T^{4}}{\left(1+T^{2}\right)^{4}}\right], \\
h_{e}(t) & =\frac{\varepsilon_{0}}{2} \int d^{3} r \mathbf{C} \cdot \mathbf{E} \\
& =\frac{a}{4 \mu_{0}}\left[(m n+l s)-(m n-l s) \frac{1-6 T^{2}+T^{4}}{\left(1+T^{2}\right)^{4}}\right],
\end{aligned}
$$

where we recall that $T=c t / L_{0}$, being $t$ the time. 


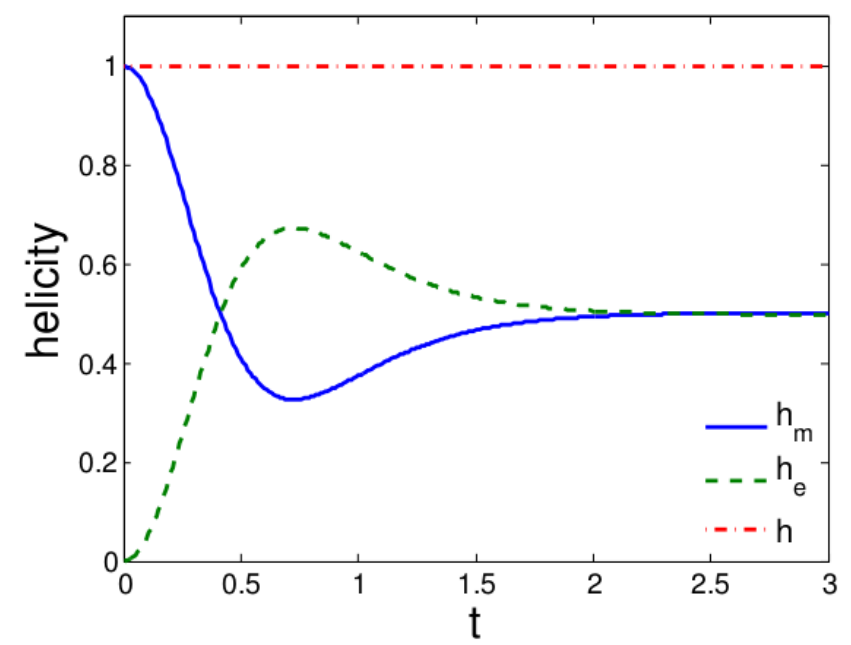

Figure 11: (Colour online) Time evolution of the magnetic $h_{m}$ (blue solid line) and electric $h_{e}$ (green dashed line) helicities in units of $a /\left(2 \mu_{0}\right)$. Initially, fot $t=0, h_{m}=1$ and $h_{e}=0$. The sum of both is the electromagnetic helicity (red dash-dot line), that is equal to 1 in the same units. The interchange between magnetic and electric helicities is clear in the figure. Both helicities reach a common value for large values of time.

If, at $t=0$, we have $h_{m} \neq h_{e}$, then both helicities are not constant but, for large time, both reach the same value. This property was first discovered and studied in [36], and called the exchange of helicities mechanism, as the total electromagnetic helicity

$$
h_{e m}=h_{m}(t)+h_{e}(t)=\frac{a}{2 \mu_{0}}(n m+l s),
$$

remains constant. An explicit example of the interchange of helicities is plotted in Fig. 11 in which $n=m=1$ and $l=s=0$. When $t$ is large, both helicities coincide, i. e. $\lim _{t \rightarrow \infty} h_{m}(t)=\lim _{t \rightarrow \infty} h_{e}(t)$. This result suggests that there is an exchange of helicity between the electric and magnetic field until they reaches a common value.

Another important property is that the electromagnetic energy content of the fields remains finite and is related to the square of the integer numbers 


$$
\begin{aligned}
& (m, n, s, l) \text { by } \\
& \qquad \mathcal{E}=\int d^{3} r\left(\frac{\varepsilon_{0} E^{2}}{2}+\frac{B^{2}}{2 \mu_{0}}\right)=\frac{a}{2 \mu_{0} L_{0}}\left(n^{2}+m^{2}+l^{2}+s^{2}\right) .
\end{aligned}
$$

The linear momentum of these knotted solutions can also be obtained from the Poynting vector $\mathbf{E} \times \mathbf{B} / \mu_{0}$ and results

$$
\mathbf{p}=\int \varepsilon_{0} \mathbf{E} \times \mathbf{B}=\frac{a}{2 c \mu_{0} L_{0}}(\ln +m s) \mathbf{u}_{y} .
$$

In general, using vector identities it is easily proved that

$$
\left|\int d^{3} r \varepsilon_{0} \mathbf{E} \times \mathbf{B}\right| \leq \frac{1}{c} \int d^{3} r\left(\frac{\varepsilon_{0} E^{2}}{2}+\frac{B^{2}}{2 \mu_{0}}\right),
$$

The equality holds only when the field transport energy at the speed of light (see [65]), being the velocity at which the energy is transported defined as the time derivative of

$$
<\mathbf{r}>=\frac{\int \mathbf{r} U d^{3} r}{\int U d^{3} r}
$$

i.e. the equality for expression (163) is obtained when

$$
v_{u}=\left|\frac{d<\mathbf{r}>}{d t}\right|=c .
$$

However, from expressions (161) and (162), we always have the strict inequality

$$
p<\frac{\mathcal{E}}{c},
$$

being $p=|\mathbf{p}|$. Thus we can attribute a rest energy to our electromagnetic field given by the relation

$$
\mathcal{E}^{2}=p^{2} c^{2}+\mathcal{E}_{0}^{2} .
$$

Taking into account results (161) and (162) for the energy and linear momentum of these knotted electromagnetic structures, $\mathcal{E}_{0}$ reads

$$
\mathcal{E}_{0}=\frac{a}{2 \mu_{0} L_{0}} \sqrt{\left(n^{2}+m^{2}+l^{2}+s^{2}\right)^{2}-(l n+m s)^{2}} .
$$

This rest energy depends on the characteristic size of the knot $L_{0}$, on the constant $a$ included in the definition of this kind of knotted electromagnetic structures (147)-(148) and on the integer numbers $(n, m, l, s)$ which determine the topological nature of the magnetic and electric torus knots. 

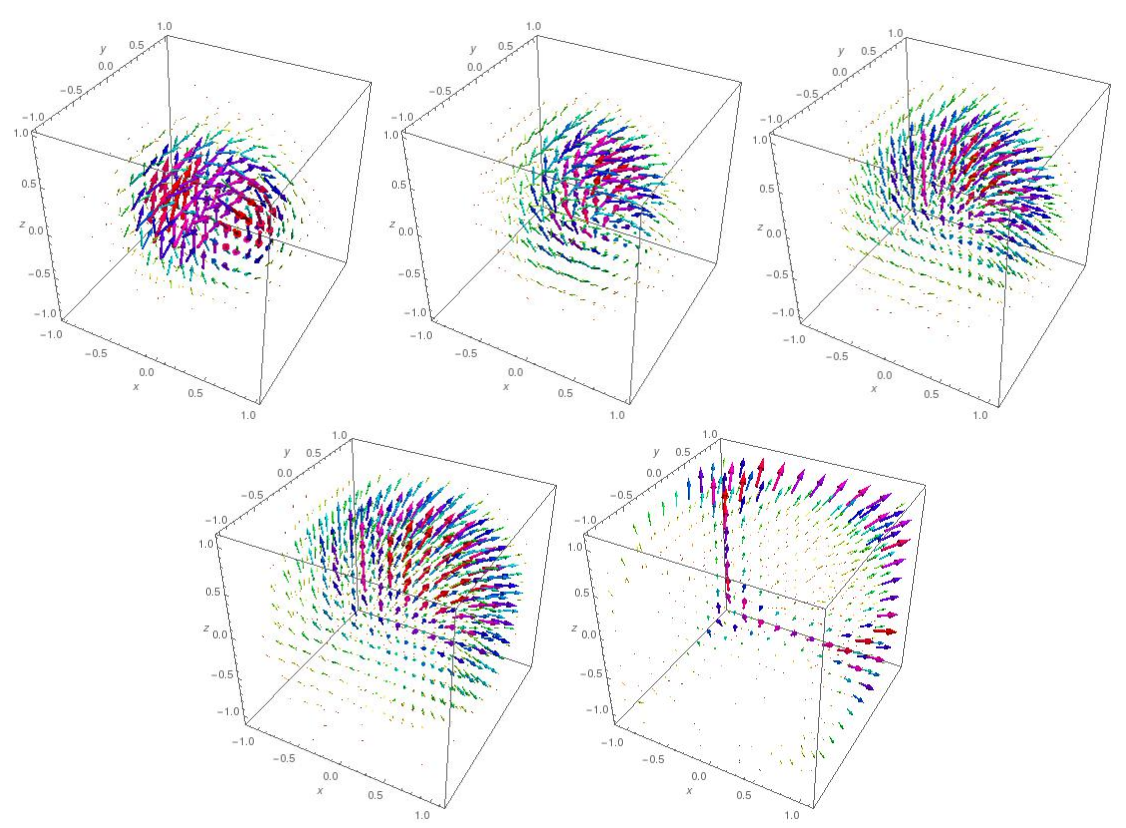

Figure 12: Time evolution of the Poynting vector $\mathbf{E} \times \mathbf{B} / \mu_{0}$ for the electromagnetic field given by equations (151)-(152) in the case in which $n=2, m=3, s=2, l=3$. We plot the Poynting vectors at some points of space for $T=0, T=0.5, T=1, T=1.5$ and $T=2.5$ (left to right, up to bottom).

A way to picture the evolution of these fields is to plot Poynting vectors at some points in space at different times. In Fig. 12, we see the behaviour of these electromagnetic fields for instants of time $T=0,0.5,1,1.5,2.5$. In the figure we have plotted the case $(n, m, s, l)=(2,3,2,3)$ for the initial magnetic field lines, and $(l, s)=(2,3)$ for the initial electric field lines. The magnetic and electric fields are given by equations (151) and (152) with $n=3, m=4, l=2, s=3$.

\section{Null torus knotted electromagnetic fields}

We have discussed how to build electromagnetic fields in vacuum covering the whole torus set of knots in at least one instant of time. At that particular time they are globally described by two Rañada's scalar fields $\phi$ and $\theta$. These solutions were non-null fields. 
In this section we present a new class of solutions [19]. If the null condition is preserved during time evolution, the electric and magnetic field remain perpendicular which impose a strong geometric constrain on the topology of the field lines. By Robinson's theorem [62], there is a shear-free family of light rays, underlying each null electromagnetic field. The light rays are given by the Poynting vector, and the electromagnetic field evolution can be thought of as a fluid-like, being transported along the world lines of the light rays. The rays follow geodesics paths in free space. The angles between $\mathbf{E}$ and $\mathbf{B}$ are preserved along those paths as they remain perpendicular, so the fluid evolution is shearfree. The null condition imposes then a strong constrain on the configuration of the field lines, reducing the problem to find a triplet of mutually orthogonal fields that remain orthogonal under time evolution. It turns out that it is possible to build null solutions whose field lines also encode torus knots and links [19].

Bateman [20] developed a formalism for the construction of the null fields with the introduction of complex Euler potentials instead of real ones. Also one can use the spinor formalism to achieve the same results [12]. We will briefly review here the Bateman's construction, leaving the spinor formulation for a latter section.

\subsection{Bateman's null fields}

Bateman construction [20] is based on the introduction of two complex scalar fields $\alpha$ and $\beta$ which are functions of space-time coordinates. In order that they generate electromagnetic fields, they have to fulfil the conditions

$$
\begin{aligned}
\partial_{\mu} \alpha \partial^{\mu} \alpha & =0, \\
\partial_{\mu} \beta \partial^{\mu} \beta & =0, \\
\partial_{\mu} \alpha \partial^{\mu} \beta & =0 .
\end{aligned}
$$

In the original work, Bateman introduced the complex vector [20] $\mathbf{M}=$ $c \mathbf{B} \pm i \mathbf{E}$, so Maxwell's equation in vacuum (3) and (4) can be written as

$$
\nabla \times \mathbf{M}=\mp \frac{i}{c} \frac{\partial \mathbf{M}}{\partial t}, \nabla \cdot \mathbf{M}=0 .
$$


Note that Silberstein [66] made use of the same vector without the \pm sign, only choosing one sign and defining $c \mathbf{B}-i \mathbf{E}$ and its complex conjugate. Earlier, according to Weber [67], Riemann originally took $\mathbf{F}=\mathbf{E}+i c \mathbf{B}$. Some authors call this last one the Riemann-Silbertein vector $\mathbf{F}$ and we will follow this convention [68].

If we can find two functions $(\alpha, \beta)$ such that the conditions (169) are satisfied, we can write

$$
\mathbf{M}=\nabla \alpha \times \nabla \beta
$$

as a solution to the equations (170). The scalar functions $(\alpha, \beta)$ play the same role as the Euler potentials but they are now complex functions. We can check that by construction we get null fields as $\mathbf{M}^{2}=0$.

\subsection{Knotted fields based on Bateman's construction}

We have already discussed the Hopf-Rañada solution or Hopfion and how it can be expressed in terms of Euler potentials. It was found that the Hopfion solution also can be written in terms of the two complex scalar fields $(\alpha, \beta)$ using Bateman's contruction [61], as the Hopfion is a null electromagnetic field.

The Riemann-Silberstein complex vector for the Hopfion is easily calculated from (116) as

$$
\mathbf{F}(\mathbf{r}, t)=\frac{\sqrt{a} c}{\pi L_{0}^{2}(A+i T)^{3}}\left[\begin{array}{c}
\frac{1+X^{2}-Z^{2}-(Y+T)^{2}}{2}+i(Y+T-X Z) \\
(X-i Z)(Y+T-i) \\
Y+T+X Z+i\left(\frac{-1+X^{2}-Z^{2}+(Y+T)^{2}}{2}\right)
\end{array}\right] .
$$

We recall that $A$ is given by (115). It can be easily checked that the Riemann vector can be written as

$$
\mathbf{F}(\mathbf{r}, t)=\frac{c \sqrt{a}}{2 \pi L_{0}^{2}} \nabla \alpha_{h} \times \nabla \beta_{h}
$$

with the complex potentials

$$
\begin{aligned}
\alpha_{h} & =-\frac{2 Z+2 X i}{Y+T-i} \\
\beta_{h} & =\left(\frac{Y+T-i}{X^{2}+Y^{2}+Z^{2}-(T-i)^{2}}\right)^{2} .
\end{aligned}
$$


Using Bateman's framework, and the fact that there is a natural connection between knots and singular points of complex maps [69], a new class of knotted solutions was found with the property that there is at least a singular line with the topology of a torus knot and they are null fields [19]. In particular, the intersection of the four dimensional sphere $S^{3}$ with the complex hypersurface ${ }_{875} z_{1}^{n} \pm z_{2}^{m}=0$ (where $z_{1}, z_{2} \in C$ ), gives a $(n, m)$ torus knot when $n$ and $m$ are coprime integers. This fact was used to construct knotted optical vortices in light beams [70]. The trick is to take the following Bateman pair $(\alpha, \beta)$ based on the stereographic projection of $S^{3}$,

$$
\begin{aligned}
\alpha & =\frac{X^{2}+Y^{2}+Z^{2}-T^{2}-1+2 Z i}{X^{2}+Y^{2}+Z^{2}-(T-i)^{2}}, \\
\beta & =\frac{2 X-2 Y i}{X^{2}+Y^{2}+Z^{2}-(T-i)^{2}},
\end{aligned}
$$

so $|\alpha|^{2}+|\beta|^{2}=1$, and $\alpha^{n} \pm \beta^{m}=0$ gives torus knot lines if as said before $n$ and $m$ are coprime. The potentials are taken as $\alpha^{n}$ and $\beta^{m}$, and consequently the fields can be obtained using the Riemann vector as before

$$
\mathbf{F}=\nabla \alpha^{n} \times \nabla \beta^{m}
$$

with $(\alpha, \beta)$ given by (176).

Specifically, the magnetic lines are organised around a set of core field lines which are closed and form $(n, m)$ torus knots or links when $n$ and $m$ are coprimes, as plotted in Fig. 9. Again, as in the case of the class of non-null torus fields, the Hopfion is contained in this class for the particular values $n=m=1$.

The magnetic and electric helicities are equal for this new class. Normalised by the energy, and in natural units, their values are [19]

$$
\frac{h_{e}}{\mathcal{E}}=\frac{h_{m}}{\mathcal{E}}=\frac{1}{n+m} .
$$

The linear momentum turns out to be in the $z$-direction, and normalized by the energy reads

$$
\frac{\mathbf{p}}{\mathcal{E}}=\left(0,0, \frac{-n}{n+m}\right) .
$$

For more details of the properties of those solutions, we refer to [19]. 


\section{Conformal transformations}

There are several treads in the literature based on introducing complex space-time values in order to transform a familiar solution (e.g. of the Maxwell equations or Einstein equations) into a new solution. Two well know examples are the construction of Gaussian optical beams propagating in, say, the $z$ direction, from the field of a point charge at a location on the imaginary $z$ axis, and the construction of the Kerr spacetime for a rotating black hole from the Schwarzchild spacetime solution via a shift of spacetime variables into the complex plane. This method is often combined with a change of the coordinate system, as is the case for the Kerr solution where cartesian coordinates are replaced by oblate spherical coordinates. We will show that this technique can transform trivial solutions of the Maxwell equations, as simple as uniform and orthogonal $\mathbf{E}$ and $\mathbf{B}$ fields, into the topologically nontrivial solutions discussed in the previous sections.

The method of considering complex shifts dates back at least to the year 1887 in which P. Appell wrote a remarkable letter to F. Klein. In order to familiarize ourselves with this concept we will briefly review this letter, as well as the constructions of Gaussian beams and the Kerr solution before addressing linked and knotted light field. Then we follow the construction given in [29] to obtain new fields from old ones using conformal inversion and complex shifts. This method is further generalized in [21].

\subsection{Letter from Appell}

In a letter from P. Appell to F. Klein [71] solutions to Laplace's equation $\Delta \psi=0$ are considered. In 3 dimensions,

$$
\psi(x, y, z)=\frac{A}{\sqrt{(x-\alpha)^{2}+(y-\beta)^{2}+\left(z-\sigma^{2}\right)}}
$$

is a solution which is singular at $(\alpha, \beta, \gamma)$ for $\alpha, \beta, \gamma$ real. Appell considered solutions with complex values: $A=\lambda+i \mu, \alpha=a+i a^{\prime}, \beta=b+i b^{\prime}$, and $\gamma=c+i c^{\prime}$, with $\lambda, \mu, a, a^{\prime}, b, b^{\prime}, c, c^{\prime}$, real. A real field $\psi(x, y, z)$ can still 
be obtained by simply adding the complex conjugate (which is also a solution).

This gives

$$
\psi(x, y, z)=\frac{A}{\sqrt{S-i P}}+\frac{\bar{A}}{\sqrt{S+i P}}
$$

with

$$
S=(x-a)^{2}+(y-b)^{2}+(z-c)^{2}-a^{\prime 2}-b^{\prime 2}-c^{\prime 2},
$$

and

$$
P=2 a^{\prime}(x-a)+2 b^{\prime}(y-b)+2 c^{\prime}(z-c) .
$$

$S=0$ defines a sphere with radius $a^{\prime 2}+b^{\prime 2}+c^{\prime 2}$ centered around $(a, b, c)$, and $P=0$ is a plane through the center of the sphere $S=0$. This means that

$\psi(x, y, z)$ is singular on a circle (the intersection of $S=0$ and $P=0$ ). There is something very peculiar about this situation. The fact that we now deal with a singular circle implies that there is the possibility of following the field along a loop that is linked with the singular ring. Since $P$ changes sign when crossing the plane $P=0$ and $S$ changes sign when crossing the surface of the sphere $S=0$ the loop is topologically equivalent to a circle around the origin in the complex $(S, i P)$ plane. Since the denominators of the two terms in (181) are complex square roots $\sqrt{z}=\sqrt{r} e^{i \theta / 2}$ they pick up a minus sign by completing a loop $(\theta=0$ to $\theta=2 \pi)$ around the origin. This two-valuedness of $\psi(x, y, z)$ is of course the result of the Riemann surface of the complex square root. Still, by construction, we are considering only real valued solutions $\psi(x, y, x)$ to the Laplace equation.

\subsection{Gaussian beams}

In 1971 Deschamps showed that a Gaussian beam is equivalent in paraxial approximation to a spherical wave with a center at a complex location [30]. Consider the wave equation for

$$
\frac{1}{c^{2}} \frac{\partial^{2}}{\partial^{2} t} \psi(x, y, z, t)-\nabla^{2} \psi(x, y, z, t)=0
$$

For a monochromatic solution with $\omega / c=k$ the equation for the spatial part of the wave function reduces to $\left(\nabla^{2}+k^{2}\right) \psi(x, y, z)=0$. The radial function

$$
\psi(x, y, z)=\frac{e^{i k r}}{r}
$$


with $r$ the distance of the point $(x, y, z)$ from the origin, is a solution to the wave equation.

We now want to consider beam like solutions along the $z$-axis such that the amplitude variations in $x, y$ directions are much larger than in the $z$ direction. Therefore the second order derivative with respect to $z$ can be neglected and the approximate paraxial wave equation becomes

$$
\frac{1}{i} \frac{\partial}{\partial z}=k-\frac{1}{2 k}\left(\frac{\partial^{2}}{\partial x^{2}}+\frac{\partial^{2}}{\partial y^{2}}\right) .
$$

Deschamps noticed that by taking the solution in (182) such that the origin is $(0,0, i a)$ and assuming that $|x|$ and $|y|$ are much smaller than $|z-i a|$ the solution

represent a Gaussian beam near the $z$-axis. This is seen by approximating $r$ in the exponent as

$$
r=\sqrt{(z-i a)^{2}+x^{2}+y^{2}} \approx z-i a+\frac{1}{2} \frac{x^{2}+y^{2}}{z-i a}
$$

and $r \approx z-i a$ in the denominator. The approximate solution then reads

$$
\psi(x, y, z)=\frac{e^{i k(z-i a)}}{z-i a} \exp \left(\frac{i k}{2} \frac{x^{2}+y^{2}}{z-i a}\right) .
$$

It is easily verified that (184) is indeed a Gaussian beam solution of the paraxial 925 wave equation (183). Since lasers produce such beams to a good approximation this solution is highly relevant for practical applications.

\subsection{Kerr solution}

In Cartesian coordinates, the Schwarzschild geometry in Kerr-Schild form $[72]$ is

$$
g_{a b}=\eta_{a b}+\frac{2 m}{r} l_{a} l_{b}
$$

where $\eta_{a b}=<-1,+1,+1,+1>$ and $l_{a}=(1, x / r, y / r, z / r)$, with $r$ the radial distance from the origin to the point $(x, y, z)$.

The Kerr solution for a rotating black hole is now obtained from the Schwarzschild solution by first considering $(r, \theta, \psi)$ to be oblate spheroidal coordinates, such that,

$$
x+i y=(r+i a) \sin \theta e^{i \psi}, \quad z=r \cos \theta
$$


and second, by replacing $2 m / r$ by

$$
m\left(\frac{1}{r+i a \cos \theta}+\frac{1}{r-i a \cos \theta}\right)=\frac{2 m r}{r^{2}+a^{2} \cos ^{2} \theta} .
$$

930 shift of the parameter $r$ and adding the complex conjugate to obtain a real valued expression, and in the first step we see a coordinate transformation. This transformation is obviously not a Poincaré transformation and therefore does not simply represent a transformation of a solution to a different inertial $\mathcal{C}(1,3)$ for a spin 1 vector field uniquely leads to source-free Maxwell equations [75].

When considering Poincaré transformations of electromagnetic fields, we do not generate new solutions of the Maxwell equations but rather describe the same solution in a different inertial reference frame. However when one considers special conformal transformations of an electromagnetic field one does obtain genuine new solutions of the Maxwell equations.

Every element of the conformal group that does not belong to the Poincaré group (dilation and four special conformal transformations) can be composed from Poincaré transformations and conformal inversions, which, like time reversal, parity and charge conjugation, form discrete symmetry operations.

A special conformal transformation $\mathcal{S C T}(b)$ is given by

$$
x^{\mu} \rightarrow \tilde{x}^{\mu}=\frac{x^{\mu}+b^{\mu}\left(x_{\nu} x^{\nu}\right)}{1+2\left(b_{\nu} x^{\nu}\right)+\left(x_{\nu} x^{\nu}\right)\left(b_{\nu} b^{\nu}\right)},
$$

and can be built from a conformal inversion $\mathcal{C} \mathcal{I}(a)$,

$$
x^{\mu} \rightarrow \tilde{x}^{\mu}=a \frac{x^{\mu}}{x_{\nu} x^{\nu}},
$$


and a translation $\mathcal{T}(c)$,

$$
x^{\mu} \rightarrow \tilde{x}^{\mu}=x^{\mu}+c^{\mu},
$$

as follows

$$
\mathcal{S C T}(b)=\mathcal{C I}(1) \circ \mathcal{T}(b) \circ \mathcal{C I}(1) .
$$

Dilations $\mathcal{D}(a)$,

$$
x^{\mu} \rightarrow a x^{\mu},
$$

can be obtained in the same way, but with different parameters,

$$
\mathcal{D}(a)=\mathcal{C I}(a) \circ \mathcal{T}(0) \circ \mathcal{C I}(1)=\mathcal{C} \mathcal{I}(a) \circ \mathcal{C I}(1) .
$$

Since any conformal transformation can be built from Poincaré transformations and conformal inversions, we are primarily interested in the role of conformal inversion in generating new solutions from existing solutions of the Maxwell equations. Note that a conformal inversion (187) is singular for points on the light-cone. A general discussion of the conformal group should therefore include the compactification of Minkowski space [76]. However, for the following discussion it will suffice to consider the Minkowski space.

In order to arrive at new solutions from existing ones, we can proceed as follows [29]. Let us have two Minkowski spaces with coordinates $x^{\mu} \in M^{4}$ and $\tilde{x}^{\mu} \in \tilde{M}^{4}$, and a mapping between these manifolds given by a conformal inversion $\mathcal{C} \mathcal{I}(1)(187)$,

$$
f: x^{\mu} \rightarrow \tilde{x}^{\mu}(x)=\frac{x^{\mu}}{x \cdot x} .
$$

Here we use the notation $x \cdot x$ for $x_{\nu} x^{\nu}$. For the inverse we get

$$
f^{-1}: \tilde{x}^{\mu} \rightarrow x^{\mu}(\tilde{x})=\frac{\tilde{x}^{\mu}}{(\tilde{x} \cdot \tilde{x})} .
$$

We use $f$ to pull back tensor fields to $M^{4}$. In this way the metric tensor $\tilde{\eta}_{\mu \nu}$ is mapped to

$$
g_{\mu \nu}(x)=\left(f^{p b} \tilde{\eta}\right)_{\mu \nu}(x)=\frac{\partial \tilde{x}^{\alpha}(x)}{\partial x^{\mu}} \frac{\partial \tilde{x}^{\beta}(x)}{\partial x^{\nu}} \tilde{\eta}_{\alpha \beta}=\frac{1}{(x \cdot x)^{2}} \eta_{\mu \nu}
$$

as $\tilde{\eta}_{\mu \nu}=\eta_{\mu \nu}$ and this shows that, by definition, $f$ is a conformal map [46]. We will regard tensor fields in $M^{4}$ and therefore use $\eta_{\mu \nu}$ to lower or raise indices, 
not $g_{\mu \nu}$. We proceed with pulling back a Faraday or electromagnetic field tensor satisfying source-free Maxwell equations $\left(\tilde{F}_{\mu \nu}\right)$ from $\tilde{M}^{4}$,

$$
F_{\mu \nu}(x)=\left(f^{p b} \tilde{F}\right)_{\mu \nu}(x)=\frac{\partial \tilde{x}^{\alpha}(x)}{\partial x^{\mu}} \frac{\partial \tilde{x}^{\beta}(x)}{\partial x^{\nu}} \tilde{F}_{\alpha \beta}(\tilde{x}(x)),
$$

and

$$
F^{\mu \nu}(x)=\eta^{\mu \alpha} \eta^{\nu \beta} F_{\alpha \beta}=\eta^{\mu \alpha} \eta^{\nu \beta} \frac{\partial \tilde{x}^{\sigma}}{\partial x^{\alpha}} \frac{\partial \tilde{x}^{\kappa}}{\partial x^{\beta}} \eta_{\sigma \lambda} \eta_{\kappa \rho} \tilde{F}^{\lambda \rho}(\tilde{x}(x))=\left|\frac{\partial \tilde{x}}{\partial x}\right| \frac{\partial x^{\mu}}{\partial \tilde{x}^{\lambda}} \frac{\partial x^{\nu}}{\partial \tilde{x}^{\rho}} \tilde{F}^{\lambda \rho},
$$

in which we introduced the absolute value of the Jacobian in the last step, being $|\partial \tilde{x} / \partial x|=1 /(x \cdot x)^{4}$. These are tensor fields on $M^{4}$. Since $\tilde{F}$ is a Faraday tensor on $\tilde{M}^{4}, F$ is expected to be also a Faraday tensor on $M^{4}$, satisfying the Biachi identity (10). From (190),

$$
\partial_{\lambda} F_{\mu \nu}(x)+\partial_{\nu} F_{\lambda \mu}(x)+\partial_{\mu} F_{\nu \lambda}(x)=0,
$$

follows by reshuffling of indices, systematic cancellation of terms and using the fact that $\tilde{F}$ is a Faraday tensor on $\tilde{M}^{4}$. Getting the dynamical equations (11) is more intricate, but again from (191),

$$
\partial_{\mu} F^{\mu \nu}(x)=0 .
$$

With this result we end up with a prescription for constructing new vacuum solutions to the Maxwell equations from known ones: in $M^{4}$ we can make an conformal inverse transform of coordinates. If $F$ is a Faraday tensor then $F^{\prime}$ is also a Faraday tensor, in which

$$
F_{\mu \nu}^{\prime}(x)=\left(\partial_{\mu} f^{\alpha}\right)\left(\partial_{\nu} f^{\beta}\right) F_{\alpha \beta}(\tilde{x}(x))
$$

and

$$
F^{\prime \mu \nu}(x)=\eta^{\mu \alpha} \eta^{\nu \beta} F_{\alpha \beta}^{\prime}(x) .
$$

As pointerd out in Section 6 it can be advantageous to use the RiemannSilberstein (RS) vector $\mathbf{F}=\mathbf{E}+i \mathbf{B}$. Instead of the Faraday tensor, $F_{\mu \nu}$, we should then use the self-dual or anti-self-dual form, $F_{\mu \nu}^{(s d)}$ or $F_{\mu \nu}^{(a s d)}$, of it,

$$
\begin{aligned}
F_{\mu \nu}^{(a s d)} & =F_{\mu \nu}+i^{*} F_{\mu \nu} \\
F_{\mu \nu}^{(s d)} & =F_{\mu \nu}-i^{*} F_{\mu \nu}
\end{aligned}
$$


where ${ }^{*} F_{\mu \nu}$ is defined in (15). We can get the complex conjugate of the RS vector $\overline{\mathbf{F}}=\mathbf{E}-i \mathbf{B}$ from the relation

$$
F_{0 i}^{(a s d)}=\overline{\mathbf{F}}_{i}
$$

From the different transformations of $F_{\mu \nu}(190)$ and $F^{\mu \nu}$ it is not immediately clear how to calculate $F^{(a s d) \prime}$ from $F^{(a s d)}$. But in the case of conformal inversions, we have some special properties like

$$
\frac{\partial \tilde{x}^{\nu}}{\partial x^{\mu}}=\left|\frac{\partial \tilde{x}}{\partial x}\right|^{\frac{1}{2}} \frac{\partial x^{\nu}}{\partial \tilde{x}^{\mu}}=\frac{\partial \tilde{x}_{\mu}}{\partial x_{\nu}},
$$

so we get using (191),

$$
F^{\prime \mu \nu}(x)=\left|\frac{\partial \tilde{x}}{\partial x}\right| \frac{\partial x^{\mu}}{\partial \tilde{x}^{\lambda}} \frac{\partial x^{\nu}}{\partial \tilde{x}^{\rho}} F^{\lambda \rho}(\tilde{x}(x))=\frac{\partial \tilde{x}^{\mu}}{\partial x^{\lambda}} \frac{\partial \tilde{x}^{\nu}}{\partial x^{\rho}} F^{\lambda \rho}(\tilde{x}(x)),
$$

and this leads using (190) to

$$
F_{\mu \nu}^{(a s d)^{\prime}}(x)=\frac{\partial \tilde{x}^{\alpha}}{\partial x^{\mu}} \frac{\partial \tilde{x}^{\beta}}{\partial x^{\nu}} F_{\alpha \beta}^{(s d)}(\tilde{x}(x)) .
$$

Thus the self-dual is transformed into an anti-self-dual form. Also

$$
\begin{aligned}
F_{(a s d)}^{\prime \rho \sigma}(x) & =\eta^{\rho \mu} \eta^{\sigma \nu} F_{\mu \nu}^{(a s d) \prime}=\frac{\partial \tilde{x}_{\alpha}}{\partial x_{\rho}} \frac{\partial \tilde{x}_{\beta}}{\partial x_{\sigma}} F_{(s d)}^{\alpha \beta}=\frac{\partial \tilde{x}^{\rho}}{\partial x^{\alpha}} \frac{\partial \tilde{x}^{\sigma}}{\partial x^{\beta}} F_{(s d)}^{\alpha \beta} \\
& =\left|\frac{\partial \tilde{x}}{\partial x}\right| \frac{\partial x^{\rho}}{\partial \tilde{x}^{\alpha}} \frac{\partial x^{\sigma}}{\partial \tilde{x}^{\beta}} F_{(s d)}^{\alpha \beta}(\tilde{x}(x)) .
\end{aligned}
$$

From (197) and (196) the transformation of the RS vector follows [29],

$$
\mathbf{F}^{\prime}(x)=\frac{1}{\left(t^{2}-r^{2}\right)^{3}}\left[-\left(t^{2}-r^{2}\right) \overline{\mathbf{F}}(\tilde{x}(x))+2 \mathbf{r} \times(\mathbf{r} \times \overline{\mathbf{F}}(\tilde{x}(x))-2 i t \mathbf{r} \times \overline{\mathbf{F}}(\tilde{x}(x))] .\right.
$$

where we have used $x=\left(x^{\mu}\right)=(t, \mathbf{r})$. This expression can be used to obtain the transformation of the Poynting vector $\mathbf{P}=\frac{i}{2} \mathbf{F} \times \overline{\mathbf{F}}$ and the energy density $U=\frac{1}{2} \mathbf{F} \cdot \overline{\mathbf{F}}$.

7.5. The Hopf-Rañada field from a conformal transformation of a constant electric and magnetic field

Consider the following Riemann-Silberstein vector

$$
\mathbf{F}=\mathbf{u}_{x}+i \mathbf{u}_{y}=\left(\begin{array}{l}
1 \\
i \\
0
\end{array}\right),
$$


representing a constant $\mathbf{E}$ field in the $x$ direction and a constant $\mathbf{B}$ field in the $y$ direction. Performing conformal inversion (198) to this simple $\mathbf{F}$ gives (taking $c=1$ and $(t, x, y, z)$ dimensionless variables)

$$
\mathbf{F}^{\prime}=\frac{1}{\left(t^{2}-r^{2}\right)^{3}}\left(\begin{array}{c}
(x-i y)^{2}-(t-z)^{2} \\
i(x-i y)^{2}+i(t-z)^{2} \\
-2(x-i y)(t-z)
\end{array}\right)
$$

or explicitly in $\mathbf{E}$ and $\mathbf{B}$ fields

$$
\mathbf{E}^{\prime}=\frac{1}{\left(t^{2}-r^{2}\right)^{3}}\left(\begin{array}{c}
x^{2}-y^{2}-(z-t)^{2} \\
2 y x \\
-2 x(t-z)
\end{array}\right), \quad \mathbf{B}^{\prime}=\frac{1}{\left(t^{2}-r^{2}\right)^{3}}\left(\begin{array}{c}
-2 x y \\
x^{2}-y^{2}+(t-z)^{2} \\
2 y(t-z)
\end{array}\right) .
$$

These fields represent sets of field lines that at any time fill the surface of nested degenerate tori: degenerate in the sense that the central hole is reduced to a singular point were all the fields lines touch. This singular point is the origin at $t=0$ and it then moves along the $z$ axis with the speed of light. Fig. 13 illustrates one of the tori for $\mathbf{E}, \mathbf{B}$, and $\mathbf{P}$, at $t=0$ and at $t=1$.

Now apply the imaginary time translation $t \rightarrow t-i$ (as in Appell's letter) to this Riemann-Silberstein vector. This will remove the singularity and make all the field lines become linked and revolve around a central circle as described by

$$
\mathbf{F}_{H R}^{\prime}(t, \mathbf{r})=\frac{1}{\left((t-i)^{2}-r^{2}\right)^{3}}\left(\begin{array}{c}
(x-i y)^{2}-(t-i-z)^{2} \\
i(x-i y)^{2}+i(t-i-z)^{2} \\
-2(x-i y)(t-i-z)
\end{array}\right)
$$

This gives precisely the electromagnetic Hopf-Rañada knot. Note that, up to a constant factor, the difference with (172) is the change of the $y \rightarrow z$ and the permutation of the vector components. Fig. 14 illustrates this solution (once more) so that it can be easily compared with the solution before the complex time shift (Fig. 13). Several articles by I. Bialinicky-Birula and Z. BialinickyBirula are closely related to this topic and highly recommended $[63,77,78]$. 

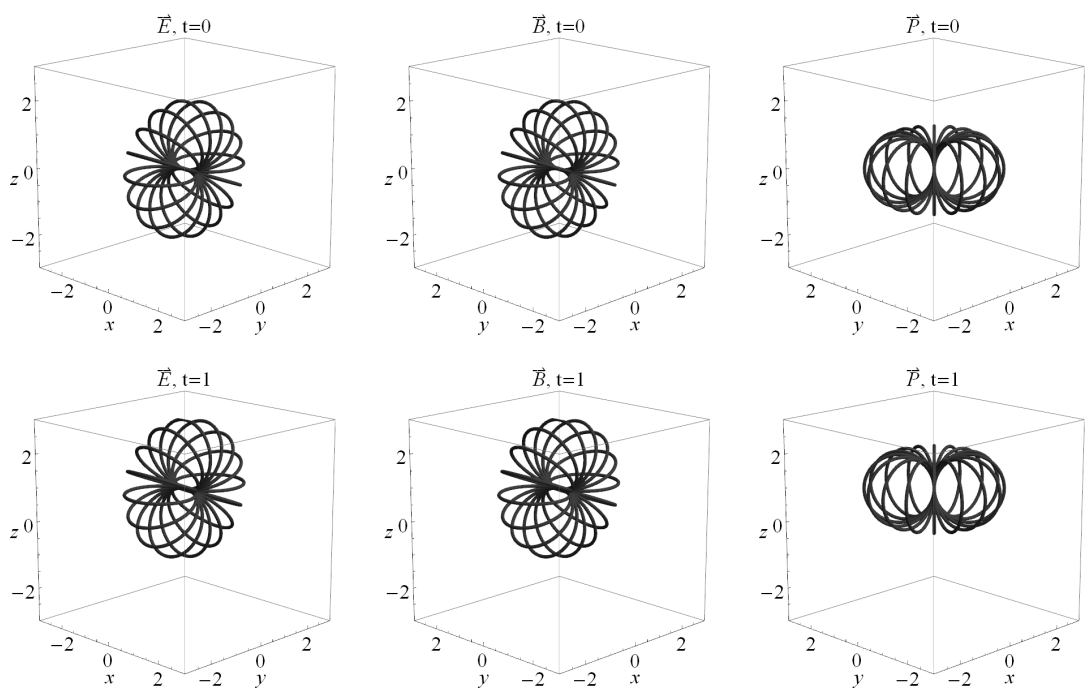

Figure 13: Degenerate field configuration: time evolution of $\mathbf{E}, \mathbf{B}$, and $\mathbf{P}$ field lines at $t=0$ and at $t=1$.
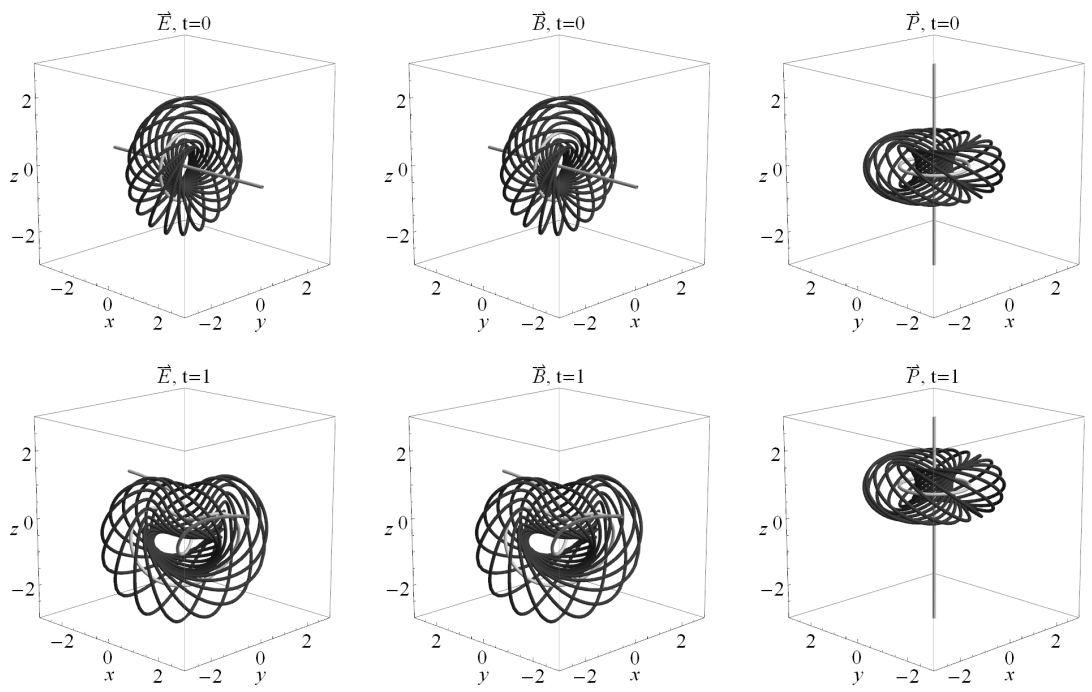

Figure 14: Hopf-Rañada field: time evolution of $\mathbf{E}, \mathbf{B}$, and $\mathbf{P}$ field lines at $t=0$ and at $t=1$. 


\subsection{Generalization of complex shift method}

In [21] the method of generating topologically non-trivial solutions of the source-free Maxwell equations from existing ones by applying conformal transformations with complex parameters is further investigated in terms of Bateman's variables. Bateman's construction presented in Section 6.1 for null electromagnetic fields, in terms of the Riemann-Silberstein vector $\mathbf{F}=\mathbf{E}+i \mathbf{B}$, reads

$$
\mathbf{F}=\nabla \alpha \times \nabla \beta
$$

and (170) is written now as $\nabla \cdot \mathbf{F}=0$, and the dynamical part as $i \partial_{t} \mathbf{F}=\nabla \times \mathbf{F}$. Inserting in this equation the expression (203) yields

$$
i \nabla \times\left(\partial_{t} \alpha \nabla \beta-\partial_{t} \beta \nabla \alpha\right)=\nabla \times(\nabla \alpha \times \nabla \beta),
$$

which is satisfied if

$$
i\left(\partial_{t} \alpha \nabla \beta-\partial_{t} \beta \nabla \alpha\right)=\nabla \alpha \times \nabla \beta,
$$

which is equivalent to some of the conditions (169).

In the previous section it was shown that a constant $\mathbf{E}$ field and an orthogonal B field leads after conformal inversion and a complex time shifts to the HopfRañada field. To reproduce this construction in terms of Bateman's variables we start with the representations for a constant electric and magnetic field in terms of the complex functions $\alpha$ and $\beta$.

$$
\alpha=2 i(t+z)-1, \beta=2(x-i y)
$$

which give $\mathbf{E}=(-4,0,0), \mathbf{B}=(0,4,0)$. Performing a special conformal transformation combined with a complex time shift,

$$
x^{\mu} \rightarrow \frac{x^{\mu}-b^{\mu} x_{\sigma} x^{\sigma}}{1-2 b_{\sigma} x^{\sigma}+b_{\sigma} b^{\sigma} x_{\rho} x^{\rho}}, \text { with } b^{\mu}=(i, 0,0,0),
$$

provides the Hopf-Ranada solution in Bateman's variables,

$$
\alpha=\frac{a-1+i z}{a+i t}, \quad \beta=\frac{(x-i y)}{a+i t}, \quad \text { with } a=\frac{1}{2}\left(x^{2}+y^{2}+z^{2}-t^{2}+1\right) .
$$



equations by considering complex shifts combined with conformal transformations performed on plane waves and Hopfions and characterizes the solutions by their conserved quantities.

\section{The twistor formulation of linked and knotted null fields}

An essential feature of the Hopfion is the fact that the Poynting vector is tangent to a Hopf fibration that propagates at the speed of light without deformation. This Poynting vector structure forms a so called Robinson congruence which plays a central role in twistor theory $[62,14]$.

Twistor theory was developed by Roger Penrose and his colleagues as an attempt to combine general relativity and quantum mechanics in a single formalism $[13,79,80]$. The underlying philosophy of the twistor formalism is that the structure of space time is determined by light rays, or, more general, by massless field configurations, and therefore it might be advantageous to reformulate the laws of physics in coordinates that are directly associated to massless fields. This leads to the concept of twistor space $\mathbb{T}$ in which a point, called a twistor $Z^{\alpha}$, encodes both linear and angular momentum of a massless field. $Z^{\alpha}$ is an object with 4 complex variables $(\alpha=0,1,2,3)$. We provide an introduction to twistor theory and twistor quantization in the Appendix B. There it is shown that the norm in twistor space, for which we also need dual twistors denoted by $\bar{Z}_{\alpha}$, is given by $\mathfrak{h}=\frac{1}{2} Z^{\alpha} \bar{Z}_{\alpha}$. This norm is preserved under conformal transformations (just like in Minkowski space the 4-interval is preserved under Lorentz transformations) and turns out to be the helicity of the massless field configuration associated to the twistor. Canonical quantization of twistor space can be carried out (in exact analogy with introducing non-commuting operators for position and momentum) leading to replacing $Z^{\alpha}$ and $\bar{Z}_{\alpha}$ by operators on twistor function: $\left(\hat{Z}^{\alpha}, \hat{\bar{Z}}_{\beta}\right)=\left(Z^{\alpha},-\frac{\partial}{\partial Z^{\beta}}\right)$. After quantization the twistor norm becomes the helicity operator $\mathfrak{h}=-\frac{1}{2}\left(Z^{\alpha} \frac{\partial}{\partial Z^{\alpha}}+2\right)$ (see Appendix B). It is now a matter of finding the eigenvalues and eigenstates of the helicity operator. 
This gives the so-called elementary states in twistor theory. After transforming the Penrose transform, one obtains all the torus knots in electromagnetism as well as a recipe for similar knots for fields with different spin values.

Next we show that the EM Hopf-Ranada knot is indeed equivalent to a simple nontrivial solution to the spin-1 massless field equation in twistor space.

1015 Then we show that the entire family of torus knots is covered by elementary twistor states. We use the power of the twistor formalism to derive knotted field configurations for other massless fields, in particular for the spin 2 field associated to the linearized Einstein equations in vacuum. We conclude by analyzing the spin-2 solution within the framework of gravito-electromagnetism ${ }_{1020}[81,82]$. By decomposing the spin-2 field into the spatial gravito-electric and gravito-magnetic tensors we characterize their topological structure in terms of the EM knots.

\subsection{EM Hopf-Rañada knot from twistor theory}

Classical electromagnetism [79] resides in the spin-1 sector of the source-free spinor field equations (see (320) in Appendix B):

$$
\begin{array}{r}
\nabla^{A A^{\prime}} \varphi_{A^{\prime} B^{\prime}}=0, \\
F_{a b}=F_{A^{\prime} B^{\prime} A B}=\varphi_{A^{\prime} B^{\prime}} \epsilon_{A B}+\text { c.c. }
\end{array}
$$

Indices $a$ and $b$ have values 1 to 4 , and the spinor indices $A$ and $A^{\prime}$ (and $B$ and $B^{\prime}$ ) have values 0 , and 1 . The standard electric and magnetic fields are recovered by decomposing $F_{a b}$ using a 4 -velocity $u^{a}$ so that

$$
\begin{aligned}
& \mathcal{E}_{b}=u^{a} F_{a b} \\
& \mathcal{B}_{b}=-u^{a *} F_{a b}
\end{aligned}
$$


where $^{*}$ denotes the Hodge dual. Taking $u^{a}=(1,0,0,0)$ we have that

$$
F_{a b}=\left(\begin{array}{cccc}
0 & E_{x} & E_{y} & E_{z} \\
-E_{x} & 0 & -B_{z} & B_{y} \\
-E_{y} & B_{z} & 0 & -B_{x} \\
-E_{z} & -B_{y} & B_{x} & 0
\end{array}\right) .
$$

The corresponding twistor function with helicity value 1 leads after the Penrose transform (see (323) in Appendix B) to the solution,

$$
\varphi_{A_{1}^{\prime} \cdots A_{2 h}^{\prime}}(x)=\left(\frac{2}{\mu_{B} \sigma^{B}|x-y|^{2}}\right)^{3} \mathcal{A}_{A^{\prime}} \mathcal{A}_{B^{\prime}}
$$

where $\mathcal{A}_{A^{\prime}}$ defines the doubly degenerate principle null direction of $F_{a b}$. Choosing

$$
\begin{aligned}
& \bar{A}_{a}=(-i \sqrt{2}, \sqrt{2},-i, 1), \\
& \bar{B}_{\beta}=\frac{\pi^{1 / 3}}{2^{4 / 3}}(-\sqrt{2}, \sqrt{2} i,-1, i)
\end{aligned}
$$

reproduces precisely the Hopf-Rañada field. The expressions for the time dependent electromagnetic fields admit a simple Riemann-Silberstein vector,

$$
\begin{aligned}
\mathbf{F}_{R} & =\mathbf{E}_{R}+i \mathbf{B}_{R} \\
& =\frac{4}{\pi\left(-(t-i)^{2}+r^{2}\right)^{3}}\left(\begin{array}{c}
(x-i z)^{2}-(t-i+y)^{2} \\
2(x-i z)(t-i+y) \\
i(x-i z)^{2}+i(t-i+y)^{2}
\end{array}\right),
\end{aligned}
$$

where $r^{2}=x^{2}+y^{2}+z^{2}$. The energy density and Poynting vector field are

$$
\begin{aligned}
U_{R} & =\frac{16\left(1+x^{2}+(t+y)^{2}+z^{2}\right)^{2}}{\pi^{2}\left(1+2\left(t^{2}+r^{2}\right)+\left(t^{2}-r^{2}\right)^{2}\right)^{3}} . \\
\mathbf{P}_{R} & =\frac{U_{R}}{\left(1+x^{2}+(t+y)^{2}+z^{2}\right)}\left(\begin{array}{c}
2(x(t+y)+z) \\
1+(t+y)^{2}-x^{2}-z^{2} \\
2(z(t+y)-x)
\end{array}\right) .
\end{aligned}
$$

\subsection{Null EM torus knots from twistor theory}

Now we show that the EM field configurations based on the null electromagnetic torus knots are contained within the elementary states of twistor theory. 

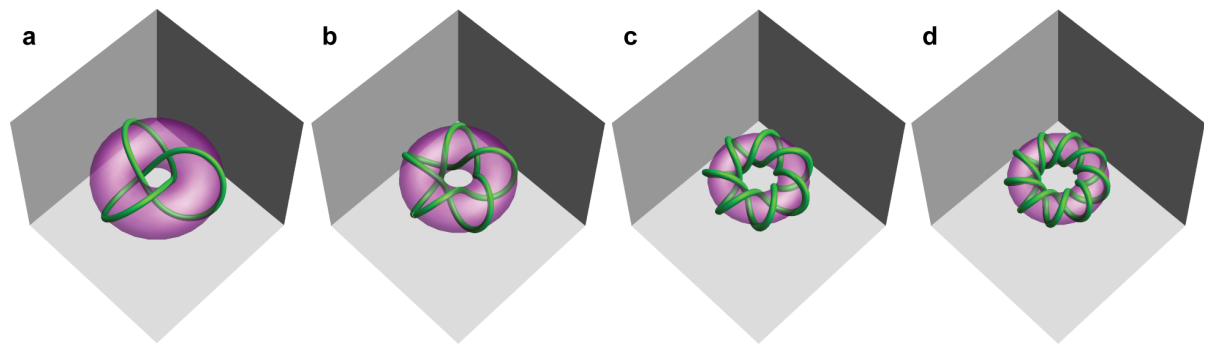

Figure 15: (Colour online) Torus knots (green) wind $\left(n_{t}, n_{p}\right)$ times around a torus (purple) in the toroidal and poloidal directions, respectively. Shown here are the cases of a) trefoil $(2,3)$ knot, b) cinquefoil $(2,5)$ knot, c) septafoil $(2,7)$ knot, and d) nonafoil $(2,9)$ knot.

The Hopf fibration appears as the degenerate case whereby the linked and knotted toroidal structure degenerates down to the linked hopfion configuration.

Torus knots are closed curves on the surface of a torus which wind an integer number of times about the toroidal direction $n_{t}$ and poloidal direction $n_{p}$ as in Fig. 15, where $n_{t}$ and $n_{p}$ are coprime and both greater than one. If $n_{t}$ and $n_{p}$ are not coprime, then there are $n_{g}=\operatorname{gcd}\left(n_{t}, n_{p}\right)$ linked curves, each corresponding to a $\left(n_{t}, n_{p}\right) \bmod n_{g}$ torus knot. If either $n_{t}$ or $n_{p}$ is equal to one, then the knot is trivial with $n_{g}$ linked curves.

Following the twistor program, solutions to the massless spin- $N$ equations in Minkowski space $M$ are obtained via the Penrose transform of functions $f(Z)$ in twistor space [80]. Consider the twistor functions corresponding to the elementary states [13]

$$
f(Z)=\frac{\left(\bar{C}_{\gamma} Z^{\gamma}\right)^{c}\left(\bar{D}_{\delta} Z^{\delta}\right)^{d}}{\left(\bar{A}_{\alpha} Z^{\alpha}\right)^{a}\left(\bar{B}_{\beta} Z^{\beta}\right)^{b}}
$$

where $\left(\bar{A}_{\alpha} Z^{\alpha}\right)$ is the $S U(2,2)$ twistor inner product. Choosing $a=1$ yields null/Type $\mathrm{N}$ solutions and we must have $b=2 h+1+c+d$ to give the correct homogeneity $n=-2 h-2$ for a solution with helicity $h$. In this section it is shown that the twistor functions of the form

$$
f(Z)=\frac{\left(\bar{C}_{\gamma} Z^{\gamma}\right)^{h\left(n_{p}-1\right)}\left(\bar{D}_{\delta} Z^{\delta}\right)^{h\left(n_{t}-1\right)}}{\left(\bar{A}_{\alpha} Z^{\alpha}\right)\left(\bar{B}_{\beta} Z^{\beta}\right)^{h\left(n_{p}+n_{t}\right)+1}},
$$

lead to field configurations with a torus knot topology where $n_{p}$ and $n_{t}$ correspond to the poloidal and toroidal winding numbers. 
After applying the spin-1 Penrose transform to (218), the resulting spinor field is

$$
\psi_{A^{\prime} B^{\prime}}(x)=\frac{\left(\mathcal{A}_{C^{\prime}} \mathcal{C}^{C^{\prime}}\right)^{n_{p}-1}\left(\mathcal{A}_{D^{\prime}} \mathcal{D}^{D^{\prime}}\right)^{n_{t}-1}}{\left(\mathcal{A}_{E^{\prime}} \mathcal{B}^{E^{\prime}}\right)^{n_{p}+n_{t}+1}} \mathcal{A}_{A^{\prime}} \mathcal{A}_{B^{\prime}}
$$

Note that the Latin script spinor variables are the spinors associated to the Latin twistor variable. Ergo, $\mathcal{A}_{A^{\prime}}$ is defined implicitly by $\bar{A}_{\alpha} Z^{\alpha}=\mathcal{A}_{A^{\prime}} \pi^{A^{\prime}}$.

For the following choice of the dual twistors

$$
\begin{aligned}
\bar{A}_{\alpha} & =i(0, \sqrt{2}, 0,1) \\
\bar{B}_{\beta} & =i(-\sqrt{2}, 0,-1,0) \\
\bar{C}_{\gamma} & =(0,-\sqrt{2}, 0,1) \\
\bar{D}_{\delta} & =i(-\sqrt{2}, 0,1,0),
\end{aligned}
$$

the spin-1 fields are null torus knots with a Poynting vector that is everywhere tangent to a Hopf fibration and propagates in the $\hat{z}$-direction without deformation. The solutions have the same topology as the electromagnetic fields in [19]. The electric and magnetic vector fields each have the following topological structure as shown in Fig. 16. There are $2 n_{g}$ core field lines, where $n_{g}=\operatorname{gcd}\left(n_{t}, n_{p}\right)$, which are linked (and knotted if $n_{t}, n_{p}>1$ ). Each core line has the same configuration as the corresponding torus knot with $\left(n_{t}, n_{p}\right)$ shown in Fig. 15. With the choice for $C$ and $D$ given in (220), the poloidal and toroidal winding numbers for the EM case are related to the exponents in (217) by $c=n_{p}-1$ and $d=n_{t}-1$. A single core field line is surrounded by nested, toroidal surfaces, each filled by one field line. A second core field line, also surrounded by nested surfaces, is linked with the first so that there are $2 n_{g}$ sets of linked nested surfaces which fill all of space. A complete solution to the Maxwell equations consists of an electric and a magnetic field orthogonal to each other, both with this field line structure. The $(1,1)$ case corresponds to the electromagnetic Hopfion.

At $t=0$ the electric and magnetic fields are tangent to orthogonal torus knots, as shown in Fig. 17 (first row). The fields will deform under time evolution, but the topology will be conserved since $\mathbf{E} \cdot \mathbf{B}=0$. 


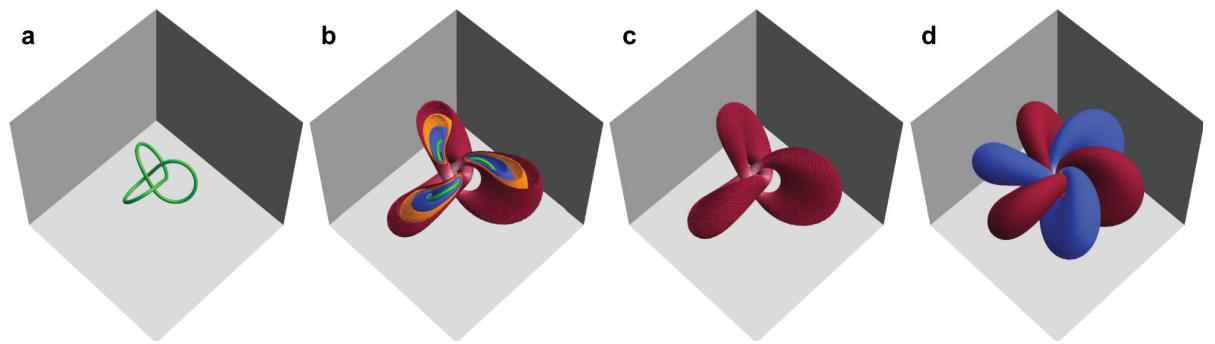

Figure 16: (Colour online) The field line structure based on $(2,3)$ trefoil knot. a) The core field line is a torus knot (green). b) Each field line except the core lies on the surface of a nested, deformed torus. c) One field line fills a complete surface (red). d) Another field line fills a second surface (blue) linked with the first. The two linked core field lines and the nested surfaces around them fill all of space.

\subsection{Gravito-electromagnetic Hopf Knot}

Linearized general relativity makes up the spin- 2 sector of the spin- $N$ equations [79]. The field equation and associated field strength spinor are given by

$$
\begin{aligned}
\nabla^{A A^{\prime}} \varphi_{A^{\prime} B^{\prime} C^{\prime} D^{\prime}} & =0, \\
C_{A^{\prime} B^{\prime} C^{\prime} D^{\prime} A B C D} & =\varphi_{A^{\prime} B^{\prime} C^{\prime} D^{\prime}} \epsilon_{A B} \epsilon_{C D}+\text { c.c. }
\end{aligned}
$$

In analogy with the spin-1 case, following [81] and [82], we decompose the Weyl tensor using a 4-velocity $u^{a}$ into a tidal (gravito-electric) field and frame-drag (gravito-magnetic) field,

$$
\begin{aligned}
& \mathcal{E}_{a c}=u^{b} u^{d} C_{a b c d} \\
& \mathcal{B}_{a c}=-u^{b} u^{d *} C_{a b c d} .
\end{aligned}
$$

Taking $u^{a}=(1,0,0,0)$ we define the spatial tensors $E_{i j}$ and $B_{i j}$

$$
\begin{aligned}
& E_{i j}=C_{i 0 j 0} \\
& B_{i j}=-{ }^{*} C_{i 0 j 0} .
\end{aligned}
$$

The symmetric traceless tensors $E_{i j}$ and $B_{i j}$ are called tidal and frame-drag fields since two orthogonal observers separated by a small spatial vector $\boldsymbol{\xi}$ will 

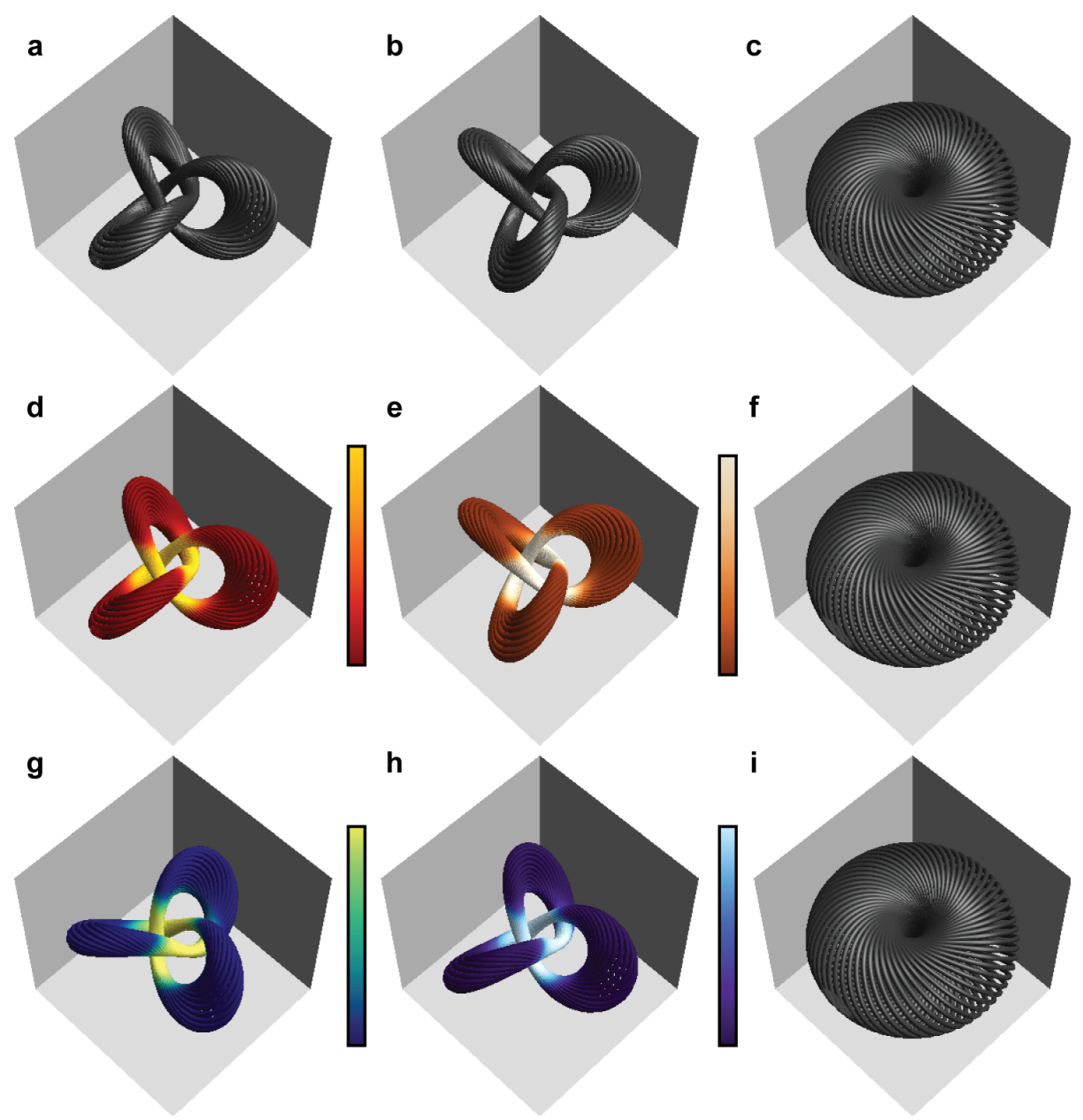

Figure 17: (Colour online) A comparison of the spin-1 (EM) and spin-2 (gravity) trefoil knots at $t=0$. The first row is the EM trefoil knot: $\mathbf{a}$ the electric field, $\mathbf{b}$ the magnetic field, and $\mathbf{c}$ the Poynting vector field. The second row is the gravito-electric trefoil knot: $\mathbf{d}$ the negative eigenvalue field $\vec{e}_{-}$, e the positive eigenvalue field $\vec{e}_{+}$, and $\mathbf{f}$ the zero eigenvalue field $\vec{e}_{0}$. The third row is the gravito-magnetic trefoil knot: $\mathrm{g}$ the negative eigenvalue field $\vec{b}_{-}, \mathbf{h}$ the positive eigenvalue field $\vec{b}_{+}$, and $\mathbf{i}$ the zero eigenvalue field $\vec{b}_{0}$. The color scale indicates magnitude of the eigenvalue, with lighter colors indicating a higher magnitude. 
experience a relative tidal acceleration given by

$$
\Delta a_{i}=-E_{i j} \xi^{j}
$$

and a gyroscope at the tip of $\boldsymbol{\xi}$ will precess with angular velocity

$$
\Delta \Omega_{i}=B_{i j} \xi^{j}
$$

relative to inertial frames at the tail of $\boldsymbol{\xi}$. Since the tidal and frame-drag fields are symmetric and traceless, each may be characterized entirely by its eigenspaces. Thus, if $\boldsymbol{v}$ is an eigenvector of $E$ or $B$ then the integral curves of $\boldsymbol{v}$ are the gravitational analog of field lines. The tidal field stretches or compresses objects, and its associated field lines are referred to as tendex lines. The frame-drag field rotates gyroscopes, and its associated field lines are referred to as vortex lines. The tidal field has an associated eigenvalue $E_{\boldsymbol{v}}$ which has a physical interpretation given by the tidal acceleration equation (224). Thus, if the tendex eigenvalue is negative (respectively, positive) then an object oriented along the tendex line is stretched (compressed) along the tendex line. Similar relations hold for the frame-drag field whose eigenvalues are interpreted using 1070 (225), where an object oriented along a vortex line observes counter-clockwise (clockwise) precession of gyroscopes around the vortex line.

The gravito-electromagnetic knot (GEM knot) is constructed in the same fashion as the electromagnetic knot since linearized gravitational fields are taken to be spin-2 fields on $M$. Thus, taking $h=2$ in (323), we have

$$
\varphi_{A_{1}^{\prime} \cdots A_{2 h}^{\prime}}(x)=\left(\frac{2}{\Omega|x-y|^{2}}\right)^{5} \mathcal{A}_{A^{\prime}} \mathcal{A}_{B^{\prime}} \mathcal{A}_{C^{\prime}} \mathcal{A}_{D^{\prime}}
$$

where $\mathcal{A}_{A^{\prime}}$ defines the totally degenerate principal null directions of $C_{a b c d}$ making it a Petrov type $\mathrm{N}$ linearized gravitational field. Taking the dual twistors $\bar{A}_{\alpha}$ and $\bar{B}_{\beta}$ to be the same as in the electromagnetic knot we construct the Weyl curvature from (221). Then we perform the gravito-electric and gravito-magnetic decompositions as in (223).

The tidal and frame-drag fields are characterized by their spectral decomposition which provides a three dimensional picture of the space-time via the 
a

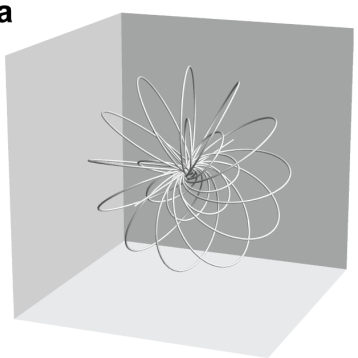

d

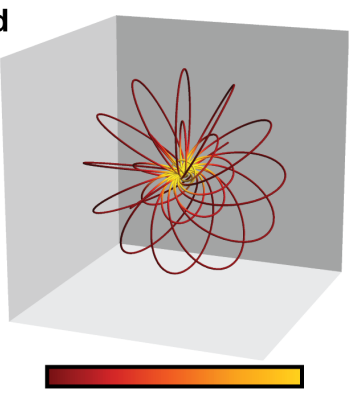

g

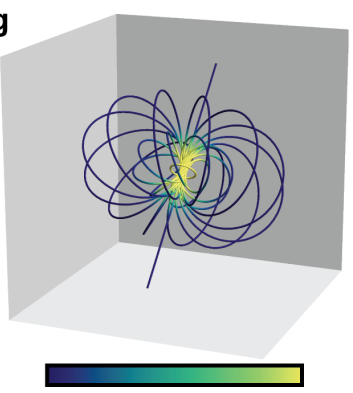

b

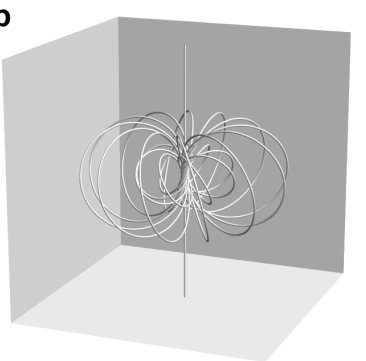

e

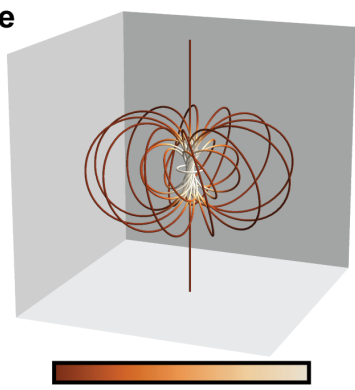

h

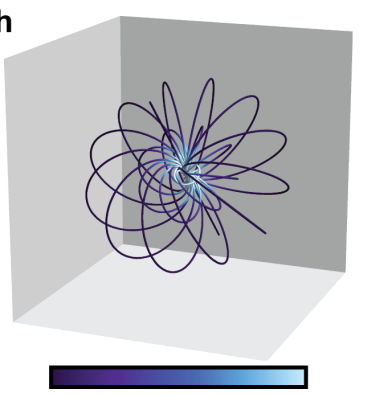

C

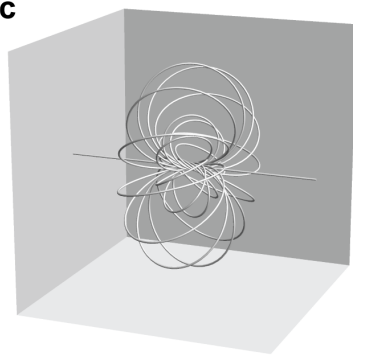

f

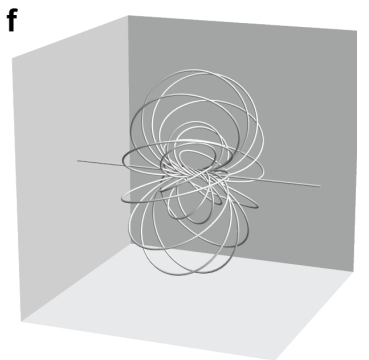

i

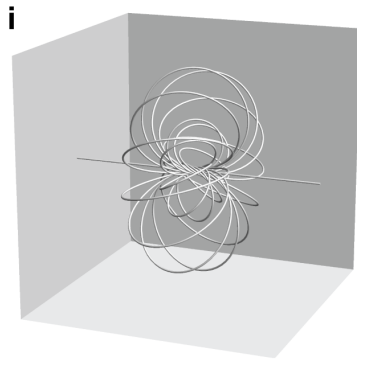

Figure 18: (Colour online) A comparison of the spin-1 (EM) and spin-2 (gravity) knots at $t=0$. In the first row, we have the EM knot: $\mathbf{a}$ the electric field, $\mathbf{b}$ the magnetic field, and $\mathbf{c}$ the Poynting vector field. In the second row, we have the gravito-electric knot: $\mathbf{d}$ the negative eigenvalue field $E_{-}$, e the positive eigenvalue field $E_{+}$, and $\mathbf{f}$ the zero eigenvalue field $E_{0}$. In the third row, we have the gravito-magnetic knot: $\mathbf{g}$ the negative eigenvalue field $B_{-}, \mathbf{h}$ the positive eigenvalue field $B_{+}$, and $\mathbf{i}$ the zero eigenvalue field $B_{0}$. The color scale indicates magnitude of the eigenvalue, with lighter colors indicating a higher magnitude. 
integral curves of their eigenvector fields and their various physical interpretations. Performing the spectral decomposition we find that both fields possess an eigenvalue structure $\{+\Lambda,-\Lambda, 0\}$ corresponding respectively to the eigenvectors $\left\{\mathbf{E}_{+}, \mathbf{E}_{-}, \mathbf{E}_{0}\right\}$ (and $\left\{\mathbf{B}_{+}, \mathbf{B}_{-}, \mathbf{B}_{0}\right\}$ ) where

$$
\Lambda(x)=\frac{2^{8 / 3}\left(1+x^{2}+(y+t)^{2}+z^{2}\right)^{2}}{\pi^{5 / 3}\left(1+2\left(t^{2}+r^{2}\right)+\left(t^{2}-r^{2}\right)^{2}\right)^{5 / 2}}
$$

is the positive eigenvalue which determines the strength of the interaction, for both the tidal and frame-drag fields.

Considering first the eigenvector fields which correspond to the zero eigenvalue, we find that

$$
\begin{aligned}
\mathbf{E}_{0} & =\mathbf{B}_{0}=\left(\begin{array}{c}
2(x(t+y)+z) \\
1+(t+y)^{2}-x^{2}-z^{2} \\
2(z(t+y)-x)
\end{array}\right) \\
& =\left(1+x^{2}+(t+y)^{2}+z^{2}\right) \frac{\mathbf{P}_{R}}{\left|\mathbf{P}_{R}\right|}
\end{aligned}
$$

which is, up to an overall scaling function, the Poynting vector of the EM knot. Constructing the Riemann-Silberstein structures for the remaining fields $\left\{\boldsymbol{E}_{-}, \boldsymbol{E}_{+}\right\}$and $\left\{\boldsymbol{B}_{-}, \boldsymbol{B}_{+}\right\}$

$$
\begin{aligned}
& \mathbf{F}_{G E}=\mathbf{E}_{-}+i \mathbf{E}_{+}, \\
& \mathbf{F}_{G B}=\mathbf{B}_{-}+i \mathbf{B}_{+},
\end{aligned}
$$

we find that

$$
\begin{aligned}
\mathbf{F}_{G E} & =e^{i \pi / 4} \mathbf{F}_{G B} \\
& =e^{i \operatorname{Arg}(\vartheta)} \mathbf{F}_{R},
\end{aligned}
$$

where

$$
\vartheta=\sqrt{-(t-i)^{2}+r^{2}} .
$$

This defines the tidal and frame-drag fields in terms of the EM knot. Equation (231) shows that the frame-drag field is a rotation of the tidal field about the 
a

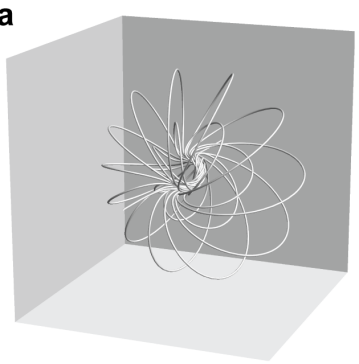

d

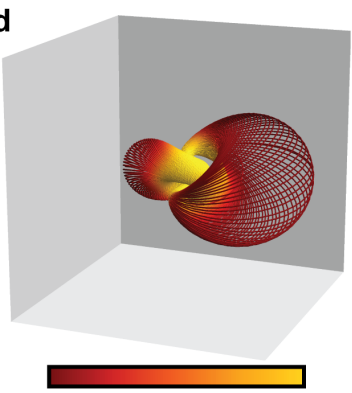

g

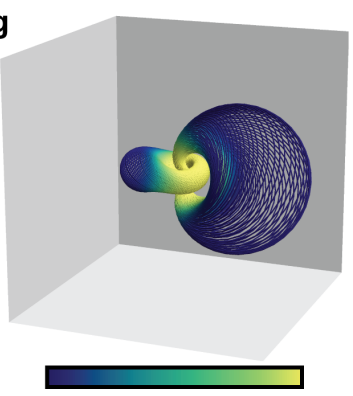

b

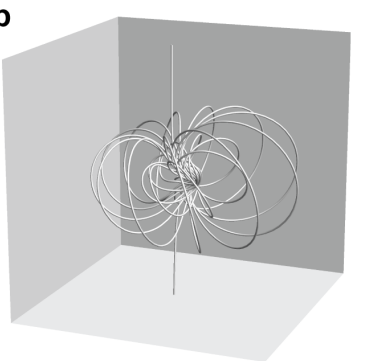

e

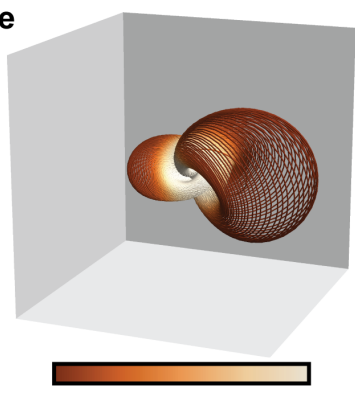

h

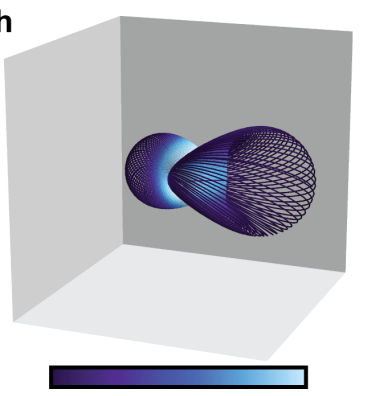

C

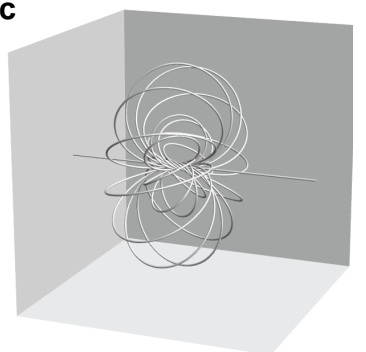

f

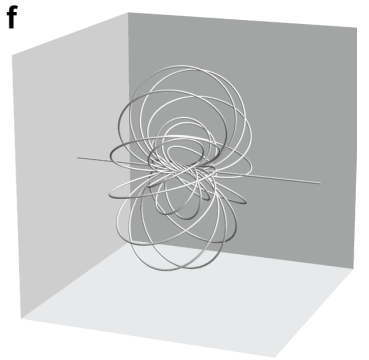

i

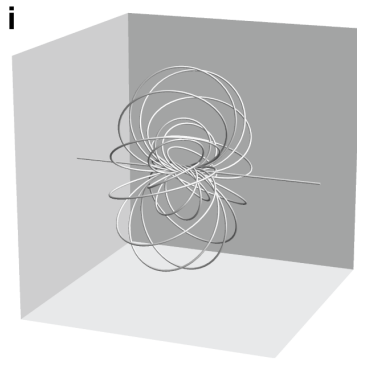

Figure 19: (Colour online) A comparison of the spin-1 (EM) and spin-2 (gravity) knots at $t=1$. In the first row, we have the EM knot: $\mathbf{a}$ the electric field, $\mathbf{b}$ the magnetic field, and $\mathbf{c}$ the Poynting vector field. In the second row, we have the gravito-electric knot: $\mathbf{d}$ the negative eigenvalue field $E_{-}$, e the positive eigenvalue field $E_{+}$, and $\mathbf{f}$ the zero eigenvalue field $E_{0}$. In the third row, we have the gravito-magnetic knot: $\mathbf{g}$ the negative eigenvalue field $B_{-}, \mathbf{h}$ the positive eigenvalue field $B_{+}$, and $\mathbf{i}$ the zero eigenvalue field $B_{0}$. The color scale indicates magnitude of the eigenvalue, with lighter colors indicating a higher magnitude. 
Poynting vector of the EM knot. Thus in passing to spin-2 we obtain two independent gravito-knot structures: a gravito-electric knot and a gravito-magnetic knot which differ simply by a global rotation. Equation (232) shows that the tidal field is simply a local duality transformation of the EM knot. At $t=0$, $\operatorname{Arg}(\vartheta)=0$ and thus provides no duality transformation. Hence the tendex structure of the tidal field is the same as the field line structure of the EM knot. Furthermore, the vortex lines are the same as the tendex lines but rotated by $45^{\circ}$ in the $x y$-plane. Thus the $t=0$ tendex and vortex configuration is given by the six Hopf fibrations of Fig. 18 (rows 2 and 3) so that for each fixed eigenvalue the associated tendex (vortex) lines are closed and linked exactly once. For $t \neq 0, \vartheta$ is complex and hence the tendex lines differ from those of the EM knot by a local duality transformation as shown in Fig. 19 with the vortex structure different again by only a rotation of $45^{\circ}$.

For electromagnetic fields, there are two quantities that are invariant under a local duality rotation - the energy density and Poynting vector. For the EM knot, these are given in (215) and (216). In direct analogy with the local duality invariants of electromagnetism, a covariant super-energy density and super-Poynting vector that are invariant under a local duality rotation can be defined [81]. For the GEM knot, we find the super-energy density and superPoynting vector are given by

$$
\begin{aligned}
U_{G} & =\frac{1}{2}\left(E_{a b} E^{a b}+B_{a b} B^{a b}\right) \\
& =\frac{64 \cdot 2^{1 / 3}\left(1+x^{2}+(t+y)^{2}+z^{2}\right)^{4}}{\pi^{10 / 3}\left(1+2\left(t^{2}+r^{2}\right)+\left(t^{2}-r^{2}\right)^{2}\right)^{5}} \\
\left(P_{G}\right)_{a} & =\epsilon_{a b c} E_{d}^{b} B^{c d} \\
& =\frac{U_{G}}{\left(1+x^{2}+(t+y)^{2}+z^{2}\right)}\left(\begin{array}{c}
2(x(t+y)+z) \\
1+(t+y)^{2}-x^{2}-z^{2} \\
2(z(t+y)-x)
\end{array}\right) .
\end{aligned}
$$

There is a striking similarity between the local duality invariants for the spin-1 and spin-2 cases, which differ only by the power of the scalar factor which shows the energy falls off more rapidly for the higher spin fields. 


\subsection{Gravito-electromagnetic torus knots}

Following [83] we applying the spin-2 Penrose transform to (218), the resulting spinor field is

$$
\phi_{A^{\prime} B^{\prime} C^{\prime} D^{\prime}}(x)=\frac{\left(\mathcal{A}_{F^{\prime}} \mathcal{C}^{F^{\prime}}\right)^{2\left(n_{p}-1\right)}\left(\mathcal{A}_{G^{\prime}} \mathcal{D}^{G^{\prime}}\right)^{2\left(n_{t}-1\right)}}{\left(\mathcal{A}_{E^{\prime}} \mathcal{B}^{E^{\prime}}\right)^{2\left(n_{p}+n_{t}\right)+1}} \mathcal{A}_{A^{\prime}} \mathcal{A}_{B^{\prime}} \mathcal{A}_{C^{\prime}} \mathcal{A}_{D^{\prime}}
$$

The source-free field equation and Weyl field strength spinor are

$$
\begin{aligned}
\nabla^{A A^{\prime}} \varphi_{A^{\prime} B^{\prime} C^{\prime} D^{\prime}} & =0, \\
C_{A^{\prime} B^{\prime} C^{\prime} D^{\prime} A B C D} & =\varphi_{A^{\prime} B^{\prime} C^{\prime} D^{\prime}} \epsilon_{A B} \epsilon_{C D}+\text { c.c. }
\end{aligned}
$$

The Weyl tensor can then be decomposed into the GEM components. For Type $\mathrm{N}$, the eigenvalues for both the GE and GM tensors take the form $\{-\Lambda, 0,+\Lambda\}$, with $-\Lambda(x) \leq 0 \leq+\Lambda(x)$ for all points $x$ in space-time The magnitude of the eigenvalues are

$\Lambda=\frac{2^{2 n_{p}-3}\left(1+r^{2}+t^{2}-2 t z\right)^{2}\left(r^{2}-z^{2}\right)^{n_{p}-1}\left(r^{4}-2 r^{2}\left(1+t^{2}\right)+\left(1+t^{2}\right)^{2}+4 z^{2}\right)^{n_{t}-1}}{\left(r^{4}-2 r^{2}\left(-1+t^{2}\right)+\left(1+t^{2}\right)^{2}\right)^{\frac{5}{2}+n_{t}+n_{p}}}$

We label the eigenvectors $\left\{\vec{e}_{-}, \vec{e}_{0}, \vec{e}_{+}\right\}$and $\left\{\vec{b}_{-}, \vec{b}_{0}, \vec{b}_{+}\right\}$corresponding to the eigenvalues for the tidal and frame-drag fields respectively. For the zero eigenvalue, the eigenvectors $\vec{e}_{0}$ and $\vec{b}_{0}$ are both aligned with the Poynting vector of the null EM torus knots. For the remaining eigenvectors, we can construct Riemann-Silberstein (RS) vectors $\vec{f}_{e}=\vec{e}_{-}+i \vec{e}_{+}$and $\vec{f}_{b}=\vec{b}_{-}+i \vec{b}_{+}$which are related to each other by

$$
\overrightarrow{f_{e}}=e^{i \pi / 4} \vec{f}_{b}
$$

At $t=0$, the eigenvectors of the GE fields have precisely the same structure as the EM fields, and the GM eigenvector fields have the same structure but rotated by $45^{\circ}$. The surfaces of the $\vec{e}_{-}$eigenvector, color-scaled according to the magnitude of the eigenvalue, for different values of $\left(n_{t}, n_{p}\right)$ are shown in Fig. 20. The other GEM fields can be constructed by rotating $\vec{e}_{-}$according to (240): $\vec{e}_{+}$is found by rotating $\vec{e}_{-}$by $90^{\circ}$ about the Poynting vector. $\vec{b}_{-}$and $\vec{b}_{+}$ are found by rotating $\vec{e}_{-}$and $\vec{e}_{+}$by $45^{\circ}$, respectively. The eigenvalues of the GEM fields for a given $\left(n_{t}, n_{p}\right)$ have the same magnitude (color-scaling) given by $|\Lambda(x)|$ in (239). 


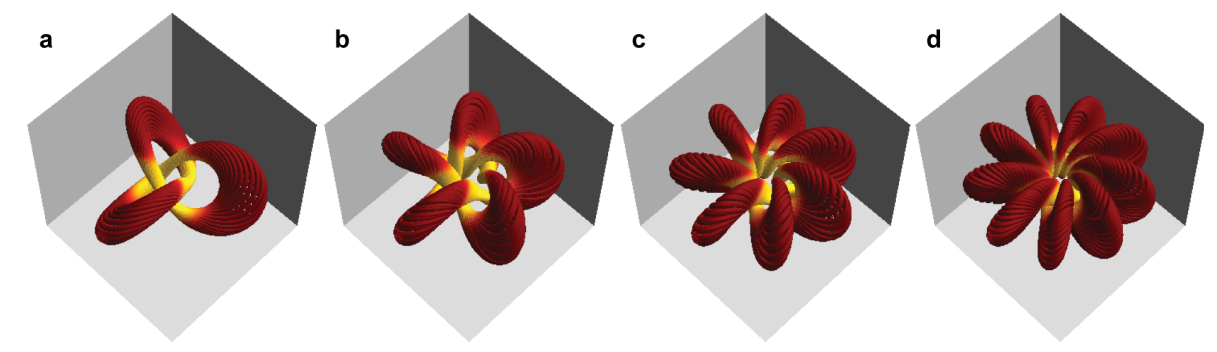

Figure 20: (Colour online) The eigenvector field $\vec{e}_{-}$for the gravitational field based on the a) trefoil $(2,3)$ knot, b) cinquefoil $(2,5)$ knot, c) septafoil $(2,7)$ knot, and d) nonafoil $(2,9)$ knot. The color scaling is the same as in Fig. 17.

\section{Plasma with a magnetic knot skeleton}

The topologically non-trivial EM fields can be considered in the context of plasma physics were Maxwell's equations are combined with fluid dynamics, giving rise to magnetohydrodynamics (MHD). Kamchatnov [11] has explored the Hopf map in a similar way as in the case of electromagnetism but he restricted his attention to a static magnetic field configuration which forms the skeleton of a plasma configuration. The linked magnetic field structure defines a stationary plasma current and pressure configuration in the case of ideal (non-resistive)

1115 MHD. He showed that the conservation of energy, angular momentum and the topological quantity of magnetic helicity give stability to the configuration. Such a configuration is referred to as a topological soliton which is a meta stable state. It is not in an equilibrium, or lowest energy, state, but is shielded from decay by a conserved topological quantity. The energy and angular momentum are functions of scale factors, typically the size $R$ of the soliton, and the magnetic field strength $B_{0}$ at the centre of the soliton. Combined with a third conserved quantity, here the magnetic helicity, the values of $R$ and $B_{0}$ become uniquely defined so that the energy and angular momentum become pinned at a fixed value.

In this section the topological soliton of Kamchatnov is presented and generalized to a class of topological plasma configurations characterized by their 
toroidal and poloidal winding numbers, $n_{t}$ and $n_{p}$ respectively [31]. It will be shown that for positive integers $n_{t}$ and $n_{p}=1$ these configurations represent stable, localized solutions to the MHD equations for an ideal incompressible fluid with infinite conductivity. The section concludes by considering plasma with finite conductivity and by estimating the soliton lifetime in such a medium as a function of the toroidal winding number.

\subsection{Plasma knots}

Because of the importance of topology in plasma dynamics, there has previously been interest in generalizing the Kamchatnov soliton [84]. The topology of field lines has been shown to be related to stability of flux tube configurations, with the helicity placing constraints on the relaxation of magnetic fields in plasma $[85,86]$. Magnetic helicity gives a measure of the structure of a magnetic field, including properties such as twisting, kinking, knotting, and linking $[87,88]$. Simulations have shown that magnetic flux tubes with linking possess a longer decay time than similar configurations with zero linking number [89, 90, 91]. Higher order topological invariants have also been shown to place additional constraints on the evolution of the system [85, 92, 93].

There are many applications where magnetic field topology has a significant effect on the stability and dynamics of plasma systems. For example, toroidal magnetic fields increase confinement in fusion reactors [94, 95], and solving for the behavior of some magnetic confinement systems is only tractable in a coordinate system based on a known parameterization of the nested magnetic surface topology [96, 97, 95]. In Astrophysics, the ratio of the toroidal and poloidal winding of the internal magnetic fields impacts many properties of stars, including the shape [98, 99] and momentum of inertia [100], as well as the gravity wave signatures [101] and disk accretion [102] of neutron stars. The novel class of quasi stable, analytic MHD solutions presented in this section may be of use in the study of fusion reactions, stellar magnetic fields, and plasma dynamics in general.

Within ideal MHD a plasma with a pressure field $p(\mathbf{x})$ and current $\mathbf{j}(\mathbf{x})$ in 

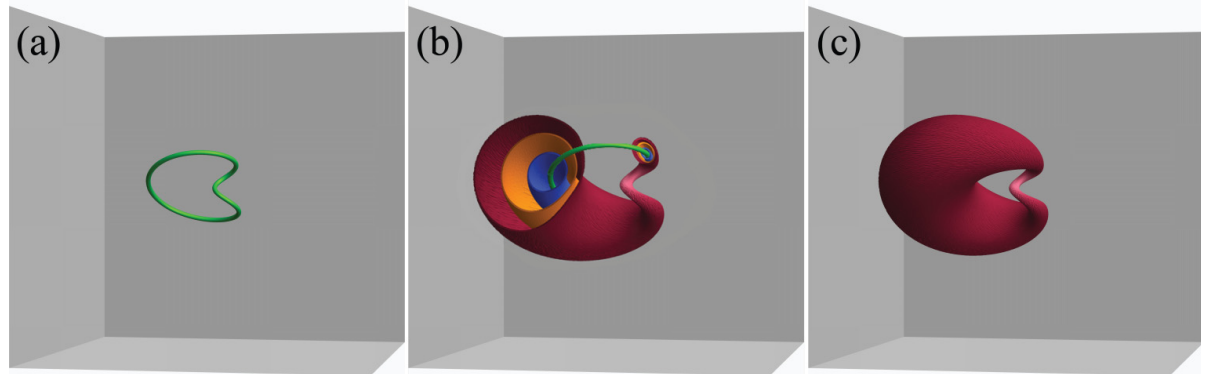

Figure 21: One lobe of the field configuration for $n_{p}=1$ and $n_{t}=2$. (a) A single, closed core magnetic field line. (b) The core field line is surrounded by nested toroidal surfaces, shown in cross section. (c) A complete magnetic surface filled entirely by one field line.

static equilibrium can be described by

$$
\begin{aligned}
\nabla p & =\mathbf{j} \times \mathbf{B}, \\
\nabla \times \mathbf{B} & =\mathbf{j}, \\
\nabla \cdot \mathbf{B} & =0 .
\end{aligned}
$$

Each magnetic surface corresponds to a constant pressure surface, $\mathbf{B} \cdot \nabla p=$ 0 . The time independent magnetic field of the Kamchatnov MHD topological soliton is identical to the magnetic field of the Hopf-Rañada solution at $t=0$.

$$
\mathbf{B}_{\text {soliton }}(\mathbf{r})=\mathbf{B}_{\text {hopfion }}(t=0, \mathbf{r}) .
$$

The soliton field is then sourced by a stationary current

$$
\mathbf{j}(\mathbf{x})=\frac{1}{\mu_{0}} \nabla \times \mathbf{B}_{\text {soliton }}(\mathbf{r}) .
$$

Generalized topological soliton fields can be constructed using (241) and (242) applied to the null radiative torus knots. In other words the timeindependent magnetic field of the soliton is identical to the magnetic field of the radiative torus knots at $t=0$.

The torus knots are constructed from the Bateman potentials (176),

$$
\begin{aligned}
\alpha & =\frac{\left(r^{2}-t^{2}-1\right)+2 i z}{r^{2}-(t-i)^{2}} \\
\beta & =\frac{2(x-\imath y)}{r^{2}-(t-i)^{2}} .
\end{aligned}
$$



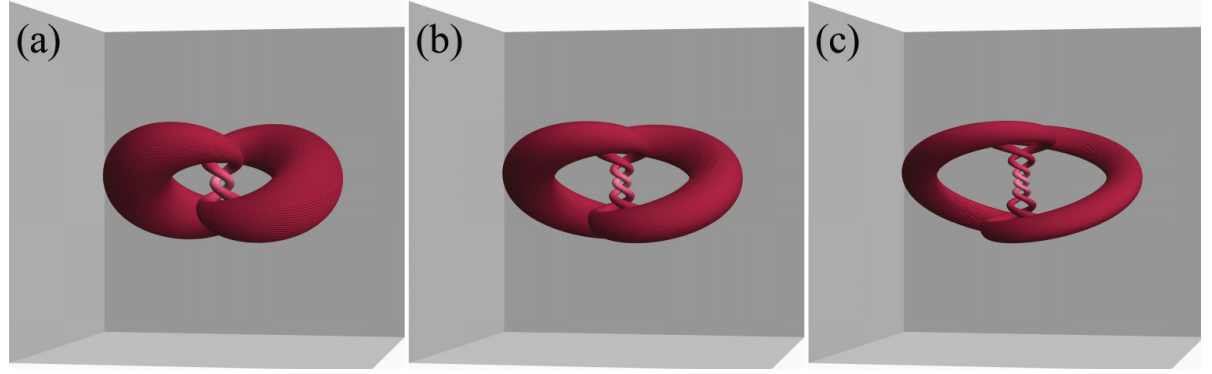

Figure 22: Topological solitons in MHD with $n_{p}=1$ and (a) $n_{t}=2$, (b) $n_{t}=3$, and (c) $n_{t}=4$. A single magnetic field line fills out each of the linked, toroidal surfaces.

The magnetic field of the torus knots is obtained from the expression

$$
\mathbf{B}=\left.\Im\left[\nabla \alpha^{n_{t}} \times \nabla \beta^{n_{p}}\right]\right|_{t=0}
$$

Each $\left(n_{t}, n_{p}\right)$ with $n_{t}, n_{p}=1,2,3 \ldots$ represents a solution to the Maxwell equations. A single magnetic field line fills the entire surface of a torus. These tori are nested and each degenerates down to a closed core field line that winds $n_{t}$ times around the toroidal direction and $n_{p}$ times around the poloidal direction, as illustrated in Fig. 21. A complete solution for a given $\left(n_{t}, n_{p}\right)$ is composed of pairs of these nested surfaces that are linked and fill all of space as shown in Fig. 22. For $n_{g}=\operatorname{gcd}\left(n_{t}, n_{p}\right)$, the solution is a magnetic field with $2 n_{g}$ linked core field lines (knotted if $n_{t}>1$ and $n_{p}>1$ ). If $n_{t}=1$ and $n_{p}=1$, the solution is the Kamchatnov soliton.

\subsection{Stability Analysis}

Following [31] we will study the stability of those knotted structures. First the plasma is considered to be an ideal, perfectly conducting, incompressible fluid, and subsequently the effect of finite conductivity will be considered.

Consider the case where the poloidal winding number $n_{p}=1$ and the toroidal winding number $n_{t}$ is any positive integer. To analyze the stability of the plasma configurations, following the stability analysis in [11], consider the two scaling parameters of the system - the length scale $R$ which corresponds to the size of 
the soliton and $B_{0}$ which is the magnetic field strength at the origin. The length scale $R$ is also the radius of the sphere $S^{3}$ before stereographic projection. First change to coordinates given by:

$$
\{x, y, z\} \rightarrow\left\{\frac{x}{R}, \frac{y}{R}, \frac{z}{R}\right\}, \quad \text { and } \quad|\mathbf{B}(0,0,0)|=B_{0} .
$$

The stability depends on three quantities - energy, magnetic helicity, and angular momentum - which are functions of $R$ and $B_{0}$. For a perfectly conducting plasma, the magnetic helicity $h_{m}$ is an integral of motion and is thus conserved. The magnetic helicity is also a topological invariant proportional to the linking number of the magnetic field lines. If the field can evolve into a lower energy state by a continuous deformation (therefore preserving the topological invariant) then it will be unstable. However, it will be shown that such a deformation does not exist because the angular momentum $\mathbf{L}$ is also conserved and serves to inhibit the spreading of the soliton.

Except for a constant factor, here the magnetic helicity is defined as (53),

$$
h_{m}=\int \mathbf{A} \cdot \mathbf{B} d^{3} r
$$

where $\mathbf{A}=\Im\left[\alpha^{n_{t}} \nabla \beta^{n_{p}}\right]$ is the vector potential. From (243)-(245), it follows that

$$
h_{m}=\frac{2 n_{t}}{\left(n_{t}+1\right)} \pi^{2} B_{0}^{2} R^{4} .
$$

The MHD equations for stationary flow are satisfied for a fluid with velocity

$$
\mathbf{v}= \pm \frac{\mathbf{B}}{\left(\mu_{0} \rho\right)^{\frac{1}{2}}} .
$$

The energy of the soliton is given by

$$
\mathcal{E}=\int\left(\frac{\rho v^{2}}{2}+\frac{B^{2}}{2 \mu_{0}}\right) d^{3} r=\int \frac{3 B^{2}}{2 \mu_{0}} d^{3} r,
$$

so that

$$
\mathcal{E}=\frac{3 n_{t} \pi^{2}}{\mu_{0}} B_{0}^{2} R^{3} \propto \frac{h_{m}}{R} .
$$

The angular momentum is

$$
\mathbf{L}=\rho \int[\mathbf{r} \times \mathbf{v}] d^{3} r=\left(\frac{\rho}{\mu_{0}}\right)^{1 / 2} 4 n_{t} \pi^{2} B_{0} R^{4} h_{m} \mathbf{u}_{y}
$$




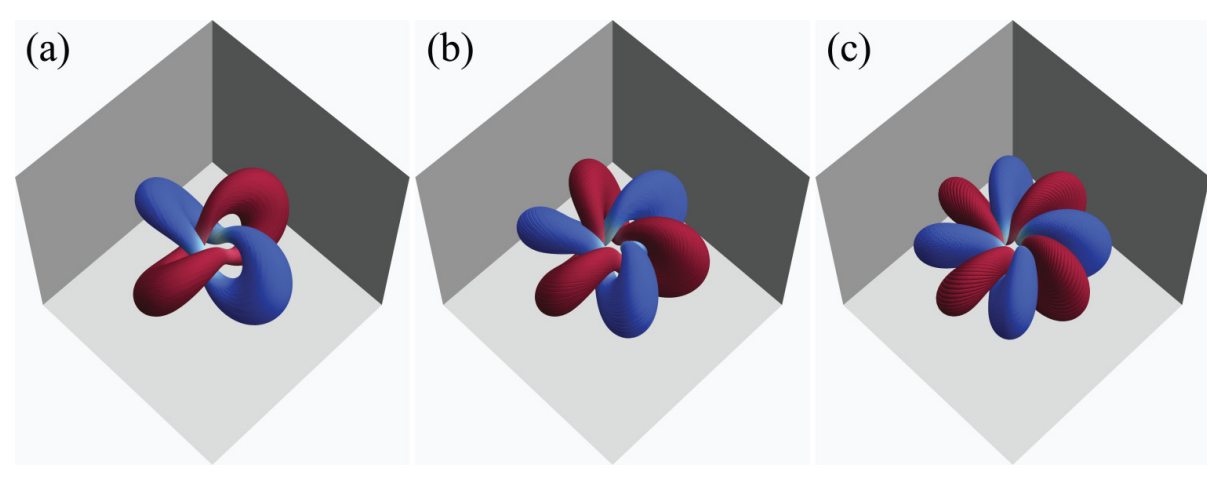

Figure 23: (Colour online) The magnetic surfaces for $n_{t}=1$ and (a) $n_{p}=2$, (b) $n_{p}=3$, and (c) $n_{p}=4$. Solutions with $n_{p} \neq 1$ have zero angular momentum and are therefore not stable solitons. The magnetic field lines in each lobe wind in opposite directions (represented by the red and blue surfaces).

where the positive velocity solution was taken. The conserved quantities $h_{m}$ and $\mathbf{L}$ fix the values of $R$ and $B_{0}$,

$$
\begin{aligned}
R & =\left[\frac{1}{8 \pi^{2} n_{t}\left(n_{t}+1\right)}\left(\frac{\mu_{0}}{\rho}\right) \frac{|L|^{2}}{h_{m}}\right]^{\frac{1}{4}}, \\
B_{0} & =2 n_{t}\left(n_{t}+1\right)\left(\frac{\rho}{\mu_{0}}\right)^{1 / 2} \frac{h_{m}}{|L|},
\end{aligned}
$$

thus inhibiting energy dissipation. This shows that the solution given in (243)(245) (and shown in Fig. 22) represents a class of topological solitons characterized by the positive integer $n_{t}$ for $n_{p}=1$.

For $n_{p} \neq 1$, the angular momentum for all $n_{t}$ is zero. Some examples of fields with $n_{t}=1$ and different $n_{p}$ values are shown in Fig. 23. The field lines fill two sets of linked surfaces. For a given pair of linked surfaces, the field in each lobe wraps around the surface in opposite directions. In Fig. 23 the red and blue surfaces wind in opposite directions. This means that the contribution to the angular momentum of the two field lines cancels. In this case the length scale is not fixed by the conserved quantities. The energy can therefore decrease by increasing the radius and the fields are not solitons.

For realistic plasma finite conductivity has to be considered. The effect of 
losses combined with plasma nonlinearities on the dynamics and plasma topology can be very complex because it will allow for magnetic field lines pinching off and reconnecting. Only a rough estimate of the soliton lifetime can be obtained by dividing the energy by $d \mathcal{E} / d t$, calculated before any energy dissipation [11]. Then a lower bound is obtained on the time it takes for the total energy to dissipate,

$$
\frac{d \mathcal{E}}{d t}=\frac{1}{\sigma} \int j^{2} d^{3} x=\left(3 n_{t}+7 n_{t}^{2}+5 n_{t}^{3}\right) \frac{\pi^{2} B_{0}^{2} R}{\mu_{0}^{2} \sigma} .
$$

The resulting lifetime is

$$
t_{n_{t}} \geq \frac{3 n_{t}}{3 n_{t}+7 n_{t}^{2}+5 n_{t}^{3}} \mu_{0} \sigma R^{2} .
$$

For higher $n_{t}$, the lifetime decreases although the helicity in (248) increases. This result is interesting as one would have expected from the results regarding flux tubes mentioned previously that the lifetime would increase with increasing helicity.

\section{Conclusions and outlook}

In this article we have reviewed the work on non-trivial topological solutions to Maxwell's equation in vacuum. An important example is the Hopf-Rañada field, which we have obtained using the Rañada formulation, the conformal transformation method, the Bateman construction and the twistor formalism.

This solution is characterized by the property that all the electric and magnetic field lines are linked loops. The helicity of this field is related to the Gauss linking number which coincides with the Hopf index, which is a topological invariant. We have addressed two new classes of solutions having the topology of the torus knots. One is a non-null class, where the electric and magnetic fields are not orthogonal. This class present the phenomena of exchanging helicity between the magnetic and electric field. The other class is a null one. We have reviewed the constructions and properties of both classes.

Maxwell's equations are invariant under conformal transformations. The group of conformal transformations contains the Poincaré group plus dilatations 

plasma.

A question that naturally arises is: can we generate linked and knotted forms of electromagnetic radiation in the laboratory? This question has been addressed in [17], concluding that indeed it should be possible, but very challenging, to generate these fields to a good approximation. To see this first consider the decomposition of the Hopf-Rañada field in vector spherical harmonics (VSPHs) $\mathbf{A}_{l m}^{T E}(k, \mathbf{r})$, labelled by angular-momentum integers $l \geq 1$ and $-l \leq m \leq l$, wavevector $k$ and polarization TE/TM (electric/magnetic field transverse to the radial direction) [103]. A general free-space vector potential can be expressed as:

$$
\mathbf{A}(\mathbf{r}, t)=\int \mathrm{d} k \sum_{l=1}^{\infty} \sum_{m=-l}^{m=l}\left[\alpha_{l m}^{T M}(k) \mathbf{A}_{l m}^{T M}(k, \mathbf{r})+\alpha_{l m}^{T E}(k) \mathbf{A}_{l m}^{T E}(k, \mathbf{r})\right] e^{-i \omega t}+\text { c.c. }
$$

The decomposition of the Hopf-Rañada field has the surprisingly simple form:

$$
\mathbf{A}_{\text {hopfion }}(\mathbf{r}, t)=\sqrt{\frac{4}{3 \pi}} \int \mathrm{d} k k^{3} e^{-k}\left[\mathbf{A}_{11}^{T E}(k, \mathbf{r})-i \mathbf{A}_{11}^{T M}(k, \mathbf{r})\right] e^{-i \omega t}+\text { c.c. }
$$

It only contains the $l=m=1$ spherical harmonics and has an energy spectrum $S(\omega) \approx \omega e^{-\omega}$. Furthermore the superposition $\mathbf{A}^{T E}-i \mathbf{A}^{T M}$ is an eigenstate of the curl operator with eigenvalue $+k$. Such eigenstates are known as Chandrasekhar-Kendall states for constant $k$ [104], and are examples of ${ }_{1230}$ force-free fields that play an important role in plasma physics and fluid dy- 
namics [98, 104]. The Hopf-Rañada field is therefore a pulsed version of the Chandrasekhar-Kendall states with a spectrum $S \omega e^{-\omega}$. The fact that $l=m$ is not too surprising since we are considering field configurations for which all the angular momentum is in the propagation direction. It has numerically been shown in [17] that a strongly focussed zeroth-order Gaussian beam with circular polarization converges towards a $l=m=1$ multipole field as the focusing angle increases towards $90^{\circ}$. In order to also produce the appropriate spectrum ultra short pulses of order one wavelength are required. Attosecond lasers can in principle produce approximately the require spectrum for visible light. Tightly focussing light with a very broad bandwidth is very challenging and perhaps the best way to proceed experimentally is to investigate longer wavelength EM radiation. The above decomposition in vector spherical harmonics also directly leads to a generalization of the Hopf-Rañada field by taking $l=m>1$. For the case $l=m=2$ it has been numerically shown that there exists a pair of core field lines that form two intertwined trefoil knots [17].

The creation and detections of knotted electromagnetic fields present a challenge from an experimental point of view and we only can foresee the potential applications of those fields, for example transporting the angular momentum which is carried by those fields, or taking advantages of the quasiparticle behaviour of such fields.

As a last remark we note that in the proof pages stage of this review three articles appeared online that generate more complex electromagnetic knots, such as cable knots [105, 106], and that describe collisions between knots [107].

\section{Acknowledgment}

We acknowledge J. W. Dalhuisen, A. Thompson and J. Swearngin, for valuable contributions. In particular sections 7, 8, 9, and Appendix B are based on material and figures that have been presented in their $\mathrm{PhD}$ and Master thesis and corresponding research articles.

We want to thank Prof. J. M. Montesinos Amilibia for useful discussions 


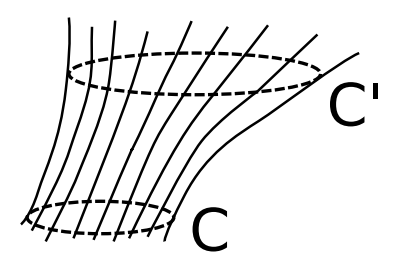

Figure 24: A magnetic tube forming a magnetic surface.

\section{Appendix A: The Gauss integral, the linking number and the helicity of flux tubes}

The value of the magnetic helicity is related to the topological configuration of the field lines. The simplest way to see the connection of topology and helicity is through the concept of a magnetic tube. Consider a small closed circular curve $C$. We define its magnetic tube [109] to be the set of all magnetic lines that intersect $C$ (Fig. 24). We will assume that that set of all the magnetic lines on $C$ forms a magnetic surface. The consequence is that the internal flux of any magnetic tube does not depend on the section through we compute it. We define the magnetic tube strength to be the flux through any section, so since 


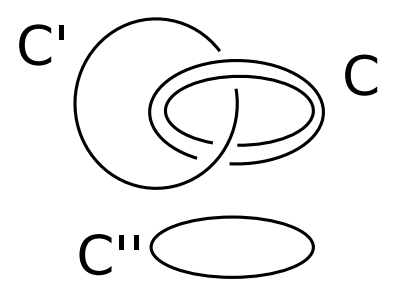

Figure 25: A filamental tube $C$ and two curves $C^{\prime}$ and $C^{\prime \prime}$. The Gauss linking number of the curve $C^{\prime}$ with respect to the filamental tube $C$ is $L\left(C, C^{\prime}\right)=1$. For the curve $C^{\prime \prime}$, the Gauss linking number is $L\left(C, C^{\prime \prime}\right)=0$.

$\mathbf{B}=\nabla \times \mathbf{A}$ we have

$$
\Phi_{C}=\int_{S_{1}} \mathbf{B} \cdot d \mathbf{S}=\oint_{C} \mathbf{A} \cdot d \mathbf{r}
$$

where $S_{1}$ is any surface such as $\partial S_{1}=C$. A filamental tube is a magnetic tube with infinitesimal section, but its strength is non-vanishing.

Suppose that a simply-connected domain $D$, on which a magnetic field $\mathbf{B}$ is defined. The helicity is gauge invariant, so we can take the Coulomb gauge for the vector potential $\nabla \cdot \mathbf{A}=0$, and using the Helmholtz theorem [110] we can write

$$
\mathbf{A}(\mathbf{r})=\frac{1}{4 \pi} \int_{D} \frac{\nabla^{\prime} \times \mathbf{B}\left(\mathbf{r}^{\prime}\right)}{R} d^{3} r^{\prime}
$$

being $\nabla^{\prime}=\left(\partial / \partial x^{\prime}, \ldots\right), \mathbf{R}=\mathbf{r}-\mathbf{r}^{\prime}$ and $R=\|\mathbf{R}\|$. Taking $\mathbf{B}=0$ in $\partial D$, it follows

$$
\mathbf{A}(\mathbf{r})=\frac{1}{4 \pi} \int_{D} \frac{\mathbf{B}\left(\mathbf{r}^{\prime}\right) \times \mathbf{R}}{R^{3}} d^{3} r^{\prime}
$$

Let us consider the particular case of a field created by a filamental tube $C$ inside the region $D$ and calculate the the circulation of the vector potential along a closed curve $C^{\prime}$ which does not have any common point with the tube, as we can see in Fig. 25,

$$
\Gamma_{C^{\prime}}=\oint_{C^{\prime}} \mathbf{A} \cdot d \mathbf{r} .
$$

As the field is created by the filamental tube, we can substitute $\mathbf{B}(\mathbf{r}) d r^{3}=$ $\mathbf{B} \cdot d \mathbf{S} d \mathbf{r}=\delta \Phi d \mathbf{r}$ in the expression for the potential (258), so we get

$$
\Gamma_{C^{\prime}}=\delta \Phi\left(\frac{1}{4 \pi} \oint_{C} \oint_{C^{\prime}}\left(d \mathbf{r} \times d \mathbf{r}^{\prime}\right) \cdot \frac{\mathbf{r}-\mathbf{r}^{\prime}}{\left\|\mathbf{r}-\mathbf{r}^{\prime}\right\|^{3}}\right) .
$$


The integral between brackets in (259) is known as the Gauss integral. The Gauss integral is an integer number $[111,112]$ also called the linking number. To see that we write

$$
\frac{1}{4 \pi} \oint_{C} \oint_{C^{\prime}}\left(d \mathbf{r} \times d \mathbf{r}^{\prime}\right) \cdot \frac{\mathbf{r}-\mathbf{r}^{\prime}}{\left\|\mathbf{r}-\mathbf{r}^{\prime}\right\|^{3}}=\frac{1}{4 \pi} \oint_{C} \oint_{C^{\prime}} \frac{\mathbf{r}-\mathbf{r}^{\prime}}{\left\|\mathbf{r}-\mathbf{r}^{\prime}\right\|^{3}} \times d \mathbf{r} \cdot d \mathbf{r}^{\prime},
$$

and using the Stokes theorem [112],

$$
\oint_{C} \frac{\mathbf{r}-\mathbf{r}^{\prime}}{\left\|\mathbf{r}-\mathbf{r}^{\prime}\right\|^{3}} \times d \mathbf{r}=-\int_{S} d \mathbf{S} \cdot \nabla_{\mathbf{r}} \frac{\mathbf{r}-\mathbf{r}^{\prime}}{\left\|\mathbf{r}-\mathbf{r}^{\prime}\right\|^{3}}=\nabla_{\mathbf{r}^{\prime}} \int_{S} d \mathbf{S} \cdot \frac{\mathbf{r}-\mathbf{r}^{\prime}}{\left\|\mathbf{r}-\mathbf{r}^{\prime}\right\|^{3}}
$$

The last integral is the solid angle of a surface whose boundary is the curve $C$ as seen from any point $\mathbf{r}^{\prime}$, so $(260)$ reads

$$
\frac{1}{4 \pi} \oint_{C} \oint_{C^{\prime}}\left(d \mathbf{r} \times d \mathbf{r}^{\prime}\right) \cdot \frac{\mathbf{r}-\mathbf{r}^{\prime}}{\left\|\mathbf{r}-\mathbf{r}^{\prime}\right\|^{3}}=\frac{1}{4 \pi} \oint_{C^{\prime}} \nabla_{\mathbf{r}^{\prime}} \Omega=\frac{1}{4 \pi}[\Omega]_{C^{\prime}}
$$

The change in the solid angle subtended by the curve $C$ when the curve $C^{\prime}$ is traveled all the way is an integer number times $4 \pi$, so

$$
L\left(C, C^{\prime}\right)=\frac{1}{4 \pi} \oint_{C} \oint_{C^{\prime}}\left(d \mathbf{r} \times d \mathbf{r}^{\prime}\right) \cdot \frac{\mathbf{r}-\mathbf{r}^{\prime}}{\left\|\mathbf{r}-\mathbf{r}^{\prime}\right\|^{3}}
$$

defines the linking number. In the cases shown in Fig. 25, $L\left(C, C^{\prime}\right)=1$ and $L\left(C, C^{\prime \prime}\right)=0$.

We will make now the connection with the helicity. The magnetic helicity in the region $D$ can be written as

$$
h_{m}=\frac{1}{2 c \mu_{0}} \frac{1}{4 \pi} \iint \mathbf{B}(\mathbf{r}) \times \mathbf{B}\left(\mathbf{r}^{\prime}\right) \cdot \frac{\mathbf{R}}{R^{3}} d^{3} r^{\prime} d^{3} r .
$$

Consider now the special situation in which $\mathbf{B}$ is zero except in two filamental tubes whose axes are some oriented, closed and non self-knotted curves $C$ y $C^{\prime}$ which can be linked (Fig. 26). The sections of the filamental tubes have zero limit, but as before they have non vanishing strengths $\delta \Phi, \delta \Phi^{\prime}$, respectively. Moreover, we must suppose that, along each filamental tube, the magnetic lines run parallel to $C$ and $C^{\prime}$ respectively. In this case, for each magnetic fields in (264) we can write $\mathbf{B}(\mathbf{r}) d r^{3}=\mathbf{B} \cdot d \mathbf{S} d \mathbf{r}=\delta \Phi d \mathbf{r}$ and we get

$$
h_{m}=\frac{1}{2 c \mu_{0}} \delta \Phi \delta \Phi^{\prime}\left(\frac{2}{4 \pi} \oint_{C} \oint_{C^{\prime}}\left(d \mathbf{r} \times d \mathbf{r}^{\prime}\right) \cdot \frac{\mathbf{r}-\mathbf{r}^{\prime}}{\left\|\mathbf{r}-\mathbf{r}^{\prime}\right\|^{3}}\right) .
$$




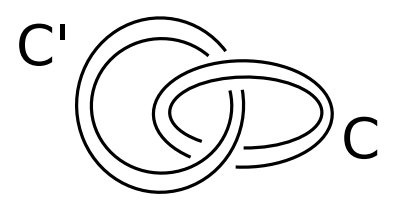

Figure 26: Oriented, linked and non self-knotted filamental tubes, without internal contribution to the helicity.

Note that the factor 2 enters as when integrating we have the two possibilities $\mathbf{r} \in C, \mathbf{r}^{\prime} \in C^{\prime}$ or $\mathbf{r}^{\prime} \in C, \mathbf{r} \in C^{\prime}$. We can see that the helicity of the two filamental tubes is proportional to the linking number of the tubes,

$$
h_{m}=\frac{1}{2 c \mu_{0}} 2 \delta \Phi \delta \Phi^{\prime} L\left(C, C^{\prime}\right) .
$$

This result was obtained by Moffatt [51]. In the computation, it is essential that each filamental tube has zero helicity by itself, and this condition is assured if the magnetic lines into each tube are not linked. In the case of having various closed filamental tubes, the total helicity will be

$$
h_{m}=\frac{1}{2 c \mu_{0}} \sum_{i} \sum_{j \neq i} L\left(C_{i}, C_{j}\right) \delta \Phi_{i} \delta \Phi_{j}
$$

$L\left(C_{i}, C_{j}\right)$ being the linking number of the tubes $C_{i}, C_{j}$.

The relationship between the linking number and the helicity of a selfknotted filamental tube $T$ has been studied by Berger and Field [87], and Moffatt and Ricca [16]. In this case we are looking for an expression for the helicity in a filamental tube of strength $\delta \Phi$, built around a closed magnetic line $C$ as before, but as the tube is self-knotted (see Fig. 27), then its axis $C$ has a non-vanishing torsion, and the twisting of the magnetic lines inside the tube must be taken into account.

It can be shown that the magnetic helicity of a self-linked filamental tube reads

$$
h_{m}=\frac{1}{2 c \mu_{0}}(\delta \Phi)^{2}[\mathcal{W}(C)+\mathcal{T} w(C)]=\frac{1}{2 c \mu_{0}}(\delta \Phi)^{2}\left[\mathcal{W}(C)+\mathcal{T}(C)+\mathcal{T}_{0}\left(C, C_{m}\right)\right]
$$




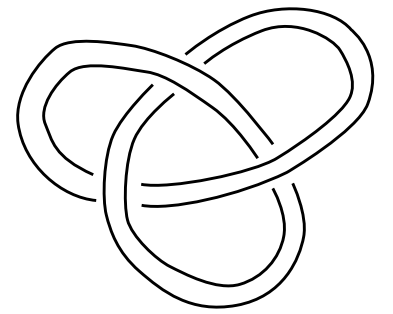

Figure 27: A filamental tube built around the trefoil knot.

The quantity $\mathcal{W}(C)$ is the spatial writhing number which is real number defined by the limiting form of the Gauss integral (265).

$$
\mathcal{W}(C)=\frac{1}{4 \pi} \oint_{C} \oint_{C} \frac{\left(d \mathbf{r} \times d \mathbf{r}^{\prime}\right) \cdot\left(\mathbf{r}-\mathbf{r}^{\prime}\right)}{\left\|\mathbf{r}-\mathbf{r}^{\prime}\right\|^{3}} .
$$

For the interpretation of $\mathcal{W}(C)$ see $[16,113]$.

The quantity $\mathcal{T} w$ is the total twist number of the ribbon with boundaries the axis curve $C$ and any other field line on the filamental tube. It was introduced by Fuller [114]. The total twist number of a ribbon is defined as the number of times that the ribbon revolves around its own axis

$$
\mathcal{T} w(C)=\frac{1}{2 \pi} \oint_{C} \frac{d \hat{\mathbf{r}}}{d s} \times \hat{\mathbf{r}} \cdot \mathbf{t} d s=\frac{1}{2 \pi} \oint_{C}\left(\tau+\frac{d \theta}{d s}\right) d s=\mathcal{T}(C)+\frac{1}{2 \pi}[\theta]_{C},
$$

where $\mathbf{t}$ is the unitary tangent and $s$ the arc parameter of the axial curve $C$. The second equality is obtained using Frenet's relations, $\tau$ is the torsion and

$$
\mathcal{T}(C)=\frac{1}{2 \pi} \oint_{C} \tau(s) d s
$$

is the total torsion of the axial curve $C$ normalized by $(2 \pi)^{-1}$. Finally

$$
\mathcal{T}_{0}\left(C, C_{m}\right)=\frac{1}{2 \pi}[\theta]_{C}=N,
$$

which is an integer number giving the number of times that the boundary $C_{m}$ of the ribbon, given by a field line on the tube, rounds the other boundary $C$, which is in this case the axis of the tube.

None of the numbers $\mathcal{W}, \mathcal{T}$ and $\mathcal{T}_{0}$ is a topological invariant, and only the last one is an integer number. However Calugareanu proved that their sum

$$
\mathcal{W}(C)+\mathcal{T}(C)+\mathcal{T}_{0}\left(C, C_{m}\right)=L\left(C, C_{m}\right) .
$$


is a topological invariant, equal to the Gauss linking number of the axis $C$ and any magnetic line $C_{m}$ that rounds the surface of the tube $T$. That is the Calugareanu's theorem [115]. Using this theorem, we conclude that the magnetic helicity of a filamental tube $T$ whose axis is a closed, self-knotted curve $C$, is given by

$$
h_{m}=\frac{1}{2 c \mu_{0}}(\delta \Phi)^{2} L\left(C, C_{m}\right)
$$

where $L\left(C, C_{m}\right)$ is the linking number of the axis $C$ and any magnetic line $C_{m}$ that rounds the surface of the tube $T$. In conclusion, the helicity of a divergenceless vector field is a measure of the linking number of the field lines.

\section{Appendix B: A brief survey of twistor theory}

The starting point for the twistor formalism is the spinor formalism. Consider a 4 -vector $x^{a}=(t, x, y, z)$ and represent it by a 2 by 2 complex matrix $x^{A A^{\prime}}$, with $A=0,1$ and $A^{\prime}=0,1$ in the following way,

$$
x^{A A^{\prime}}=\frac{1}{\sqrt{2}}\left(\begin{array}{cc}
t+z & x+i y \\
x-i y & t-z
\end{array}\right) .
$$

The advantage of this notation is that the determinant of $x^{A A^{\prime}}$ is the 4-interval $t^{2}-x^{2}-y^{2}-z^{2}$. Therefore a matrix transformation of $x^{A A^{\prime}} \rightarrow L x^{A A^{\prime}} L^{\dagger}$, with $L$ an element of $S L(2, C)$ (the group of complex $2 \times 2$ matrices with determinant 1 ), and $L^{\dagger}$ its transposed and complex conjugated matrix, is associated with a Lorentz transformation. Clearly $S L(2, C)$ is 6 (real) dimensional (4 complex numbers and two constraints $\operatorname{det}(L)=1)$ and it is 2 to 1 isomoprphic to $S O(1,3)$, the proper Lorentz group. It is instructive to check that for example

$$
L=\left(\begin{array}{cc}
e^{i \psi} & 0 \\
0 & e^{-i \psi}
\end{array}\right)
$$

corresponds to a rotation over $2 \psi$ around the $z$-axis. Note that $\psi=\pi$ is minus the identity matrix and corresponds to a $2 \pi$ rotation around the $z$-axis that leaves $x^{A A^{\prime}}$ unchanged. Having made a connection between $S L(2, C)$ and Lorentz transformation of 2 by 2 matrices representing space time points, it 
is natural to have the element of $S L(2, C)$ act on a two (complex) component vectors $\pi^{A}=\left(\pi^{0}, \pi^{1}\right)$. These are spinors and they do acquire a minus sign for elements of $S L(2, C)$ that represent a $2 \pi$ rotation in space.

If we restrict our attention to points on the light cone $\left(t^{2}-x^{2}-y^{2}-z^{2}=0\right.$ or equivalently $\operatorname{det}\left(x^{A A^{\prime}}\right)=0$ ) we see that the components of a single spinor $\pi^{A}=\left(\pi^{0}, \pi^{1}\right)$ and their complex conjugate values suffice to defines such points

$$
x^{A A^{\prime}}=\left(\begin{array}{cc}
\bar{\pi}^{0} \pi^{0} & \bar{\pi}^{0} \pi^{1} \\
\bar{\pi}^{1} \pi^{0} & \bar{\pi}^{1} \pi^{1}
\end{array}\right) .
$$
spin space is two dimensional any anti-symmetrization over more than 2 indices has to be zero.

To get familiar with the spinor notation consider the spinor equivalent of a general valence 2 tensor $T_{a b} \rightarrow T_{A A^{\prime} B B^{\prime}}=T_{A B A^{\prime} B^{\prime}}$ (the order of primed and 
unprimed indices is irrelevant). Using symmetrization and anti-symmetrization on the unprimed pair we obtain

$$
T_{A B A^{\prime} B^{\prime}}=T_{(A B) A^{\prime} B^{\prime}}+T_{[A B] A^{\prime} B^{\prime}}=T_{(A B) A^{\prime} B^{\prime}}+\frac{1}{2} \epsilon_{A B} T_{C}{ }_{A^{\prime} B^{\prime}} .
$$

In the last step we use the fact that an antisymmetric pair of indices can be removed as an $\epsilon_{A B}$ with a contraction. In this sense, only symmetric spinors count and any spinor can be written in terms of symmetric spinors and the special spinors $\epsilon_{A B}, \epsilon_{A^{\prime} B^{\prime}}$ and their inverses. Using also symmetrization and anti-symmetrization on the primed pair we obtain

$$
\begin{aligned}
T_{A B A^{\prime} B^{\prime}}=T_{(A B)\left(A^{\prime} B^{\prime}\right)}+ & \frac{1}{2} \epsilon_{A^{\prime} B^{\prime}} T_{(A B) C^{\prime}}{ }^{C^{\prime}}+\frac{1}{2} \epsilon_{A B} T_{C}{ }_{\left(A^{\prime} B^{\prime}\right)} \\
& +\frac{1}{4} \epsilon_{A B} \epsilon_{A^{\prime} B^{\prime}} T_{C C^{\prime}}{ }^{C C^{\prime}}
\end{aligned}
$$

The first term is the trace free symmetric part of $T_{a b}$ and the last term is the symmetric term $\frac{1}{4} \eta_{a b} T_{c}^{c}$. The middle two terms are anti-symmetric in $a$ and $b$. If we now restrict our attention to the important case of a real bivector (antisymmetric in $a$ and $b$ ) which we call $F_{a b}$ (for example the Faraday tensor in electromagnetism or the relativistic total angular momentum tensor) one concludes that

$$
F_{a b} \rightarrow F_{A A^{\prime} B B^{\prime}}=\Phi_{A B} \epsilon_{A^{\prime} B^{\prime}}+\bar{\Phi}_{A^{\prime} B^{\prime}} \epsilon_{A B}
$$

where $\Phi_{A B}$ is symmetric. If we now consider the dual of the bivector $F_{a b}$ we have

$$
{ }^{*} F_{a b}=\frac{1}{2} \epsilon_{a b}^{c d} F_{c d}=-i \Phi_{A B} \epsilon_{A^{\prime} B^{\prime}}+i \bar{\Phi}_{A^{\prime} B^{\prime}} \epsilon_{A B} .
$$

The self-dual part of $F_{a b}\left({ }^{*} W_{a b}=i W_{a b}\right)$ is now given by $\bar{\Phi}_{A^{\prime} B^{\prime}} \epsilon_{A B}$, and the anti-self-dual part of $F_{a b}\left({ }^{*} W_{a b}=-i W_{a b}\right)$ is $\Phi_{A B} \epsilon_{A^{\prime} B^{\prime}}$.

Since $\Phi_{A B}$ in (281) is symmetric it can be written as $\alpha_{(A} \beta_{B)}$. In other words $\Phi_{A B}$ can be factorized into two spinors (defining two principal null directions via the corresponding flag poles). Combining the above spinor manipulations we now have arrived at the following spinor notation for a real bivector

$$
F_{a b} \rightarrow F_{A A^{\prime} B B^{\prime}}=i \omega_{(A} \bar{\pi}_{B)} \epsilon_{A^{\prime} B^{\prime}}+\text { c.c. },
$$


where the first term is the self-dual part of $F$ and the second term the anti-selfdual part.

The source-free Maxwell equations in terms of the Faraday tensor (a real bivector) $F_{a b}$ are

$$
\nabla_{[a} F_{b c]}=0 ; \quad \nabla^{a} F_{a b}=0 .
$$

In terms of the anti-self-dual part of $F_{a b}, W_{a b}=F_{a b}+i^{*} F_{a b}$, the Maxwell equations are compactly written as

$$
\nabla^{a} W_{a b}=0
$$

In spinor terms this becomes

$$
\nabla^{A A^{\prime}} \psi_{A B} \epsilon_{A^{\prime} B^{\prime}}=\nabla_{A^{\prime}}^{A} \psi_{A B}=0
$$

with $\psi_{A B}$ a symmetric spinor, and

$$
\nabla_{A^{\prime}}{ }^{A}=\frac{1}{\sqrt{2}}\left(\begin{array}{cc}
-\partial_{x}-i \partial_{y} & -\partial_{t}+\partial_{z} \\
\partial_{t}+\partial_{z} & \partial_{x}-i \partial_{y}
\end{array}\right)
$$

In a similar way the zero rest mass free field equations of arbitrary half integer spin can be formulated in terms of symmetric spinor fields. In particular 1325 the equations

$$
\begin{aligned}
\nabla_{A^{\prime}}{ }^{A} \psi_{A} & =0, \\
\nabla_{A^{\prime}}{ }^{A} \psi_{A B} & =0, \\
\nabla_{A^{\prime}}{ }^{A} \psi_{A B C D} & =0,
\end{aligned}
$$

are the spin $\frac{1}{2}$ massless neutrino equation, the spin 1 Maxwell vacuum equations, and the spin 2 linearized Einstein vacuum equations, respectively.

In the following we review how twistor theory allows for solving these equations such that linked and knotted field solutions emerge as the most natural solutions.

One can think of twistor space $\mathbb{T}$ as the total momentum space for massless particles $[12,79]$ in which the linear and angular momenta are combined 
into a single object called a twistor $Z^{\alpha}$, an object with 4 complex variables $(\alpha=0,1,2,3)$. This is accomplished by realizing that the combined linear and angular momentum possess two spinorial degrees of freedom denoted by $\omega^{A}$ and $\pi_{A^{\prime}}$. Since the linear 4-momentum of a massless field $p^{a}$ is light-like it can be decomposed as the flagpole of a first spinor $\pi_{A^{\prime}}$,

$$
p^{A A^{\prime}}=\bar{\pi}^{A} \pi^{A^{\prime}} .
$$

In general the center of mass Lorentz boosts and the orbital angular momentum of a rotating object are combined into a four dimensional bivector $M=X \wedge P$ which in components reads $M^{\alpha \beta}=X^{\alpha} P^{\beta}-X^{\alpha} P^{\beta}$, with $X^{\alpha}$ the 4 -position and $P^{\alpha}$ the 4-momentum. The second spinor $\omega^{A}$ is related to the relativistic angular momentum tensor $M^{a b}$ in that $i \omega^{(A} \bar{\pi}^{B)} \epsilon^{A^{\prime} B^{\prime}}$ and its conjugate represent the self-dual (SD) and anti-self-dual (ASD) parts (see (283)) of $M^{a b}$ respectively,

$$
M^{a b}=M^{A A^{\prime} B B^{\prime}}=i \omega^{(A} \bar{\pi}^{B)} \epsilon^{A^{\prime} B^{\prime}}+\text { c.c. }
$$

The pair $\left(\omega^{A}, \pi_{A^{\prime}}\right)$ is a twistor $Z^{\alpha}$, with components

$$
\begin{aligned}
\left(Z^{0}, Z^{1}\right) & \equiv\left(\omega^{0}, \omega^{1}\right) \\
\left(Z^{2}, Z^{3}\right) & \equiv\left(\pi_{0^{\prime}}, \pi_{1^{\prime}}\right) .
\end{aligned}
$$

The dependence of the momentum structure $\left(M^{a b}, p^{c}\right)$ on the origin induces a position dependence in $\left(\omega^{A}, \pi_{A^{\prime}}\right)$, making them spinor fields on space-time defined by

$$
\begin{aligned}
\pi_{A^{\prime}}(x) & =\pi_{A^{\prime}}, \\
\omega^{A}(x) & =\omega^{A}-i x^{A A^{\prime}} \pi_{A^{\prime}} .
\end{aligned}
$$

In order to define a norm in twistor space we have to introduce dual twistors given by $\bar{Z}_{\alpha}=\left(\bar{\pi}_{A}, \bar{\omega}^{A^{\prime}}\right)$. With this definition it follows that the norm in twistor space is twice the helicity $\mathfrak{h}$ associate to the massless field configuration represented by a given twistor $Z^{\alpha}$,

$$
\mathfrak{h}=\frac{1}{2} Z^{\alpha} \bar{Z}_{\alpha} .
$$




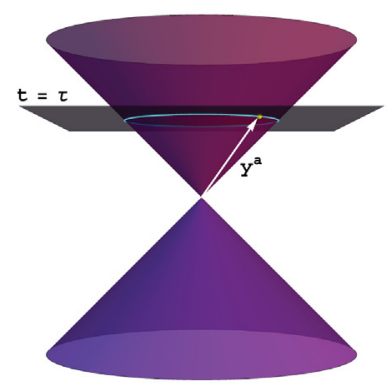

b

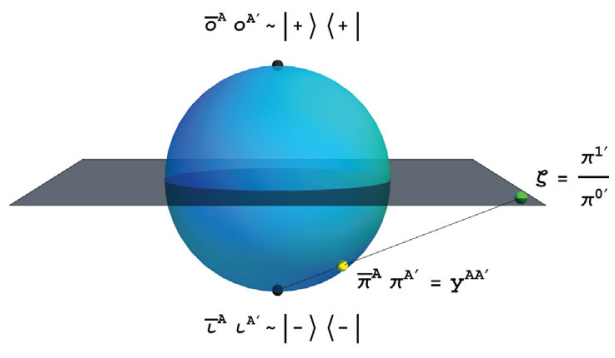

Figure 28: (Colour online) The flagpole relation is the central relation in the spin geometry of $M$. Here we see that to every 2-spinor $\pi^{A^{\prime}}$ there corresponds a light-like 4-vector $y^{a}$ called its flagpole. a When the lightcone of the origin (violet) is intersected with the space-like hyperplane $t=\tau$ (dark grey), we obtain the Minkowski space representation of an expanding sphere of light emitted at the origin, called the celestial sphere (blue). Any light-like 4-vector $y^{a}$ (white) may be represented by the unique point where it meets this sphere (yellow). $\mathbf{b}$ Analogous to the pure state representation of the Bloch sphere, the celestial sphere may be put in 1-1 correspondence with the set of $2 \times 2$ Hermitian matrices with trace equal to $\sqrt{2} \tau$. The 2-spinor $\pi^{A^{\prime}}$ is taken as a homogenous coordinate on the complex plane (light grey) of the sphere $\zeta=\pi^{1^{\prime}} / \pi^{0^{\prime}}$ (green). The flagpole of $\pi^{A^{\prime}}$ is then identified with the 4-vector $y^{A A^{\prime}}$ written in flagpole form as a $2 \times 2$ Hermitian matrix.

Helicity, the twistor norm, is invariant under conformal transformations and therefore it plays a similar role as the 4-interval in Minkowski space-time.

Helicity provides for a natural geometric partition of twistor space $\mathbb{T}$ into three parts, $\mathbb{T}^{+}, \mathbb{T}^{-}$, and $\mathbb{N}$, according to whether the helicity is positive, negative, or zero. Twistors from $\mathbb{N}$ are said to be null, otherwise they are non-null.

Twistor geometry in $M$ can be defined by the vanishing of $\omega^{A}(x)$ in (295), yielding the incidence relation

$$
\omega^{A}=i x^{A A^{\prime}} \pi_{A^{\prime}}
$$

This relation can be solved for Hermitian $x^{A A^{\prime}}$, and thus points in $M$, only 

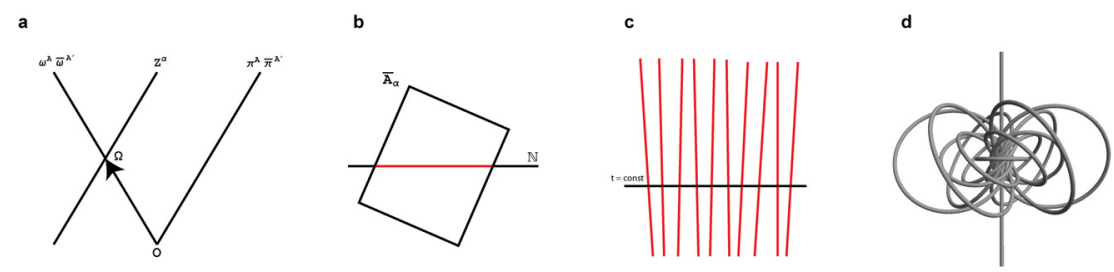

Figure 29: (Colour online) A null twistor $Z^{\alpha}$ corresponds to a light-like world-line in $M$. A non-null twistor $A^{\alpha}$ possesses only an indirect correspondence in $M$ constructed via the direct correspondence for null twistors. a If $Z^{\alpha}=\left(\omega^{A}, \pi_{A^{\prime}}\right)$ is a null twistor, then it corresponds to a light-like world-line (null geodesic) in $M$, parallel to the flagpol of $\pi_{A^{\prime}}$, which meets the lightcone of the origin along the light-like ray parallel to the flagpole of $\omega^{A}$. Here $\Omega=x^{A A^{\prime}}(0)$. b The dual of a non-null twistor $\bar{A}_{\alpha}$ is a plane in $\mathbb{T}$ which is uniquely defined by its intersection with $\mathbb{N}$ (red). c The Minkowski space representation of the non-null twistor $\bar{A}_{\alpha}$ wherein each of the null twistors in the intersection with $\mathbb{N}$ appear as the null geodesics in $M$ which comprise the Robinson congruence. $\mathbf{d}$ Viewed on a hyperplane of constant time, the Robinson congruence defines a vector field whose integral curves are the fibers of a Hopf map projected down stereographically onto the hyperplane.

when $Z^{\alpha}$ is null. In that case the solution is

$$
x^{A A^{\prime}}(r)=\frac{\omega^{A} \bar{\omega}^{A^{\prime}}}{i \bar{\omega}^{B^{\prime}} \pi_{B^{\prime}}}+r \bar{\pi}^{A} \pi^{A^{\prime}}
$$

for arbitrary real $r$. The geometry of equation (298) is shown in Fig. 29 and represents a light-like worldline in $M$ parallel to the flagpole of $\pi_{A^{\prime}}$, which meets the lightcone of the origin along the flagpole of $\omega^{A}$ at the point $x^{A A^{\prime}}(0)$.

For non-null twistors, (297) possesses no Hermitian solutions, and thus its direct geometric interpretation is in terms of complexified Minkowski space. However, as shown in Fig. 29, we can obtain an indirect geometric correspondence by exploiting the more direct relation for null twistors. If $A^{\alpha}$ is non-null then its dual $\bar{A}_{\alpha}$ is a plane in $\mathbb{T}$. This plane is uniquely defined by its intersection with $\mathbb{N}$. By (298), each point in this intersection represents a null geodesic. The set of all null twistors in the intersection corresponds to a space-filling set of null geodesics in $M$ called the Robinson congruence. If we project the tangent vector field of one of these congruences onto a hyperplane of constant $t$ then we find that it forms the tangent vector field of a Hopf fibration. The integral curves of 


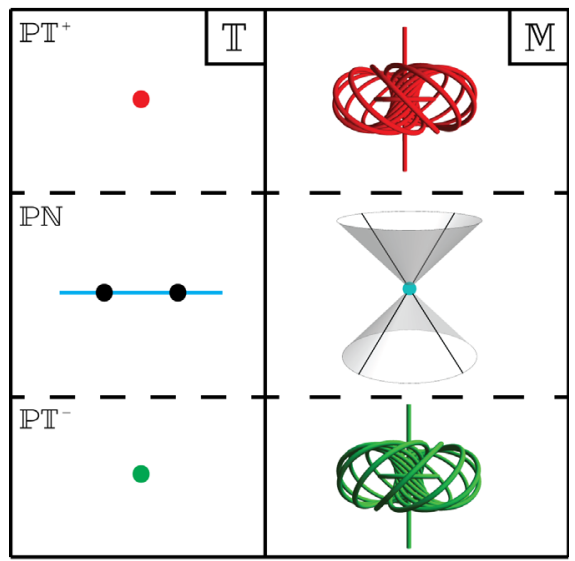

Figure 30: (Colour online) A summary of the geometric correspondences in terms of $\mathbb{P T}$ and $M$. A point in either $\mathbb{P T}^{+}$(red) or $\mathbb{P T}^{-}$(green) corresponds to a Robinson congruence in $M$. An entire $\mathbb{C P}^{1}$ (celestial sphere) in $\mathbb{P N}$ (blue) corresponds to a point in $M$. Any two points which lie on a $\mathbb{C P}^{1}$ in $\mathbb{P N}$ correspond to light-like world-lines which lie on the lightcone of the point corresponding to that $\mathbb{C P}^{1}$.

the vector field are a special family of circles which lie on a set of space-filling nested tori. Each circle, called a Villarceau circle, is linked with every other one exactly once, and the entire structure propagates with the speed of light along its axis of symmetry without deformation. In fact, it was the Hopf structure of the Robinson congruence which inspired Penrose with the name twistor.

The momentum correspondences (291) and (292) clearly possess $U(1)$ invariance. Yet the geometric correspondence defined by the incidence relation (297) is projective. That is, a twistor $Z^{\alpha}$ multiplied by any non-zero complex number corresponds to the same object in $M$ as $Z^{\alpha}$ itself. This leads us to consider as most fundamental the projective twistor space $\mathbb{P} \mathbb{T}$ defined as $\mathbb{T}$ under the equivalence relation $Z^{\alpha} \sim \lambda Z^{\alpha}$ for $\lambda \neq 0$. The partitions of $\mathbb{T}$ naturally extend to partitions of $\mathbb{P T}$, defnoted by $\mathbb{P T}^{+}, \mathbb{P T}^{-}$, and $\mathbb{P N}$. The geometric correspondences are most succinctly characterized in terms of the partitions of $\mathbb{P T}$, as shown in Fig. 30. 


\section{Twistor Quantization and Helicity Eigenfunctions}

Now we will convert helicity, as given in (296), into an operator, via the standard quantization procedure, and show that this operator and its eigenfunctions and eigenvalues are related to the massless spin- $N$ field equations and their solutions.

The canonical commutation relations

$$
\left[x^{a}, x^{b}\right]=0, \quad\left[p^{a}, p^{b}\right]=0 \quad\left[p_{b}, x^{a}\right]=i \hbar \delta_{b}^{a}
$$

lead to a choice of representation: either the position representation

$$
\left(\hat{x}^{a}, \hat{p}_{b}\right)=\left(x^{a},-i \hbar \frac{\partial}{\partial x^{b}}\right)
$$

or the momentum representation

$$
\left(\hat{x}^{a}, \hat{p}_{b}\right)=\left(i \hbar \frac{\partial}{\partial p_{a}}, p_{b}\right) .
$$

The operators act on wave functions $\Psi(x)$, or $\Psi(p)$ respectively. Since a twistor is directly related to position and momentum the above commutation relations translate into the twistor commutation relations

$$
\left[Z^{\alpha}, Z^{\beta}\right]=0, \quad\left[\bar{Z}_{\alpha}, \bar{Z}_{\beta}\right]=0, \quad\left[Z^{\alpha}, \bar{Z}_{\beta}\right]=\delta_{\beta}^{\alpha},
$$

leading to a choice of representation

$$
\begin{aligned}
\left(\hat{Z}^{\alpha}, \hat{\bar{Z}}_{\beta}\right) & =\left(Z^{\alpha},-\frac{\partial}{\partial Z^{\beta}}\right), \\
\text { or } \quad\left(\hat{Z}^{\alpha}, \hat{\bar{Z}}_{\beta}\right) & =\left(\frac{\partial}{\partial \bar{Z}_{\alpha}}, \bar{Z}_{\beta}\right) .
\end{aligned}
$$

The operators act on functions $f\left(Z^{\alpha}\right)$, and $f\left(\bar{Z}^{\alpha}\right)$ respectively. Taking for example the first choice (the $Z^{\alpha}$ representation) implies that $\frac{\partial}{\partial \bar{Z}} f(Z)=0$ what in turn implies that $f$ has to be a holomorphic (complex analytic) function in $Z^{\alpha}$ 1375 (4 complex variables) [13].

For massless free fields, helicity is the only quantum number and so the dynamics is entirely determined by the eigenfunctions of the helicity operator. 
Polarizing to avoid factor ordering issues and converting to our chosen representation, we have that

$$
\mathfrak{h}=\frac{1}{4}\left(Z^{\alpha} \bar{Z}_{\alpha}+\bar{Z}_{\alpha} Z^{\alpha}\right)=-\frac{1}{2}\left(Z^{\alpha} \frac{\partial}{\partial Z^{\alpha}}+2\right) .
$$

The operator $z \frac{\partial}{\partial z}$ is known as the Euler homogeneity operator and its eigenfunctions satisfy

$$
\begin{aligned}
z \frac{\partial}{\partial z} f(z) & =n f(z), \\
f(t z) & =t^{n} f(z),
\end{aligned}
$$

with $n$ an integer. We recognize (305) as representing a shifted Euler homogeneity operator on twistor space, and thus its eigenfunctions are homogeneous twistor functions where the relation between the homogeneity $n$ and the eigenvalue $h$ is given by

$$
n=-2 h-2 \text {. }
$$

If we want to consider say massless fields with helicity $h=1$ we have to use twistor functions of homogeneity $n=-4$. All we then have to do is to "translate" this twistor function into a field in Minkowski space. This is accomplices by the Penrose transform.

\section{The Penrose Transform}

The Penrose transform is an integral transform, dependent on helicity, which maps the holomorphic eigenfunctions of the helicity operator onto self dual (SD) or and anti self dual (ASD) solutions of the spin- $N$ field equations on $M$ [13]. Since we are interested only in classical fields, and thus fields whose SD and ASD components are conjugate, we require only the Penrose transform for positive helicity.

Let's introduce the Penrose transform for a simple case namely a $h=0$ field for which the twistor function must have homogeneity $n=-2$. The simplest of such functions would be

$$
f\left(Z^{\alpha}\right)=\frac{1}{\left(\bar{A}_{\alpha} Z^{\alpha}\right)^{2}}
$$


where $\bar{A}_{\alpha}=\left(\mu_{A}, \lambda^{A^{\prime}}\right)$ is a constant dual twistor. As will become clear below, this function will result in a trivial solution (field is zero) because it has only a single (doubly degenerate) pole. Next consider

$$
f\left(Z^{\alpha}\right)=\frac{1}{\left(\bar{A}_{\alpha} Z^{\alpha}\right)\left(\bar{B}_{\beta} Z^{\beta}\right)},
$$

where both $\bar{A}_{\alpha}$ and $\bar{B}_{\beta}$ are constant dual twistors. We aim to find an expressions for the corresponding zero-rest-mass field at a given point $x^{A A^{\prime}}$ in (complexified) Minkowski space. Through the incidence relation (297) we need to restrict $Z^{\alpha}=\left(\omega^{A}, \pi_{A^{\prime}}\right)$ to $L_{x}$ what implies $\omega^{A}=i x^{A A^{\prime}} \pi_{A^{\prime}}$. Let $\bar{A}_{\alpha}=\left(\mu_{A}, \lambda^{A^{\prime}}\right)$, $\bar{B}_{\alpha}=\left(\sigma_{A}, \psi^{A^{\prime}}\right)$ be the dual twistors then,

$$
\begin{aligned}
\bar{A}_{\alpha} Z^{\alpha} & =i \mu_{A} x^{A A^{\prime}} \pi_{A^{\prime}}+\lambda^{A^{\prime}} \pi_{A^{\prime}} \\
& \equiv \mathcal{A}^{A^{\prime}} \pi_{A^{\prime}} \\
\bar{B}_{\beta} Z^{\beta} & =i \sigma_{B} x^{B B^{\prime}} \pi_{B^{\prime}}+\psi^{B^{\prime}} \pi_{B^{\prime}} \\
& \equiv \mathcal{B}^{A^{\prime}} \pi_{B^{\prime}} .
\end{aligned}
$$

When the point $x^{A A^{\prime}}$ is in real Minkowski space (which we will be interested in here) $Z^{\alpha}$ is restricted to all twistors that correspond to null geodesics going through $x^{A A^{\prime}}$. Each such twistor corresponds to a point on the celestial sphere of $x^{A A^{\prime}}$.

The spin 0 field at $x$ is now given by the contour integral on this celestial sphere $\left(\mathbb{C P}^{1}\right)$ :

$$
\varphi(x)=\frac{1}{2 \pi i} \oint_{\Gamma} \frac{1}{\left(\mathcal{A}^{A^{\prime}} \pi_{A^{\prime}}\right)\left(\mathcal{B}^{A^{\prime}} \pi_{A^{\prime}}\right)} \pi_{C^{\prime}} d \pi^{C^{\prime}}
$$

Note that

$$
\begin{aligned}
\pi_{C^{\prime}} d \pi^{C^{\prime}} & =\pi_{C^{\prime}} d \pi_{D^{\prime}} \epsilon^{D^{\prime} C^{\prime}} \\
& =\pi_{0^{\prime}} d \pi_{1^{\prime}}-\pi_{1^{\prime}} d \pi_{0^{\prime}} \\
& =\left(\pi_{0^{\prime}}\right)^{2} d\left(\frac{\pi_{1^{\prime}}}{\pi_{0^{\prime}}}\right),
\end{aligned}
$$

and introducing the canonical spin bases $\left\{o_{A^{\prime}}, \iota_{A^{\prime}}\right\}$ into the primed spin space 
$S^{\prime}$ one obtains

$$
\begin{aligned}
\pi_{A^{\prime}} & =\pi_{0^{\prime}} o_{A^{\prime}}+\pi_{1^{\prime}} \iota_{A^{\prime}} \\
& =\pi_{0^{\prime}}\left(o_{A^{\prime}}+\left(\frac{\pi_{1^{\prime}}}{\pi_{0^{\prime}}}\right) \iota_{A^{\prime}}\right) .
\end{aligned}
$$

Together with the relations

$$
\begin{aligned}
& \frac{1}{\pi_{0^{\prime}}} \mathcal{A}^{A^{\prime}} \pi_{A^{\prime}}=\mathcal{A}^{0^{\prime}}+\mathcal{A}^{1^{\prime}}\left(\frac{\pi_{1^{\prime}}}{\pi_{0^{\prime}}}\right), \\
& \frac{1}{\pi_{0^{\prime}}} \mathcal{B}^{A^{\prime}} \pi_{A^{\prime}}=\mathcal{B}^{0^{\prime}}+\mathcal{B}^{1^{\prime}}\left(\frac{\pi_{1^{\prime}}}{\pi_{0^{\prime}}}\right),
\end{aligned}
$$

this indeed implies that (308) becomes an integral over the celestial sphere $\mathbb{C P}^{1}$. Thus,

$$
\begin{aligned}
\varphi(x) & =\frac{1}{2 \pi i} \oint_{\Gamma} \frac{1}{\left(\mathcal{A}^{0^{\prime}}+\mathcal{A}^{1^{\prime}}\left(\frac{\pi_{1^{\prime}}}{\pi_{0^{\prime}}}\right)\right)\left(\mathcal{B}^{0^{\prime}}+\mathcal{B}^{1^{\prime}}\left(\frac{\pi_{1^{\prime}}}{\pi_{0^{\prime}}}\right)\right)} d\left(\frac{\pi_{1^{\prime}}}{\pi_{0^{\prime}}}\right) \\
& =\frac{1}{2 \pi i \mathcal{A}^{1^{\prime}} \mathcal{B}^{1^{\prime}}} \oint_{\Gamma} \frac{1}{(\mu+\zeta)(\nu+\zeta)} d \zeta .
\end{aligned}
$$

where $\zeta=\pi_{1^{\prime}} / \pi_{0^{\prime}}, \mu=\mathcal{A}^{0^{\prime}} / \mathcal{A}^{1^{\prime}}$, and $\nu=\mathcal{B}^{0^{\prime}} / \mathcal{B}^{1^{\prime}}$ represent the projective coordinate and poles respectively. Since the contour integral is over a sphere the contour can always be contracted to a point if there is only a single (possibly degenerate) pole as is the case of (307). Only if there are two (or more) distinct poles, that is $\mu \neq \nu$ for the case discussed here, the integral will be nonzero.

After the variable substitutions, the integral is straightforward. Taking $\Gamma$ to enclose $-\mu$ we have

$$
\begin{aligned}
\varphi(x) & =\frac{1}{\mathcal{A}^{1^{\prime}} \mathcal{B}^{1^{\prime}}} \operatorname{Res}_{\zeta=-\mu} \frac{1}{(\mu+\zeta)(\nu+\zeta)} \\
& =\frac{1}{\mathcal{A}^{1^{\prime} \mathcal{B}^{1^{\prime}}(\nu-\mu)}} \\
& =\frac{2}{\left(\mu_{B} \sigma^{B}\left(x^{a}-y^{a}\right)\left(x_{a}-y_{a}\right)\right)},
\end{aligned}
$$

where the point $y$ is given by

$$
y^{A A^{\prime}}=i \frac{\sigma^{A} \lambda^{A^{\prime}}-\mu^{A} \psi^{A^{\prime}}}{\mu_{B} \sigma^{B}} .
$$

We now turn to the general case which includes the spin 1 linked and knotted solutions and the related spin 2 solutions. 
The general Penrose transform is given by

$$
\varphi_{A_{1}^{\prime} \cdots A_{2 h}^{\prime}}(x)=\frac{1}{2 \pi i} \oint_{\Gamma} \pi_{A_{1}^{\prime}} \cdots \pi_{A_{2 h}^{\prime}} f(Z) \pi_{B^{\prime}} d \pi^{B^{\prime}}
$$

where $\Gamma$ is a contour on the Celestial sphere of $x$ (Fig. 31) which separates the poles of $f(Z)$. The result is a spinor field which satisfies the spin- $N$ massless field equation [79]

$$
\nabla^{A A_{1}^{\prime}} \varphi_{A_{1}^{\prime} \cdots A_{2 h}^{\prime}}(x)=0 .
$$

From (306) we see that the positive helicity eigenfunctions are twistor functions of negative homogeneity. The simplest twistor function which is homogenous of degree $-2 h-2$ and has two poles is given by

$$
f(Z)=\left(\bar{A}_{\alpha} Z^{\alpha}\right)^{p}\left(\bar{B}_{\beta} Z^{\beta}\right)^{q},
$$

where $p, q<0$ and $p+q=-2 h-2$.

Consider the Penrose transform for $f(Z)$ given by

$$
f(Z)=\frac{1}{\left(\bar{A}_{\alpha} Z^{\alpha}\right)\left(\bar{B}_{\beta} Z^{\beta}\right)^{2 h+1}}
$$

which is homogeneous of degree $-2 h-2$,

$$
\begin{aligned}
\varphi_{A_{1}^{\prime} \cdots A_{2 h}^{\prime}}(x) & =\frac{1}{2 \pi i} \oint_{\Gamma} \frac{\left(o_{A_{1}}+\left(\frac{\pi_{1^{\prime}}}{\pi_{0^{\prime}}}\right) \iota_{A_{1}^{\prime}}\right) \cdots\left(o_{A_{2 h}}+\left(\frac{\pi_{1^{\prime}}}{\pi_{0^{\prime}}}\right) \iota_{A_{2 h}^{\prime}}\right)}{\left(\mathcal{A}^{0^{\prime}}+\mathcal{A}^{1^{\prime}}\left(\frac{\pi_{1^{\prime}}}{\pi_{0^{\prime}}}\right)\right)\left(\mathcal{B}^{0^{\prime}}+\mathcal{B}^{1^{\prime}}\left(\frac{\pi_{1^{\prime}}}{\pi_{0^{\prime}}}\right)\right)^{2 h+1}} d\left(\frac{\pi_{1^{\prime}}}{\pi_{0^{\prime}}}\right) \\
& =\frac{1}{2 \pi i \mathcal{A}^{1^{\prime}}\left(\mathcal{B}^{1^{\prime}}\right)^{2 h+1}} \oint_{\Gamma} \frac{\left(o_{A_{1}}+\zeta \iota_{A_{1}^{\prime}}\right) \cdots\left(o_{A_{2 h}}+\zeta \iota_{A_{2 h}^{\prime}}\right)}{(\mu+\zeta)(\nu+\zeta)^{2 h+1}} d \zeta,
\end{aligned}
$$

where $\zeta=\pi_{1^{\prime}} / \pi_{0^{\prime}}, \mu=\mathcal{A}^{0^{\prime}} / \mathcal{A}^{1^{\prime}}$, and $\nu=\mathcal{B}^{0^{\prime}} / \mathcal{B}^{1^{\prime}}$ represent again the projective coordinate and poles respectively.

Taking $\Gamma$ to enclose $-\mu$ we have

$$
\begin{aligned}
& \varphi_{A_{1}^{\prime} \cdots A_{2 h}^{\prime}}(x)=\frac{1}{\mathcal{A}^{1^{\prime}}\left(\mathcal{B}^{1^{\prime}}\right)^{2 h+1}} \operatorname{Res}_{\zeta=-\mu} \frac{\left(o_{A_{1}}+\zeta \iota_{A_{1}^{\prime}}\right) \cdots\left(o_{A_{2 h}}+\zeta \iota_{A_{2 h}^{\prime}}\right)}{(\mu+\zeta)(\nu+\zeta)^{2 h+1}} \\
& =\frac{1}{\mathcal{A}^{1^{\prime}}\left(\mathcal{B}^{1^{\prime}}\right)^{2 h+1}} \frac{\left(o_{A_{1}^{\prime}}-\mu \iota_{A_{1}^{\prime}}\right) \cdots\left(o_{A_{2 h}^{\prime}}-\mu \iota_{A_{2 h}^{\prime}}\right)}{(\nu-\mu)^{2 h+1}} \\
& =\frac{\left(\mathcal{A}^{1^{\prime}} o_{A_{1}^{\prime}}-\mathcal{A}^{0^{\prime}} \iota_{A_{1}^{\prime}}\right) \cdots\left(\mathcal{A}^{1^{\prime}} o_{A_{2 h}^{\prime}}-\mathcal{A}^{0^{\prime}} \iota_{A_{2 h}^{\prime}}\right)}{\left(\mathcal{A}^{1^{\prime}} \mathcal{B}^{0^{\prime}}-\mathcal{A}^{0^{\prime}} \mathcal{B}^{1^{\prime}}\right)^{2 h+1}} \\
& =\frac{\mathcal{A}_{A_{1}^{\prime}} \cdots \mathcal{A}_{A_{2 h}^{\prime}}}{\left(\epsilon_{A^{\prime} B^{\prime}} \mathcal{A}^{A^{\prime}} \mathcal{B}^{B^{\prime}}\right)^{2 h+1}} \\
& =2^{2 h+1} \frac{\left(i \mu_{A} x_{A_{1}^{\prime}}^{A}+\lambda_{A_{1}^{\prime}}\right) \cdots\left(i \mu_{A} x_{A_{2 h}^{\prime}}^{A}+\lambda_{A_{2 h}^{\prime}}\right)}{\left(\mu_{B} \sigma^{B}\left(x^{a}-y^{a}\right)\left(x_{a}-y_{a}\right)\right)^{2 h+1}},
\end{aligned}
$$


$\mathbf{a}$

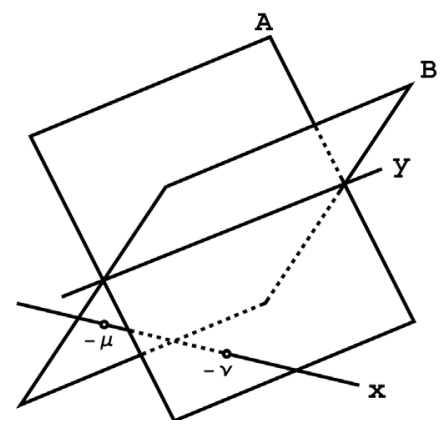

b

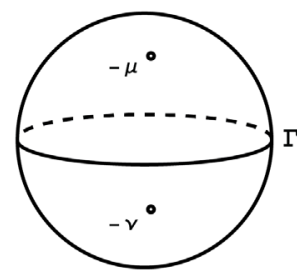

Figure 31: Geometry of the integral in the Penrose transform. a In $\mathbb{P} \mathbb{T}, A$ and $B$ are given as planes on which $f(Z)$ is singular, and they intersect at a $\mathbb{C P}^{1}$ describing a point $y \in \mathbb{C M}$. The point $x$ at which we are computing $\varphi$ is represented as a $\mathbb{C P}^{1}$ which meets $A$ and $B$ at the poles $-\mu$ and $-\nu$ of $f(Z)$. b The celestial sphere of $x$, where the contour $\Gamma$ separates the poles.

where the point $y$ is given by (318). The contour integral, whose geometry is shown in Fig. 31, thus turns the pole structure of $f(Z)$ at the point $x$ into the specific spin- $n$ massless field configuration in (323).

\section{References}

[1] E. Whittaker, A History of the Theories of Aether and Electricity, Humanities Press, New York, 1973.

[2] W. Thomson, On vortex atoms, Proc. R. Soc. Edinb. 6 (1867) 94-105.

[3] K. F. Gauss, Werke, Vol. 5, Königlichen Gessellscheft der Wissenschaften zu Göttingen, 1877, Ch. Zur Mathematischen Theorie der Electrodynamischen Wirkungen, p. 605.

[4] P. A. M. Dirac, Quantised singularities in the electromagnetic field, Proc. R. Soc. A 133 (821) (1931) 60-72. doi:10.1098/rspa.1931.0130.

[5] Y. Aharonov, D. Bohm, Significance of electromagnetic potentials in the quantum theory, Phys. Rev. 115 (1959) 485-491. doi:10.1103/PhysRev. 115.485 . 
[6] T. H. R. Skyrme, A non-linear field theory, Proc. R. Soc. A 260 (1300) (1961) 127-138. doi:10.1098/rspa.1961.0018.

[7] E. Witten, Topological quantum field theory, Comm. Math. Phys. 117 (3) (1988) 353-386. doi:10.1007/BF01223371.

[8] A. F. Rañada, A topological theory of the electromagnetic field, Lett. Math. Phys. 18 (2) (1989) 97-106. doi:10.1007/BF00401864.

[9] A. F. Rañada, Knotted solutions of the Maxwell equations in vacuum, J. Phys. A: Math. Gen. 23 (16) (1990) L815. doi:10.1088/0305-4470/23/ $16 / 007$.

[10] A. Trautman, Solutions of the Maxwell and Yang-Mills equations associated with Hopf fibrings, Int. J. Theor. Phys. 16 (8) (1977) 561-565. doi:10.1007/BF01811088.

[11] A. M. Kamchatnov, Topological solitons in magnetohydrodynamics, Sov. Phys. JETP 82 (1982) 117-124.

[12] R. Penrose, Twistor algebra, J. Math. Phys. 8 (2) (1967) 345-366. doi: 10.1063/1.1705200.

[13] R. Penrose, M. MacCallum, Twistor theory: An approach to the quantisation of fields and space-time, Phys. Rep. 6 (1972) 241-316. doi: $10.1016 / 0370-1573(73) 90008-2$.

[14] J. W. Dalhuisen, D. Bouwmeester, Twistors and electromagnetic knots, J. Phys. A: Math. Theor. 45 (2012) 135201. doi:10.1088/1751-8113/ $45 / 13 / 135201$.

[15] A. F. Rañada, J. L. Trueba, Two properties of electromagnetic knots, Phys. Lett. A 232 (1-2) (1997) 25-33. doi:10.1016/S0375-9601(97) 00366-6.

[16] H. K. Moffatt, R. L. Ricca, Helicity and the Calugareanu invariant, Proc. R. Soc. A 439 (1906) (1992) 411-429. doi:10.1098/rspa.1992.0159. 
[17] W. T. M. Irvine, D. Bouwmeester, Linked and knotted beams of light, Nature Phys. 4 (2008) 716-720. doi:10.1038/nphys1056.

[18] M. Arrayás, J. L. Trueba, A class of non-null toroidal electromagnetic fields and its relation to the model of electromagnetic knots, J. Phys. A: Math. Theor. 48 (2015) 025203. doi:10.1088/1751-8113/48/2/025203.

[19] H. Kedia, I. Bialynicki-Birula, D. Peralta-Salas, W. T. M. Irvine, Tying knots in light fields, Phys. Rev. Lett. 111 (2013) 150404. doi:10.1103/ PhysRevLett.111.150404.

[20] H. Bateman, The Mathematical Analysis of Electrical and Optical WaveMotion, Dover, New York, 1915.

[21] C. Hoyos, N. Sircar, J. Sonnenschein, New knotted solutions of Maxwell's equations, J. Phys. A: Math. Theor. 48 (2015) 255204. doi:10.1088/ $1751-8113 / 48 / 25 / 255204$.

[22] A. F. Rañada, J. L. Trueba, Ball lightning an electromagnetic knot?, Nature 383 (1996) 32. doi:10.1038/383032a0.

[23] C. B. Smiet, S. Candelaresi, A. Thompson, J. Swearngin, J. W. Dalhuisen, D. Bouwmeester, Self-organizing knotted magnetic structures in plasma, Phys. Rev. Lett. 115 (2015) 095001. doi:10.1103/PhysRevLett.115. 095001.

[24] A. Kleckner, W. T. M. Irvine, Liquid crystals: Tangled loops and knots, Nature Mat. 13 (2014) 229-231. doi:10.1038/nmat3896.

[25] Y. Kawaguchi, M. Nitta, M. Ueda, Knots in a spinor Bose-Einstein condensate, Phys. Rev. Lett. (2008) 180403doi:10.1103/PhysRevLett. 100. 180403.

[26] D. S. Hall, M. W. Ray, K. Tiurev, E. Ruokokoski, A. H. Gheorge, M. Möttönen, Tying quantum knots, Nature Phys. 12 (2016) 478-483. doi : 10.1038/nphys3624. 
[27] A. Kleckner, W. T. M. Irvine, Creation and dynamics of knotted vortices, Nature Phys. 9 (2013) 253-258. doi:10.1038/nphys2560.

[28] M. Arrayás, J. L. Trueba, Motion of charged particles in a knotted electromagnetic field, J. Phys A: Math. Theor. 43 (2010) 235401. doi: $10.1088 / 1751-8113 / 43 / 23 / 235401$.

[29] J. W. Dalhuisen, The Robinson congruence in electrodynamics and general relativity, Ph.D. thesis, Leiden University, Leiden (2014).

[30] G. A. Deschamps, Gaussian beam as a bundle of complex rays, Electronics Lett. 7 (1971) 684-685. doi:10.1049/el:19710467.

[31] A. Thompson, J. Swearngin, A. Wickes, D. Bouwmeester, Constructing a class of topological solitons in magnetohydrodynamics, Phys. Rev. E 89 (2014) 043104. doi:10.1103/PhysRevE.89.043104.

[32] A. F. Rañada, Topological electromagnetism, J. Phys. A: Math. Gen. 25 (6) (1992) 1621. doi:10.1088/0305-4470/25/6/020.

[33] A. F. Rañada, J. L. Trueba, Electromagnetic knots, Phys. Lett. A 202 (56) (1995) 337-342. doi:10.1016/0375-9601(95)00352-4.

[34] J. L. Trueba, Nudos electromagnéticos, Ph.D. thesis, Universidad Complutense, Madrid (1997).

[35] A. F. Rañada, J. L. Trueba, Topological Electromagnetism with Hidden Nonlinearity, Vol. 119, John Wiley \& Sons, Inc., New York, 2001, Ch. 2, pp. 197-253.

[36] M. Arrayás, J. L. Trueba, Exchange of helicity in a knotted electromagnetic field, Annalen der Physik 524 (2) (2012) 71-75. doi:10.1002/andp. 201100119.

[37] J. L. Trueba, A. F. Rañada, The electromagnetic helicity, Eur. J. Phys. 17 (3) (1996) 141. doi:10.1088/0143-0807/17/3/008. 
[38] A. F. Rañada, J. L. Trueba, A topological mechanism of discretization for the electric charge, Phys. Lett. B 422 (1-4) (1998) 196-200. doi: 10.1016/S0370-2693(98)00071-9.

[39] A. F. Rañada, Interplay of topology and quantization: Topological energy quantization in a cavity, Phys. Lett. A 310 (5-6) (2003) 434-444. doi: 10.1016/S0375-9601(03)00443-2.

[40] A. F. Rañada, J. L. Trueba, Topological quantization of the magnetic flux, Found. Phys. 36 (3) (2006) 427-463. doi:10.1007/s10701-005-9026-8.

[41] M. Arrayás, J. L. Trueba, A. F. Rañada, Trends in Electromagnetism - From Fundamentals to Applications, InTech, 2012, Ch. Topological Electromagnetism: Knots and Quantization Rules, pp. 71-88. doi: $10.5772 / 2108$.

${ }_{1510}^{2}$ [42] F. W. Hehl, Y. N. Obukhov, Foundations of Classical Electrodynamics: Charge, Flux and Metric, Birkhauser, Boston, 2003.

[43] C. W. Misner, K. S. Thorne, J. A. Wheeler, Gravitation, W. H. Freeman \& Co, San Francisco, 1973.

[44] J. A. Stratton, Electromagnetic Theory, McGraw-Hill, New York, 1941.

[45] S. Abdullaev, Magnetic Stochasticity in Magnetically Confined Fusion Plasmas: Chaos of Field Lines and Charged Particle Dynamics, Springer, New York, 2014. doi:10.1007/978-3-319-01890-4.

[46] B. Kosyakov, Introduction to the Classical Theory of Particles and Fields, Springer, Berlin, 2007.

[47] V. Guillemin, S. Sternberg, Symplectic Techniques in Physics, Cambridge University Press, 1984.

[48] H. Lamb, Hydrodynamics, Dover, New York, 1932. 
[49] E. A. Kuznetsov, A. V. Mikhailov, On the topological meaning of canonical Clebsch variables, Phys. Lett. A 77 (1980) 37-38. doi:10.1016/ 0375-9601 (80) 90627-1.

[50] S. J. van Enk, The covariant description of electric and magnetic field lines of null fields: Application to Hopf-Rañada solutions, J. Phys. A: Math. Theor. 46 (17) (2013) 175204. doi:10.1088/1751-8113/46/17/175204.

[51] H. K. Moffatt, The degree of knottedness of tangled vortex lines, J. Fluid Mech. 35 (1969) 117-129. doi:10.1017/S0022112069000991.

[52] W. Newcomb, Motion of magnetic lines of force, Annals of Physics 3 (1958) 347-385. doi:10.1016/0003-4916(58)90024-1.

[53] D. P. Stern, Euler potentials, Am. J. Phys. 38 (4) (1970) 494-501. doi: 10.1119/1.1976373.

${ }_{1535}$ [54] H. Hopf, Über die Abbildungen des 3-Sphäre auf die Kugelfläche, Math. Annalen 104 (1931) 637-655.

[55] R. Bott, L. W. Tu, Differential Forms in Algebraic Topology, Springer, New York, 1982.

[56] J. R. Munkres, Elements of Algebraic Topology, Addison-Wesley, New York, 1984.

[57] J. H. C. Whitehead, An expression of Hopf's invariant as an integral, Proc. Natl. Acad. Sci. USA 33 (5) (1947) 117-123. doi:10.1073/pnas. 33.5.117.

[58] F. J. Ynduráin, Mecánica Cuántica, Alianza Editorial, Madrid, 1988.

${ }_{1545}$ [59] A. F. Rañada, Fundamental Problems in Quantum Physics, Kluwer, Dordrecht, 1995, Ch. 26, pp. 267-277.

[60] G. N. Afanasiev, Y. P. Stepanovsky, The helicity of the free electromagnetic field and its physical meaning, Nuovo Cim. 109 A (1996) 271. doi:10.1007/BF02731014. 
[61] I. M. Besieris, A. M. Shaarawi, Hopf-Rañada linked and knotted light beam solution viewed as a null electromagnetic field, Opt. Lett. 34 (2009) 3887. doi:10.1364/OL.34.003887.

[62] I. Robinson, Null electromagnetic fields, J. Math. Phys. 2 (3) (1961) 290291. doi:10.1063/1.1703712.

[63] I. Bialynicki-Birula, Vortex lines of the electromagnetic field riding atop null solutions of Maxwell equations, J. Optics A: Pure Appl. Opt. 6 (2004) S181. doi : 10.1088/1464-4258/6/5/007.

[64] E. Dufraine, About homotopy classes of non-singular vector fields on the three-sphere, Qual. Theory Dyn. Syst. 3 (2002) 361-376. doi:10.1007/ BF02969412.

[65] J. Schwinger, et al., Classical Electrodynamics, Westview Press, Cambridge, 1998.

[66] L. Silberstein, Elektromagnetische grundgleichungen in bivektorieller behandlung, Annalen der Physik 327 (3) (1907) 579-586. doi:10.1002/ andp. 19073270313.

[67] H. M. Weber, Die Partiellen Differential-Gleichungen der Mathematischen Physik nach Riemann's Vorlesungen, Friedrich Vieweg und Sohn, Braunscheweig, 1901.

[68] I. Bialynicki-Birula, Z. Bialynicka-Birula, The role of the RiemannSilberstein vector in classical and quantum theories of electromagnetism, J. Phys. A: Math. Theor. 46 (2013) 053001. doi:10.1088/1751-8113/ 46/5/053001.

[69] J. Milnor, Singular Points of Complex Hypersurfaces, Princenton University Press, New Jersey, 1968.

[70] M. R. Dennis, R. P. King, B. Jack, K. O'Holleran, M. J. Padgett, Isolated optical vortex knots, Nature Phys. 6 (2010) 118. doi:10.1038/ nphys1504. 
[71] P. Appell, Quelques remarques sur la thorie des potentiels multiformes, Mathematische Annalen 34 (1887) 155.

[72] R. P. Kerr, A. Schild, Republication of: A new class of vacuum solutions of the Einstein field equations, Gen. Relativ. Gravit. 41 (2009) 2485-2499. doi:10.1007/s10714-009-0857-z.

[73] H. Bateman, The transformation of the electrodynamical equations, Proc. London Math. Soc. s2-8 (1910) 223-264. doi:10.1112/plms/s2-8.1.223.

[74] E. Cunningham, The principle of relativity in electrodynamics and an extension thereof, Proc. London Math. Soc.doi:10.1112/plms/s2-8.1. 77.

[75] V. I. Fouchtchitch, A. G. Nikitin, Symmetries of Maxwells Equations, Springer, 1987.

[76] G. E. Volovik, V. P. Mineev, Particle-like solitons in superfluid He phases, Sov. Phys. JETP 46 (1977) 401-404.

[77] I. Bialynicki-Birula, Z. Bialynicka-Birula, Gravitational waves carrying orbital angular momentum, New J. Phys. 18 (2016) 023022. doi:10. 1088/1367-2630/18/2/023022.

[78] I. Bialynicki-Birula, Z. Bialynicka-Birula, Uncertainty relation for photons, Phys. Rev. Lett. 108 (2012) 140401. doi:10.1103/PhysRevLett. 108.140401.

[79] R. Penrose, W. Rindler, Spinors and Space-Time: Volume 1, Two-Spinor Calculus and Relativistic Fields, Cambridge University Press, Cambridge, 1987.

[80] R. Penrose, W. Rindler, Spinors and Space-Time: Volume 2, Spinor and Twistor Methods in Space-Time Geometry, Cambridge University Press, Cambridge, 1988. 
[81] R. Maartens, B. A. Bassett, Gravito-electromagnetism, Class. Quant. Grav. 15 (1998) 705. doi:10.1088/0264-9381/15/3/018.

[82] D. A. Nichols, Visualizing spacetime curvature via frame-drag vortexes and tidal tendexes: General theory and weak-gravity applications, Phys. Rev. D 84 (2011) 124014. doi:10.1103/PhysRevD.84.124014.

[83] A. Thompson, J. Swearngin, A. Wickes, D. Bouwmeester, Classification of electromagnetic and gravitational Hopfions by algebraic type, J. Phys. A: Math. Theor. 48 (2015) 205202. doi:10.1088/1751-8113/48/20/205202.

[84] V. S. Semenov, D. B. Korovinski, H. K. Biernat, Euler potentials for the MHD Kamchatnov-Hopf soliton solution, Nonlinear Processes in Geophysics 9 (2002) 347-354. doi:10.5194/npg-9-347-2002.

[85] S. Candelaresi, A. Brandenburg, Topological constraints on magnetic field relaxation, in: Solar and Astrophysical Dynamos and Magnetic Activity, Proceedings of the International Astronomical Union, 2012, pp. 353-357. doi:10.1017/S1743921313002743.

[86] S. Candelaresi, F. Del Sordo, A. Brandenburg, Influence of magnetic helicity in MHD, in: Astrophysical Dynamics: From Stars to Galaxies, Vol. 6 of Proceedings of the International Astronomical Union, 2010, pp. 369-370. doi:10.1017/S1743921311017832.

[87] M. A. Berger, G. B. Field, The topological properties of magnetic helicity, J. Fluid Mech. 147 (1984) 133. doi:10.1017/S0022112084002019.

[88] M. A. Berger, Introduction to magnetic helicity, Plasma Phys. Control. Fusion 41 (12B) (1999) B167. doi:10.1088/0741-3335/41/12B/312.

[89] F. Del Sordo, S. Candelaresi, A. Brandenburg, Magnetic-field decay of three interlocked flux rings with zero linking number, Phys. Rev. E 81 (2010) 036401. doi:10.1103/PhysRevE.81.036401. 
[98] S. Chandrasekhar, E. Fermi, Problems of gravitational stability in the
presence of a magnetic field, Astrophys. J. 118 (1953) 116. doi:10.1086/
145732

[98] S. Chandrasekhar, E. Fermi, Problems of gravitational stability in the
presence of a magnetic field, Astrophys. J. 118 (1953) 116. doi:10.1086/
145732

[98] S. Chandrasekhar, E. Fermi, Problems of gravitational stability in the
presence of a magnetic field, Astrophys. J. 118 (1953) 116. doi:10.1086/
145732.

[99] D. G. Wentzel, On the shape of magnetic stars, Astrophys. J. 133 (1961)

[90] S. Candelaresi, A. Brandenburg, Decay of helical and nonhelical magnetic knots, Phys. Rev. E 84 (2011) 016406. doi:10.1103/PhysRevE. 84. 016406.

[91] S. Candelaresi, F. Del Sordo, A. Brandenburg, Decay of trefoil and other magnetic knots, in: Advances in Plasma Astrophysics, Vol. 6 of Proceedings of the International Astronomical Union, 2010, pp. 461-463. doi:10.1017/S1743921311007496.

[92] A. R. Yeates, G. Hornig, A. L. Wilmot-Smith, Topological constraints on magnetic relaxation, Phys. Rev. Lett. 105. doi:10.1103/PhysRevLett. 105.085002

[93] A. R. Yeates, G. Hornig, Dynamical constraints from field line topology in magnetic flux tubes, J. Phys A: Math. Theor. 44 (2011) 265501. doi: 10. $1088 / 1751-8113 / 44 / 26 / 265501$.

[94] R. B. White, The Theory of Toroidally Confined Plasmas, Imperial College Press, 2001.

[95] W. D'Haeseleer, W. Hitchon, J. Callen, J. Shohet, Flux Coordinates and Magnetic Field Structure, Springer-Verlag, Berlin, 1991.

[96] A. H. Boozer, Establishment of magnetic coordinates for a given magnetic field, Physics of Fluids 25 (1982) 520-521. doi:10.1063/1.863765.

[97] S. Hamada, Hydromagnetic equilibria and their proper coordinates, Nuclear Fusion 2 (1962) 23. doi:10.1088/0029-5515/2/1-2/005. 170. doi: $10.1086 / 147014$. 
[100] L. Mestel, J. Nittmann, W. P. Wood, G. A. E. Wright, The internal dynamics of the oblique rotator-II, MRAS 195 (1981) 979-1000. doi: 10.1093/mnras/195.4.979.

[101] P. D. Lasky, A. Melatos, Tilted torus magnetic fields in neutron stars and their gravitational wave signatures, Phys. Rev. D 88 (2013) 103005. doi:10.1103/PhysRevD.88.103005.

[102] P. Ghosh, F. K. Lamb, Disk accretion by magnetic neutron stars, Astrophys. J. 223 (1978) L83-L87. doi:10.1086/182734.

[103] J. D. Jackson, Classical Electrodynamics, 3rd Edition, John Wiley \& Sons, New York, 1999.

[104] S. Chandrasekhar, P. C. Kendall, On force-free magnetic fields, Astrophys. J. 126 (1957) 457. doi:10.1086/146413.

[105] H. Kedia, D. Foster, M. R. Dennis, W. T. M. Irvine, Weaving knotted vector fields with tunable helicity, arXiv:1610.02463 (2016).

${ }_{1670}$ [106] A. J. de Klerk, R. I. van der Veen, J. W. Dalhuisen, D. Bouwmeester, Cable knot vortices in electromagnetism, arXiv:1610.05285 (2016).

[107] M. Arrayás, J. L. Trueba, Collision of two hopfions, arXiv:1610.06014 (2016).

[108] A. Montesinos-Amilibia, http://www.uv.es/montesin.

1675 [109] A. F. Rañada, On the magnetic helicity, Eur. J. Phys. 13 (2) (1992) 70. doi:10.1088/0143-0807/13/2/003.

[110] D. J. Griffiths, Introduction to Electrodynamics, 3rd Edition, PrenticeHall, New Jersey, 1999.

[111] H. Flanders, Differential Forms, Academic Press, New York, 1963.

${ }_{1680}$ [112] L. A. Santaló, Vectores y Tensores, Eudeba, Buenos Aires, 1961. 
[113] M. R. Dennis, J. H. Hannay, Geometry of Calugareanu's theorem, Proc. R. Soc. A 461 (2005) 3245. doi:10.1098/rspa.2005.1527.

[114] F. B. Fuller, The writhing number of a space curve, Proc. Nat. Acad. Sci. 68 (1971) 815.

1685 [115] G. Calugareanu, L'intégrale de Gauss et l'analyse des noeuds tridimensionnels, Rev. Math. Pures Appl. 4 (1959) 5. 